\title{
Logarithmic correction for the susceptibility of the 4-dimensional weakly self-avoiding walk: a renormalisation group analysis
}

\author{
Roland Bauerschmidt*, David C. Brydges ${ }^{\dagger}$ and Gordon Slade ${ }^{\dagger}$
}

November 25, 2014

\begin{abstract}
We prove that the susceptibility of the continuous-time weakly self-avoiding walk on $\mathbb{Z}^{d}$, in the critical dimension $d=4$, has a logarithmic correction to mean-field scaling behaviour as the critical point is approached, with exponent $\frac{1}{4}$ for the logarithm. The susceptibility has been well understood previously for dimensions $d \geq 5$ using the lace expansion, but the lace expansion does not apply when $d=4$. The proof begins by rewriting the walk twopoint function as the two-point function of a supersymmetric field theory. The field theory is then analysed via a rigorous renormalisation group method developed in a companion series of papers. By providing a setting where the methods of the companion papers are applied together, the proof also serves as an example of how to assemble the various ingredients of the general renormalisation group method in a coordinated manner.
\end{abstract}

\section{Introduction and main result}

The critical behaviour of the self-avoiding walk depends on the spatial dimension $d$. The upper critical dimension is 4 , and for $d \geq 5$ the lace expansion has been used to prove that the selfavoiding walk is governed by the same critical exponents as the simple random walk [26, 39, 40, 59]. In this paper, we apply a rigorous renormalisation group analysis to study the susceptibility of the weakly self-avoiding walk in the critical dimension $d=4$.

\subsection{Continuous-time weakly self-avoiding walk}

Let $X$ be the continuous-time simple random walk on the integer lattice $\mathbb{Z}^{d}$, with $d>0$. In more detail, $X$ is the stochastic process with right-continuous sample paths that takes its steps at the times of the events of a rate- $2 d$ Poisson process. Steps are independent both of the Poisson process

\footnotetext{
* School of Mathematics, Institute for Advanced Study, Einstein Drive, Princeton, NJ 08540 USA. E-mail: roland@bauerschmidt.ca.

${ }^{\dagger}$ Department of Mathematics, University of British Columbia, Vancouver, BC, Canada V6T 1Z2. E-mail: db5d@math.ubc.ca, slade@math.ubc.ca.
} 
and of all other steps, and are taken uniformly at random to one of the $2 d$ nearest neighbours of the current position. The intersection local time up to time $T$ is defined by

$$
I(T)=\int_{0}^{T} \int_{0}^{T} \mathbb{1}_{X\left(S_{1}\right)=X\left(S_{2}\right)} d S_{1} d S_{2}=\sum_{x \in \mathbb{Z}^{d}}\left(L_{T}^{x}\right)^{2},
$$

where $L_{T}^{x}=\int_{0}^{T} \mathbb{1}_{X(S)=x} d S$ is the local time of $X$ at $x$ up to time $T$. Let $E_{a}$ denote the expectation for the process with $X(0)=a \in \mathbb{Z}^{d}$.

Given $g>0$ and $a, b \in \mathbb{Z}^{d}$, the continuous-time weakly self-avoiding walk two-point function is then defined by

$$
G_{g, \nu}(a, b)=\int_{0}^{\infty} E_{a}\left(e^{-g I(T)} \mathbb{1}_{X(T)=b}\right) e^{-\nu T} d T,
$$

where $\nu$ is a parameter (possibly negative) chosen such that the integral converges. In (1.2), selfintersections are suppressed by the factor $e^{-g I(T)}$. In the limit $g \rightarrow \infty$, if $\nu$ is simultaneously sent to $-\infty$ in a suitable $g$-dependent manner, it is known that the limit of the two-point function (1.2) is a multiple of the two-point function of the standard discrete-time strictly self-avoiding walk [16]. Our analysis is for small $g>0$; the model we study is predicted to be in the same universality class as the strictly self-avoiding walk for all $g>0$.

We set $c_{T}=c_{g, T}=E_{a}\left(e^{-g I(T)}\right)$, and define the susceptibility by

$$
\chi(g, \nu)=\sum_{b \in \mathbb{Z}^{d}} G_{g, \nu}(a, b)=\int_{0}^{\infty} c_{g, T} e^{-\nu T} d T .
$$

By translation-invariance of the simple random walk and of (1.1), $c_{T}$ and $\chi$ are independent of the point $a \in \mathbb{Z}^{d}$. In Lemma A.1, we apply a standard subadditivity argument to prove that for all dimensions $d>0$ there exists a critical value $\nu_{c}=\nu_{c}(d, g) \in(-\infty, 0]$ such that

$$
\chi(g, \nu)<\infty \text { if and only if } \nu>\nu_{c} .
$$

The rate of divergence of $\chi(g, \cdot)$ is characterised by the critical exponent $\gamma$ (assuming it exists) by

$$
\chi(g, \nu) \sim A_{g}\left(\nu-\nu_{c}\right)^{-\gamma} \text { as } \nu \downarrow \nu_{c},
$$

where $A_{g}$ and $\gamma$ are $d$-dependent constants. Throughout the paper, we write $p \sim q$ for asymptotic formulas, i.e., when $\lim p / q=1$. The exponent $\gamma$ is predicted to be universal, i.e., dependent on the dimension $d$, but otherwise independent of fine details of the model. For $d=4$, a universal logarithmic correction to this scaling has been predicted, and our main result gives a rigorous proof of this logarithmic correction. Logarithmic corrections for the scaling behaviour of the weakly selfavoiding walk in dimension 4 have been computed in the physics literature using nonrigorous renormalisation group arguments, e.g., [12, 29]. Early indications of the critical nature of the dimension $d=4$ were given in [2, 11], following proofs of triviality of the $\varphi^{4}$ field theory above dimension 4 [1,32]. For $d=4$, the weakly self-avoiding walk also coincides with the discrete-space continuous-time Edwards model (see [51, Section 10.1]).

For $d \geq 5$, it is known that (1.5) holds with critical exponent $\gamma=1$, for the weakly and strictly self-avoiding walk [26, 40]. For $d=3$, the problem remains completely unsolved from a mathematical point of view; a recent numerical estimate for $d=3$ is $\gamma \approx 1.157$ [58]. For $d=2$, it 
is predicted that $\gamma=\frac{11}{32}$ [54], and recent work suggests that the scaling behaviour can be described by $\mathrm{SLE}_{8 / 3}$ [48], but the existence neither of critical exponents nor the scaling limit has yet been proved. The case of $d=1$ is of interest for weakly self-avoiding walk, where a fairly complete understanding has been obtained [42]. For a recent survey of mathematical results about the self-avoiding walk, see [9].

\subsection{Main result}

Let $\Delta$ denote the lattice Laplacian defined by $\Delta f(x)=\sum_{e:|e|=1}(f(x+e)-f(x))$ on $\mathbb{Z}^{d}$. The lattice Green function is defined, for $m^{2}>0$, by

$$
C_{m^{2}}(x)=G_{0, m^{2}}(0, x)=\left(-\Delta+m^{2}\right)_{0 x}^{-1} .
$$

The inverse is bounded in $l^{2}\left(\mathbb{Z}^{d}\right)$-sense for $m^{2}>0$, and the limit $m^{2} \downarrow 0$ exists (pointwise in $x$ ) if $d>2$. The bubble diagram for simple random walk is the squared $\ell^{2}$ norm $B_{m^{2}}=\sum_{x \in \mathbb{Z}^{d}} C_{m^{2}}(x)^{2}$. It follows from the definition that

$$
B_{m^{2}}=\int_{0}^{\infty} \int_{0}^{\infty} P(X(T)=Y(S)) e^{-m^{2} T} e^{-m^{2} S} d T d S,
$$

where $X$ and $Y$ are two independent simple random walks starting at 0 . Hence the bubble diagram measures the expected amount of time that two independent simple random walks killed at rate $m^{2}$ intersect each other. The bubble diagram arises in an important way in our analysis, and it is convenient to define $\mathrm{B}_{m^{2}}=8 B_{m^{2}}$. By Parseval's formula and elementary calculus, as $m^{2} \downarrow 0$,

$$
\mathrm{B}_{m^{2}}=8 B_{m^{2}}=8 \int_{[-\pi, \pi]^{d}}\left|\frac{1}{4 \sum_{j=1}^{d} \sin ^{2}\left(\frac{k_{j}}{2}\right)+m^{2}}\right|^{2} \frac{d k}{(2 \pi)^{d}} \sim \begin{cases}\mathrm{b} \log m^{-2} & (d=4) \\ \mathrm{B}_{0} & (d>4),\end{cases}
$$

with $\mathrm{b}=1 /\left(2 \pi^{2}\right)$ and a $d$-dependent constant $\mathrm{B}_{0} \in(0, \infty)$. In particular, the expected time that two independent simple random walks, without killing, spend intersecting each other is finite in dimension $d>4$, but infinite in $d=4$.

Our main result is the following theorem.

Theorem 1.1. Let $d=4$ and let $g>0$ be sufficiently small. There exists $A_{g}>0$ such that, as $\varepsilon \downarrow 0$,

$$
\chi\left(g, \nu_{c}+\varepsilon\right) \sim A_{g} \varepsilon^{-1}\left(\log \varepsilon^{-1}\right)^{1 / 4} .
$$

As $g \downarrow 0$,

$$
A_{g}=(\mathrm{b} g)^{1 / 4}(1+O(g)) .
$$

Let a $=2 C_{0}(0)$ where $C_{0}(0)>0$ is the expected total time spent at the origin by the simple random walk, $C_{0}(0)=\int_{0}^{\infty} P(X(T)=0) d T=E_{0}\left(\int_{0}^{\infty} \mathbb{1}_{X(T)=0} d T\right)$. By an elementary application of Jensen's inequality, we prove in Lemma A.1 that $\nu_{c}(g) \in[-\mathrm{a} g, 0]$ for all $d>2$. As a corollary of the proof of Theorem 1.1, we obtain the following asymptotic formula for the critical value.

Theorem 1.2. Let $d=4$ and $\mathrm{a}=2 C_{0}(0)$. As $g \downarrow 0$,

$$
\nu_{c}(g)=-\mathrm{a} g+O\left(g^{2}\right) .
$$


Our method of proof of Theorems 1.1 1.2 is based on a rigorous renormalisation group analysis, and applies more widely. In particular, it is used in [5] to prove that the critical two-point function $G_{g, \nu_{c}(g)}(0, x)$ is asymptotic to a multiple of $|x|^{-2}$ as $|x| \rightarrow \infty$ in dimension $d=4$. Also, work is in progress to extend our methods to study the weakly self-avoiding walk with nearest-neighbour contact attraction [4] in dimension $d=4$. In [8], we apply the renormalisation group method to study the critical behaviour of the 4-dimensional $n$-component $|\varphi|^{4}$ spin model, for all positive integers $n \geq 1$. The existence of logarithmic corrections to scaling for certain critical 4-dimensional polymer networks, and for various critical correlation functions for the $|\varphi|^{4}$ model is proved in [60].

\subsection{Discussion}

Since $\mathrm{a}=2 \int_{0}^{\infty} P(X(T)=0) d T>0$, (1.11) shows that $\nu_{c}(g)<0$ for $g>0$. In addition, $\nu_{c}(g) \rightarrow 0$ as $g \downarrow 0$, as expected since $\nu_{c}(0)=0$ is the critical point of the simple random walk.

The factor $\varepsilon^{-1}$ in (1.9) corresponds to the linear divergence of the simple random walk susceptibility:

$$
\chi\left(0, m^{2}\right)=\sum_{x \in \mathbb{Z}^{d}} C_{m^{2}}(x)=m^{-2},
$$

while the logarithmic factor in (1.9) arises from the logarithmic divergence in (1.8). Note that $A_{g}$ tends to 0 as $g \downarrow 0$, as expected since there is no logarithmic correction for the simple random walk $(g=0)$. It has been observed that if the Fourier transform of the critical two-point function is bounded by a multiple of $|k|^{-2}$ as $k \rightarrow 0$ (as is known for $d \geq 5$ [40] and as is predicted to be true in all dimensions), then the susceptibility can have at most a logarithmic correction for $d=4$ (see [11] and [51, Theorem 1.5.4]). The exponent $\frac{1}{4}$ in (1.9) is predicted to be universal for models of self-avoiding walk in four dimensions. In particular, it is predicted to be the same for the usual discrete-time strictly self-avoiding walk [51].

For $d=4$, Theorem 1.1 and a standard Tauberian theorem [31, Chapter XIII] imply that

$$
\frac{1}{T} \int_{0}^{T} c_{S} e^{\nu_{c} S} d S \sim A_{g}(\log T)^{1 / 4} \quad(T \rightarrow \infty) .
$$

It is believed that (1.13) remains true without Cesàro average in $T$, i.e., that

$$
c_{T} \sim A_{g} e^{-\nu_{c} T}(\log T)^{1 / 4} \quad(T \rightarrow \infty),
$$

but our present estimates do not suffice to prove (1.14). Furthermore, denoting by $E_{a}^{g, T}$ the expectation for the measure of weakly self-avoiding walks of length $T$, i.e.,

$$
E_{a}^{g, T}(F(X))=\frac{E_{a}\left(e^{-g I(T)} F(X)\right)}{E_{a}\left(e^{-g I(T)}\right)}
$$

it is believed that for $p \geq 1$,

$$
\left(E_{0}^{g, T}|X(T)|^{p}\right)^{1 / p} \sim c_{g, p} T^{1 / 2}(\log T)^{1 / 8} \quad(T \rightarrow \infty)
$$

and that $\left(\lambda^{-\frac{1}{2}}(\log \lambda)^{-\frac{1}{8}} X(\lambda T)\right)_{T \geq 0}$ converges as a process to a multiple of Brownian motion as $\lambda \rightarrow$ $\infty$. (Such convergence is known for the 4-dimensional loop-erased random walk, with exponent $\frac{1}{6}$ rather than $\frac{1}{8}$ for the logarithm [46, 47].) 
Renormalisation group methods have been used previously to study weakly self-avoiding walk on a 4-dimensional hierarchical lattice. The continuous-time version of the model has been studied in the series of papers [13, 18, 19, 36]. In particular, in [19], the results of [18] were extended to prove a result analogous to (1.16) for the 4-dimensional hierarchical lattice. This was achieved by a contour integral analysis of $G_{\nu}(x)$, with $\nu$ complex. Our methods would require further development to follow the same procedure for $\mathbb{Z}^{4}$. More recently, a completely different renormalisation group approach to the discrete-time weakly self-avoiding walk on a 4-dimensional hierarchical lattice has been developed in [55]. A variant of the 4-dimensional Edwards model was analysed in [43] using a renormalisation group method; this variant is not a model of walks taking steps in a lattice, but it is presumably in the same universality class as the 4-dimensional self-avoiding walk, and the results of [43] bear some relation to our results. Some steps towards an understanding of the behaviour in dimension $d=4-\epsilon$ are taken in [53] (the work of [53] is formulated in dimension 3 but it mimics the behaviour of the nearest-neighbour model in dimension $4-\epsilon$ ).

Our renormalisation group analysis has grown out of the methods of [13, 19, but in a much extended and generalised form. It is based on an exact functional integral representation of the two-point function of the continuous-time self-avoiding walk as the two-point function of a supersymmetric quantum field theory, containing both boson and fermion fields. Such integral representations are summarised in [20]. These representations are inspired by the observation of de Gennes [35] that the self-avoiding walk problem can be regarded as the $n=0$ limit of the $n$-vector model (see also [51, Section 2.3]). The basic observation of de Gennes was that in a random walk representation of the $n$-vector model every closed loop contributes a factor $n$. When $n=0$, closed loops do not contribute, leading to self-avoiding walks. The $n$-vector model is closely related to the $n$-component $|\varphi|^{4}$ model. For $n=1$, the critical 4-dimensional $\varphi^{4}$ model was analysed using block spin renormalisation in [33,34], and via partially renormalised phase space expansion in [30]. In both approaches, the critical two-point function was controlled. Block spin methods were extended from the critical point to its neighbourhood in [38,41], where logarithmic corrections for the susceptibility and correlation length were derived for the 4-dimensional one-component $\varphi^{4}$ model (in particular, the susceptibility has exponent $\frac{1}{3}$ for the logarithm). However, it is not clear how to prove theorems about the scaling limit of the self-avoiding walk, in a rigorous mathematical sense, via an analysis of an $n \rightarrow 0$ limit of the $n$-vector model or $n$-component $|\varphi|^{4}$ theory.

On the other hand, the notion was developed in [20, 49, 50, 52, 56] that while an $n$-component boson field $\phi$ associates $n$ to each closed loop, an $n$-component fermion field $\psi$ associates $-n$. With both fields present, the net effect is to associate zero to each closed loop. This provides a way to realise de Gennes' idea, without any nonrigorous limit. Moreover, it was also understood that the fermion field can be regarded as nothing more than the differential of the boson field, with the anticommuting nature of fermions being represented by anticommuting differential forms. A representation of the self-avoiding walk two-point function as the two-point function of a supersymmetric field theory, sometimes referred to as the $\tau$-isomorphism, is central to the analysis of [13, 18, 19]. A self-contained derivation of this integral representation is given in [20], both for weakly self-avoiding walk in continuous time and for strictly self-avoiding walk in discrete time.

We use the integral representation to rewrite the two-point function of the continuous-time weakly self-avoiding walk as the two-point function of a supersymmetric field theory, and apply a rigorous renormalisation group argument to analyse the field theory. Key steps in the method are developed in the series of papers [6, 7, 14, 21 24]. In the present paper, we rely heavily on those developments and show how they can be combined to analyse the susceptibility in the 
critical dimension $d=4$. The proof here is thus not self-contained, but rather reveals its general structure, with reliance on substantial details obtained elsewhere.

In [8], we extend our analysis to the $n$-component $|\varphi|^{4}$ model. Among other results, we prove that its susceptibility obeys

$$
\chi\left(n, g, \nu_{c}+\varepsilon\right) \sim A_{g, n} \varepsilon^{-1}\left(\log \varepsilon^{-1}\right)^{(n+2) /(n+8)} .
$$

This confirms the logarithmic correction with exponent $\frac{n+2}{n+8}$ predicted for $n \geq 1$ in [12, 45, 61], and generalises the rigorous result of [38,41] for $n=1$. Setting $n=0$ in (1.17) gives a formula consistent with (1.9), but our proof for the weakly self-avoiding walk does not use any non-rigorous $n \rightarrow 0$ limit.

\subsection{Organisation}

The proof of Theorem 1.1 1.2 is divided into sections, as follows.

In Section 2, we show that the susceptibility of the weakly self-avoiding walk on $\mathbb{Z}^{d}$ is well approximated by replacing $\mathbb{Z}^{d}$ by a sequence of finite tori $\Lambda_{N}=\mathbb{Z}^{d} / L^{N} \mathbb{Z}^{d}$, as $N \rightarrow \infty$ with a fixed integer $L>1$.

In Section 3, we explain how the two-point function of weakly self-avoiding walk on the finite set $\Lambda_{N}$ can be represented as the two-point function of a supersymmetric field theory on $\Lambda_{N}$. The latter is a certain integral over differential forms on the finite-dimensional linear manifold $\mathbb{C}^{\Lambda_{N}}$, and has an interpretation as a Gaussian super-expectation. From that point onward, we no longer consider walks, and instead focus attention on the study of such integrals.

In Section 4, we express the two-point function of the weakly self-avoiding walk in terms of the two-point function of a simple random walk with renormalised parameters. We show that Theorem 1.1 follows if these renormalised parameters can be chosen suitably.

In Section 5, we explain how the integrals of Section 3 can be evaluated progressively. This progressive integration is the starting point for a multiscale analysis and defines the renormalisation group map, which we parametrise by coordinates $(V, K)$. The $V$-coordinate is 3 -dimensional and describes the important ("relevant" and "marginal") directions of the renormalisation group map. The $K$-coordinate is infinite dimensional and complements $V$ to a complete description of the problem. Thus the leading contributions in (1.9)-(1.11) will be determined by $V$, but the control of $K$ is at the heart of obtaining a mathematically rigorous result.

In Section 6, we consider the definition and properties of a single application of the renormalisation group map, by making extensive use of results developed in the companion series of papers, especially [6, 24]. This includes the definition of an infinite dimensional dynamical system which describes the infinite volume limit $\Lambda_{N} \uparrow \mathbb{Z}^{d}$.

In Section [7, we study the evolution of the renormalisation group map, by applying a result of [7] concerning dynamical systems, together with the estimates given in Section 6. We analyse the stability of the renormalisation group map near the Gaussian fixed point corresponding to the simple random walk $g=0$. More precisely, we construct a centre stable manifold near this fixed point.

In Section 8, we prove Theorems $1.1,1.2$ using the results of Sections 207. The centre stable manifold constructed in Section 7 plays a crucial role in the identification of the critical point. To deduce the logarithmic correction for the susceptibility for $d=4$, we study infinitesimal deviations 
of the flow from the centre stable manifold, which allows the derivative of $\chi$ with respect to $\nu$ to be computed. An elementary but important technical aspect is that the results of the previous sections permit us to focus our attention on this derivative for the case of finite volume $\Lambda_{N}$.

Throughout the remainder of this paper, we emphasise the source of the power of the logarithm in (1.9) by writing

$$
\gamma=\frac{1}{4}
$$

Thus $\gamma$ does not denote the critical exponent as in (1.5), but rather the exponent of the logarithmic correction to the critical exponent 1.

\section{Finite volume approximation}

The proof begins by approximation of $\mathbb{Z}^{d}$ by a sequence of finite tori of period $L^{N}$, which we denote by $\Lambda=\Lambda_{N}=\mathbb{Z}^{d} / L^{N} \mathbb{Z}^{d}$. Let $E_{a}^{\Lambda_{N}}$ denote the expectation of the continuous-time simple random walk on $\Lambda_{N}$, started from $a \in \Lambda_{N}$. Let

$$
c_{N, T}(a, b)=E_{a}^{\Lambda_{N}}\left(e^{-g I(T)} \mathbb{1}_{X(T)=b}\right), \quad c_{N, T}=E_{a}^{\Lambda_{N}}\left(e^{-g I(T)}\right) .
$$

We define the two-point function for the torus by

$$
G_{N, \nu}(a, b)=\int_{0}^{\infty} c_{N, T}(a, b) e^{-\nu T} d T,
$$

and define the corresponding torus susceptibility by

$$
\chi_{N}(\nu)=\sum_{b \in \Lambda_{N}} G_{N, \nu}(a, b)=\int_{0}^{\infty} c_{N, T} e^{-\nu T} d T .
$$

By the Cauchy-Schwarz inequality, $T=\sum_{x \in \Lambda} L_{T}^{x} \leq(|\Lambda| I(T))^{1 / 2}$, and hence

$$
\chi_{N}(\nu) \leq \int_{0}^{\infty} e^{-g T^{2} /\left|\Lambda_{N}\right|} e^{-\nu T} d T<\infty \quad \text { for all } \nu \in \mathbb{R}
$$

The following lemma shows that $\chi_{N}$ is a good approximation to $\chi$.

Lemma 2.1. Let $d>0$. For all $\nu \in \mathbb{R}, \chi_{N}(\nu)$ is non-decreasing in $N$, and obeys $\lim _{N \rightarrow \infty} \chi_{N}(\nu)=$ $\chi(\nu)$ (with $\chi(\nu)=\infty$ for $\left.\nu \leq \nu_{c}\right)$. The functions $\chi_{N}$ and $\chi$ are analytic on $\left\{\nu \in \mathbb{C}: \operatorname{Re} \nu>\nu_{c}\right\}$, and $\chi_{N}$ and all its derivatives converge uniformly on compact subsets of $\operatorname{Re} \nu>\nu_{c}$ to $\chi$ and its derivatives.

Proof. Let $c_{N, T}=E^{\Lambda_{N}}\left(e^{-g I(T)}\right)$ as in (2.1), and let $c_{T}=E\left(e^{-g I(T)}\right)$ as in (1.3). We first show that

$$
c_{N, T} \leq c_{N+1, T} \leq c_{T}, \quad \lim _{N \rightarrow \infty} c_{N, T}=c_{T}
$$

(for the inequality we assume $L^{N} \geq 3$ ).

To see this, we observe that there is a one-to-one correspondence between nearest-neighbour walks on $\mathbb{Z}^{d}$ started at the origin and such walks on the finite torus $\mathbb{Z}_{n}^{d}$ if $n \geq 3$, by folding a walk 
on $\mathbb{Z}^{d}$ (the image under the canonical projection $\mathbb{Z}^{d} \rightarrow \mathbb{Z}_{n}^{d}$ ), and corresponding unfolding of walks on $\mathbb{Z}_{n}^{d}$ (unique for the nearest-neighbour walk when $n \geq 3$ ). Given a walk $X$ on $\mathbb{Z}^{d}$ starting at 0 , we denote the folded walk on $\mathbb{Z}_{n}^{d}$ by $X^{n}$. This allows us to couple walks on tori of different diameter via the distribution of walks on $\mathbb{Z}^{d}$. We denote the local time of a walk $X$ up to time $T$ by $L_{T}^{x}(X)=\int_{0}^{T} \mathbb{1}_{X(S)=x} d S$, and similarly the intersection local time by $I_{T}(X)$. Given $X$ and a positive integer $k$, we obtain

$$
\begin{aligned}
I_{T}\left(X^{k n}\right) & =\sum_{x \in \mathbb{Z}_{k n}^{d}}\left(L_{T}^{x}\left(X^{k n}\right)\right)^{2}=\sum_{x \in \mathbb{Z}_{n}^{d}} \sum_{y \in \mathbb{Z}^{d}:\|y\|_{\infty}<k}\left(L_{T}^{x+y n}\left(X^{k n}\right)\right)^{2} \\
& \leq \sum_{x \in \mathbb{Z}_{n}^{d}}\left(\sum_{y \in \mathbb{Z}^{d}:\|y\|_{\infty}<k} L_{T}^{x+y n}\left(X^{k n}\right)\right)^{2}=\sum_{x \in \mathbb{Z}_{n}^{d}}\left(L_{T}^{x}\left(X^{n}\right)\right)^{2}=I_{T}\left(X^{n}\right) .
\end{aligned}
$$

Thus, with $n=L^{N}, k=L$, and with $X$ fixed,

$$
e^{-g I_{N+1, T}(X)} \geq e^{-g I_{N, T}(X)} .
$$

Now we take the expectation over $X$ to obtain the first inequality of (2.5). This shows monotonicity in $N$ of $c_{N, T}$. Also, a folded walk can only have more intersections than its unfolding, so $I_{T}\left(X^{n}\right) \geq$ $I_{T}(X)$ for any walk $X$ on $\mathbb{Z}^{d}$ and for any $n$, so we also have $c_{N, T} \leq c_{N+1, T} \leq c_{T}$.

For the convergence of $c_{T, N}$ to $c_{T}$, we first note that walks which do not reach distance $\frac{1}{2} L^{N}$ from the origin do not contribute to the difference. Walks which do must take at least $\frac{1}{2} L^{N}$ steps. Therefore, since $I(T) \geq 0$, we conclude that $\left|c_{T}-c_{T, N}\right| \leq 2 \mathbb{P}\left(M_{T}>\frac{1}{2} L^{N}\right)$, where $M_{T}$ is a rate-2d Poisson process. The probability in the upper bound goes to zero as $N \rightarrow \infty, \operatorname{so}_{N \rightarrow \infty} \lim _{N, T}=c_{T}$, and (2.5) is proved.

With the monotone convergence theorem, (2.5) gives

$$
\chi(\nu)=\int_{0}^{\infty} \lim _{N \rightarrow \infty} c_{N, T} e^{-\nu T} d T=\lim _{N \rightarrow \infty} \chi_{N}(\nu) \quad \text { for } \nu \in \mathbb{R}
$$

where both sides are finite if and only if $\nu>\nu_{c}$. Also, since $\left|c_{N, T} e^{-\nu T}\right| \leq c_{N, T} e^{-(\operatorname{Re} \nu) T} \leq c_{T} e^{-(\operatorname{Re} \nu) T}$, it follows from the dominated convergence theorem that

$$
\chi(\nu)=\lim _{N \rightarrow \infty} \chi_{N}(\nu) \text { for } \operatorname{Re} \nu>\nu_{c} .
$$

The analyticity of $\chi$ and $\chi_{N}$ follows from analyticity of Laplace transforms, and the desired compact convergence of $\chi_{N}$ and all its derivatives then follows from Montel's theorem.

\section{Integral representation}

The next step in the proof is to represent the two-point function for walks on the finite set $\Lambda$ by an integral over the finite dimensional space $\mathbb{C}^{\Lambda}$. Before entering into the details of the representation, we first briefly recall some basic facts about integration of differential forms. 


\subsection{Integration of differential forms}

Let $\Lambda=\{1, \ldots, M\}$ be a finite set (e.g., a discrete torus) of cardinality $M$. Let $u_{1}, v_{1}, \ldots, u_{M}, v_{M}$ be standard coordinates on $\mathbb{R}^{2 M}$. Then $d u_{1} \wedge d v_{1} \wedge \cdots \wedge d u_{M} \wedge d v_{M}$ is the standard volume form on $\mathbb{R}^{2 M}$, where $\wedge$ denotes the usual anticommuting wedge product (see [57, Chapter 10] for an introduction). In the following, we drop the wedge from the notation and write simply $d u_{i} d v_{j}$ in place of $d u_{i} \wedge d v_{j}$. The one-forms $d u_{i}, d v_{j}$ generate the Grassmann algebra of differential forms on $\mathbb{R}^{2 M}$. A form which is a function of $u, v$ times a product of $p$ differentials is said to have degree $p$, for $p \geq 0$. A sum of differential forms of even degree is called even. We will use the term form as an abbreviation for "differential form."

Any form $F$ of degree $2 M$ can be written as $F=f(u, v) d u_{1} d v_{1} \cdots d u_{M} d v_{M}$, and its integral is defined by

$$
\int F=\int_{\mathbb{R}^{2 M}} f(u, v) d u_{1} d v_{1} \cdots d u_{M} d v_{M},
$$

where the right-hand side is the usual Lebesgue integral of $f$ over $\mathbb{R}^{2 M}$. For $p \neq 2 M$, we define the integral over $\mathbb{R}^{2 M}$ of a form of degree $p$ to be zero. Then the integral of an arbitrary form, which is a linear combination of forms of degree $p$ for different values of $p$, is defined by linearity; only its component of top degree affects the value of the integral.

We introduce complex coordinates by setting $\phi_{x}=u_{x}+i v_{x}, \bar{\phi}_{x}=u_{x}-i v_{x}$ and $d \phi_{x}=d u_{x}+i d v_{x}$, $d \bar{\phi}_{x}=d u_{x}-i d v_{x}$, for $x \in \Lambda$. Since the wedge product is anticommutative, the following pairs all anticommute for every $x, y \in \Lambda: d \phi_{x}$ and $d \phi_{y}, d \bar{\phi}_{x}$ and $d \phi_{y}, d \bar{\phi}_{x}$ and $d \bar{\phi}_{y}$. In addition,

$$
d \bar{\phi}_{x} d \phi_{x}=2 i d u_{x} d v_{x} .
$$

The integral of a function $f(\phi, \bar{\phi})$ (i.e., a 0 -form) with respect to $\prod_{x \in \Lambda} d \bar{\phi}_{x} d \phi_{x}$ is thus given by $(2 i)^{M}$ times the integral of $f(u+i v, u-i v)$ over $\mathbb{R}^{2 M}$. Note that the product here can be taken in any order, since each factor $d \bar{\phi}_{x} d \phi_{x}$ has even degree (namely degree two). It is convenient for us to define

$$
\psi_{x}=\frac{1}{\sqrt{2 \pi i}} d \phi_{x}, \quad \bar{\psi}_{x}=\frac{1}{\sqrt{2 \pi i}} d \bar{\phi}_{x},
$$

where we fix a choice of the square root and use this choice henceforth. Then

$$
\bar{\psi}_{x} \psi_{x}=\frac{1}{2 \pi i} d \bar{\phi}_{x} d \phi_{x}=\frac{1}{\pi} d u_{x} d v_{x} .
$$

By definition, a differential form $F$ can be written as

$$
\sum_{x, y} F_{x, y}(\phi, \bar{\phi}) \psi^{x} \bar{\psi}^{y},
$$

where the sum is over sequences $x$ and $y$ in $\Lambda$ (of any length) and $\psi^{x}=\psi_{x_{1}} \cdots \psi_{x_{p}}$ for $x=$ $\left(x_{1}, \ldots, x_{p}\right) \in \Lambda^{p}$. Given a subset $X \subset \Lambda$, we denote by $\mathcal{N}(X)$ the algebra of even forms $F$ with the restriction that the sum in (3.5) extends only over sequences in $X$ and the coefficients $F_{x, y}$ depend only on $\left(\phi_{x}, \bar{\phi}_{x}\right)_{x \in X}$. The algebra $\mathcal{N}(X)$ is commutative and associative. For the special case $X=\Lambda$, we write simply $\mathcal{N}=\mathcal{N}(X)$. Later we discuss norms on $\mathcal{N}$ that impose regularity conditions on its elements. 


\subsection{Functions of forms}

We refer to the variables $\left(\phi_{x}\right)$ and the forms $\left(\psi_{x}\right)$ in (3.5) as boson and fermion fields, respectively. We view $\psi$ as an anticommuting analogue of $\phi$ and think of a differential form as a function of $\phi$ and $\psi$. Differential forms have degree at most $2 M$ and therefore, as "functions" of $\psi$, they are polynomial with degree at most $2 M$. We use terminology corresponding to this view of differential forms. For example, by setting $\bar{\psi}=\psi=0$ in a form we mean to take the projection to its degree-0 part. In our context, the forms $\psi, \bar{\psi}$ are often called Grassmann variables, and integrals of "functions" of $\psi$ in the standard sense of differential forms, as explained in Section 3.1, are the same as the Berezin integral [10,49].

We also define functions of even forms. Let $F=\left(F_{j}\right)_{j \in J}$ be a finite collection of even forms. We say that $F$ is even. Let $F_{j}^{0}$ denote the degree-0 part of $F_{j}$. Given a $C^{\infty}$ function $f: \mathbb{R}^{J} \rightarrow \mathbb{C}$ we define a form $f(F)$ by its Taylor series about the degree- 0 part of $F$, i.e.,

$$
f(F)=\sum_{\alpha} \frac{1}{\alpha !} f^{(\alpha)}\left(F^{0}\right)\left(F-F^{0}\right)^{\alpha}
$$

where $\alpha=\left(\alpha_{j}\right)_{j \in J}$ is a multi-index, with $\alpha !=\prod_{j \in J} \alpha_{j}$ ! , and $\left(F-F^{0}\right)^{\alpha}=\prod_{j \in J}\left(F_{j}-F_{j}^{0}\right)^{\alpha_{j}}$. Note that the summation terminates as soon as $\sum_{j \in J} \alpha_{j}=M$ since higher order forms vanish, and that the order of the product on the right-hand side is immaterial when $F$ is even. For example,

$$
e^{-\phi_{x} \bar{\phi}_{x}-\psi_{x} \bar{\psi}_{x}}=e^{-\phi_{x} \bar{\phi}_{x}}\left(1-\psi_{x} \bar{\psi}_{x}\right) \text {. }
$$

\subsection{Identity for two-point function}

Let $\Lambda=\Lambda_{N}$ now denote the discrete torus in $\mathbb{Z}^{d}$, as in Section 2. For $x \in \Lambda$, we define the forms

$$
\tau_{x}=\phi_{x} \bar{\phi}_{x}+\psi_{x} \wedge \bar{\psi}_{x}
$$

and

$$
\tau_{\Delta, x}=\frac{1}{2}\left(\phi_{x}(-\Delta \bar{\phi})_{x}+(-\Delta \phi)_{x} \bar{\phi}_{x}+\psi_{x} \wedge(-\Delta \bar{\psi})_{x}+(-\Delta \psi)_{x} \wedge \bar{\psi}_{x}\right),
$$

where $\Delta$ is the lattice Laplacian on $\Lambda$ given by $\Delta \phi_{x}=\sum_{e:|e|=1}\left(\phi_{x+e}-\phi_{x}\right)$. Atypically, we have left the wedge product $\wedge$ explicit in the above definitions, for emphasis. The following proposition is proved in [13, 19]; see [20, Theorem 5.1] for a self-contained proof. The integrand and integral on the right-hand side of (3.10) are as defined in Section 3.1.

Proposition 3.1. Let $d>0$. For $g>0$ and $\nu \in \mathbb{R}$, or $g=0$ and $\nu>0$,

$$
G_{N, g, \nu}(a, b)=\int e^{-\sum_{x \in \Lambda}\left(\tau_{\Delta, x}+g \tau_{x}^{2}+\nu \tau_{x}\right)} \bar{\phi}_{a} \phi_{b} .
$$

Note that, by summation by parts on the torus $\Lambda$,

$$
\sum_{x \in \Lambda} \tau_{\Delta, x}=\sum_{x \in \Lambda}\left(\phi_{x}(-\Delta \bar{\phi})_{x}+\psi_{x} \wedge(-\Delta \bar{\psi})_{x}\right),
$$

so the symmetrisation in (3.9) is not needed for (3.10) (it is absent also in [20, Theorem 5.1]). However, later we need sums $\sum_{x \in X} \tau_{\Delta, x}$ with $X \subset \Lambda$ a proper subset, and there symmetrisation is important. 
The right-hand side of (3.10) is the two-point function of a supersymmetric field theory with quartic self-interaction. The partition function that results by dropping the factor $\bar{\phi}_{a} \phi_{b}$ on the right-hand side of (3.10) turns out to be simply equal to 1. More generally, there is the remarkable self-normalisation property that

$$
\int e^{-\sum_{x \in \Lambda}\left(p_{x} \tau_{\Delta, x}+q_{x} \tau_{x}^{2}+r_{x} \tau_{x}\right)}=1 \quad \text { for all } p_{x} \geq 0, q_{x}>0, r_{x} \in \mathbb{R}
$$

as a result of supersymmetry. Thus supersymmetry provides, in particular, the simplification that there is no need to conduct any analysis of a partition function. A self-contained proof of (3.12) and generalisations, as well as a brief discussion of supersymmetry, can be found in [20].

In the remainder of this section, we discuss some of the intuition associated to (3.10), but we do not make use of it afterwards. Since $\tau^{2}=|\phi|^{4}+2|\phi|^{2}|\psi|^{2}$, the fermionic part of the right-hand side of (3.10) is

$$
e^{-\sum_{x, y \in \Lambda} \psi_{x} A_{x y} \bar{\psi}_{y}} \quad \text { with } A_{x y}=-\Delta_{x y}+\left(\nu+2 g \phi_{x} \bar{\phi}_{x}\right) \delta_{x y}
$$

One way to evaluate the integral on the right-hand side of (3.10) is to expand the exponential (3.13), keep only the top-degree part, and rearrange the order of the differentials to obtain the standard volume form. The anti-commutativity produces a determinant, with the result that the top-degree part of $e^{-\sum_{x \in \Lambda}\left(\tau_{\Delta, x}+g \tau_{x}^{2}+\nu \tau_{x}\right)}$ is equal to

$$
\operatorname{det}\left(-\Delta+\nu+2 g|\phi|^{2}\right) e^{-\sum_{x \in \Lambda}\left(\phi_{x}(-\Delta \bar{\phi})_{x}+g\left|\phi_{x}\right|^{4}+\nu\left|\phi_{x}\right|^{2}\right)} \prod_{x \in \Lambda} \frac{d \bar{\phi}_{x} d \phi_{x}}{2 \pi i} .
$$

Thus there is an alternate version of the identity (3.10), in which the exponential on its right-hand side is replaced by (3.14). This procedure of integrating out of fermions has been useful in the study of other supersymmetric models [27,28], but we do not follow this approach. Instead, we work directly with the right-hand side of (3.10) throughout the entire analysis.

If we drop the $\psi$ terms in the definitions of $\tau, \tau_{\Delta}$ in (3.8)-(3.9) (or equivalently drop the determinant in (3.14) $)$, consider a real-valued boson field $\varphi_{x}$ rather than a complex-valued one, and integrate with respect to the Lebesgue measure $\prod_{x \in \Lambda} d \varphi_{x}$ on $\mathbb{R}^{\Lambda}$, the right-hand side of (3.10) becomes the integral

$$
\int_{\mathbb{R}^{\Lambda}} \varphi_{a} \varphi_{b} e^{-\sum_{x \in \Lambda}\left(\frac{1}{2} \varphi_{x}(-\Delta \varphi)_{x}+\frac{1}{4} g \varphi_{x}^{4}+\frac{1}{2} \nu \varphi_{x}^{2}\right)} \prod_{x \in \Lambda} d \varphi_{x}
$$

which is the unnormalised two-point function of the $\varphi^{4}$-model. The $\varphi^{4}$-model is a spin system in which each point $x \in \Lambda$ carries a spin variable $\varphi_{x} \in \mathbb{R}$. It is a perturbation of the massless Gaussian free field, in which each spin has a factor $e^{-U\left(\varphi_{x}\right)}$ with $U(t)=\frac{1}{4} g t^{4}+\frac{1}{2} \nu t^{2}$. The term $\sum_{x \in \Lambda} \frac{1}{2} \varphi_{x}(-\Delta \varphi)_{x}$ in the exponent can also be written as a sum of squares of the gradient of $\varphi_{x}$, so fields with small gradient receive larger weight, which is a ferromagnetic interaction. Completing the square gives

$$
U(t)=\frac{1}{4} g\left(t^{2}+\frac{\nu}{g}\right)^{2}-\frac{\nu^{2}}{4 g}
$$

The term $-\nu^{2} / 4 g$ on the right-hand side cancels upon normalisation of the integral (3.15) and is unimportant. As in Theorem 1.2, we are interested in negative values of $\nu$, so that $U(t)$ has a double well shape, with minima at $t= \pm \sqrt{|\nu| / g}$ separated by a barrier of height $\nu^{2} / g$. As $\nu$ 
becomes increasingly negative, the two wells become increasingly deep and increasingly separated, so it is plausible that there is a critical value of $\nu$ at which long range correlations develop due to the values of $\varphi_{x}$ becoming concentrated at the minima of $U$. The behaviour in dimension $d=4$ at and near this critical point is the subject of the renormalisation group analyses of [30, 33, 34, 38, 41]. Although our method of proof is not based on the above picture, the picture provide some intuition. Our method is applied to the $n$-component $|\varphi|^{4}$ model in [8,60].

\subsection{Gaussian super-expectation}

The right-hand side of (3.10) is an instance of a Gaussian super-expectation, defined as follows. First, given a $\Lambda \times \Lambda$ matrix $A$, let

$$
S_{A}(\Lambda)=\sum_{x, y \in \Lambda}\left(\phi_{x} A_{x y} \bar{\phi}_{y}+\psi_{x} A_{x y} \bar{\psi}_{y}\right) .
$$

Definition 3.2. Given a positive-definite $\Lambda \times \Lambda$ matrix $C$, let $A=C^{-1}$. Given a form $F$ on $\mathbb{C}^{\Lambda}$, we define the Gaussian super-expectation of $F$, with covariance $C$, by

$$
\mathbb{E}_{C} F=\int e^{-S_{A}(\Lambda)} F
$$

where the integral on the right-hand side is defined as in Section 3.1 .

In the special case that $f$ is a 0 -form, or in other words $f$ is a function of $(\phi, \bar{\phi}), \mathbb{E}_{C} f$ is equal to the standard Gaussian expectation for a complex-valued random field $\phi$ with covariance $C$ (see [20, Proposition 4.1]), i.e.,

$$
\mathbb{E}_{C} f=\int_{\mathbb{C}^{\Lambda}} f(\phi, \bar{\phi}) d \mu_{C}(\phi, \bar{\phi}), \quad d \mu_{C}(\phi, \bar{\phi})=Z_{C}^{-1} e^{-\sum_{x, y \in \Lambda} \phi_{x} C_{x y}^{-1} \bar{\phi}_{y}} \prod_{x \in \Lambda} d \bar{\phi}_{x} d \phi_{x}
$$

where $Z_{C}$ is a normalisation constant such that $\mathbb{E}_{C} 1=1$. In particular, $\mathbb{E}_{C} \bar{\phi}_{a} \phi_{b}=C_{a b}$, i.e., $C$ is the covariance of the Gaussian measure $\mu_{C}$. For a general differential form, $\mathbb{E}_{C} F$ loses the probabilistic interpretation, but nevertheless many of the important properties of Gaussian integrals continue to hold for the Gaussian super-expectation. For example, $C$ is also the "covariance" of $\psi$, i.e., $\mathbb{E}_{C} \bar{\psi}_{a} \psi_{b}=-\mathbb{E}_{C} \psi_{b} \bar{\psi}_{a}=C_{a b}$. Also, as a result of supersymmetry and in contrast to the usual Gaussian measure $\mu_{C}$ in (3.19), there is no normalisation constant in (3.18). Further discussion of the Gaussian super-expectation is given in Section 5.1 below.

Formally, the right-hand side of (3.10) looks like the Gaussian super-expectation of $e^{-\sum_{x \in \Lambda} g \tau_{x}^{2}}$ with $A=-\Delta+\nu$ where $\Delta$ is the discrete Laplace operator on $\Lambda_{N}$. However, the critical point $\nu_{c}$ is negative according to (1.11). Thus $-\Delta+\nu$ is an indefinite matrix for the values of $\nu$ of interest, and therefore is not a proper covariance of a Gaussian expectation. On the other hand, for any $\epsilon>0,(3.10)$ can be written as

$$
\int e^{-\sum_{x \in \Lambda}\left(\tau_{\Delta, x}+g \tau_{x}^{2}+\nu \tau_{x}\right)} \bar{\phi}_{a} \phi_{b}=\mathbb{E}_{C}\left(e^{-\sum_{x \in \Lambda}\left(g \tau_{x}^{2}+(\nu-\epsilon) \tau_{x}\right)} \bar{\phi}_{a} \phi_{b}\right)
$$

with the positive-definite covariance $C=(-\Delta+\epsilon)^{-1}$. A careful division of the right-hand side of (3.10) into a Gaussian expectation and a perturbation is central to our analysis and is discussed next, where we divide not just the $\tau$ term but also the $\tau_{\Delta}$ term. 


\section{Reformulation in renormalised parameters}

\subsection{Approximation by simple random walk}

An important notion in theoretical physics is that it is often possible to approximate an interacting system by an effective non-interacting system with renormalised parameters. As a first step towards implementing this, we write temporarily

$$
V_{g, \nu, z ; x}=V_{g, \nu, z ; x}(\phi, \psi)=g \tau_{x}^{2}+\nu \tau_{x}+z \tau_{\Delta, x} .
$$

We fix $m^{2}>0$ and $z_{0}>-1$, and set $\left(\phi^{\prime}, \psi^{\prime}\right)=\left(\left(1+z_{0}\right)^{1 / 2} \phi,\left(1+z_{0}\right)^{1 / 2} \psi\right)$ and similarly for the conjugates. By definition,

$$
V_{g, \nu, 1 ; x}\left(\phi^{\prime}, \psi^{\prime}\right)=V_{0, m^{2}, 1 ; x}(\phi, \psi)+V_{g_{0}, \nu_{0}, z_{0} ; x}(\phi, \psi),
$$

where

$$
g_{0}=g\left(1+z_{0}\right)^{2}, \quad \nu_{0}=\left(1+z_{0}\right) \nu-m^{2},
$$

or equivalently,

$$
g=\frac{g_{0}}{\left(1+z_{0}\right)^{2}}, \quad \nu=\frac{\nu_{0}+m^{2}}{1+z_{0}}
$$

For $X \subset \Lambda$, we define

$$
V_{0}(X)=\sum_{x \in X} V_{g_{0}, \nu_{0}, z_{0} ; x}=\sum_{x \in X}\left(g_{0} \tau_{x}^{2}+\nu_{0} \tau_{x}+z_{0} \tau_{\Delta, x}\right)
$$

Let $C=\left(-\Delta+m^{2}\right)^{-1}$, with $\Delta$ the discrete Laplacian on $\Lambda_{N}$. By making the change of variables $\phi_{x} \mapsto \phi^{\prime}=\left(1+z_{0}\right)^{1 / 2} \phi_{x}$, and writing $F^{\prime}(\phi, \psi)=F\left(\phi^{\prime}, \psi^{\prime}\right)$, we obtain

$$
\int F e^{-\sum_{x \in \Lambda}\left(\tau_{\Delta, x}+g \tau_{x}^{2}+\nu \tau_{x}\right)}=\mathbb{E}_{C} F^{\prime} e^{-V_{0}(\Lambda)}
$$

There is no explicit Jacobian factor, since we also make the change of variables in the differentials $\psi, \bar{\psi}$. Then, by (4.6),

$$
G_{N}(g, \nu)=\left(1+z_{0}\right) \hat{G}_{N}\left(m^{2}, g_{0}, \nu_{0}, z_{0}\right)
$$

where

$$
\hat{G}_{N}\left(m^{2}, g_{0}, \nu_{0}, z_{0}\right)=\mathbb{E}_{C}\left(e^{-V_{0}(\Lambda)} \bar{\phi}_{a} \phi_{b}\right)
$$

(for $g_{0}>0$ the right-hand side is convergent for all $\nu_{0} \in \mathbb{R}$ and $m^{2}>0$ ). In (4.7), we write the twopoint function as $G_{N}(g, \nu)$ instead of $G_{N, g, \nu}(a, b)$, with $a, b$ now suppressed, since the lattice points $a, b$ do not play a primary role for the moment and it is rather the dependence on $g, \nu$ that we wish to emphasise. If we set $V_{0}=0$ on the right-hand side of (4.7), the result is a multiple of the simple random walk two-point function with mass $m^{2}$. We regard the factor $e^{-V_{0}}$ as a perturbation of the simple random walk. Much of our effort lies in the choice of the renormalised parameters $\left(m^{2}, z_{0}\right)$ and the determination of their relation to the original (or "bare," in terminology of quantum field theory) parameters $(g, \nu)$, so that the perturbation is small and the approximation by simple random walk is a good one. 
We define an analogous quantity for the susceptibility by

$$
\hat{\chi}_{N}\left(m^{2}, g_{0}, \nu_{0}, z_{0}\right)=\sum_{x \in \Lambda} \mathbb{E}_{C}\left(e^{-V_{0}(\Lambda)} \bar{\phi}_{0} \phi_{x}\right) .
$$

Then, as in (4.7), $\chi$ and $\hat{\chi}$ are related by

$$
\chi_{N}(g, \nu)=\left(1+z_{0}\right) \hat{\chi}_{N}\left(m^{2}, g_{0}, \nu_{0}, z_{0}\right) .
$$

The limit

$$
\hat{\chi}\left(m^{2}, g_{0}, \nu_{0}, z_{0}\right)=\lim _{N \rightarrow \infty} \hat{\chi}_{N}\left(m^{2}, g_{0}, \nu_{0}, z_{0}\right)
$$

exists by Lemma 2.1 and (4.10), and

$$
\chi(g, \nu)=\left(1+z_{0}\right) \hat{\chi}\left(m^{2}, g_{0}, \nu_{0}, z_{0}\right) .
$$

Also, for $\nu>\nu_{c}$, it follows from Lemma 2.1 and the chain rule that

$$
\frac{\partial \chi}{\partial \nu}(g, \nu)=\left(1+z_{0}\right)^{2} \frac{\partial \hat{\chi}}{\partial \nu_{0}}\left(m^{2}, g_{0}, \nu_{0}, z_{0}\right)=\left(1+z_{0}\right)^{2} \lim _{N \rightarrow \infty} \frac{\partial \hat{\chi}_{N}}{\partial \nu_{0}}\left(m^{2}, g_{0}, \nu_{0}, z_{0}\right)
$$

The finite volume susceptibility $\hat{\chi}_{N}$ can be conveniently re-expressed in terms of a generating functional, as follows. Given an external field (or test function) $J: \Lambda \rightarrow \mathbb{C}$, we write

$$
(J, \bar{\phi})=\sum_{x \in \Lambda} J_{x} \bar{\phi}_{x}, \quad(\bar{J}, \phi)=\sum_{x \in \Lambda} \bar{J}_{x} \phi_{x}
$$

Let 1 denote the constant test function $1_{x}=1$ for all $x \in \Lambda$. Let $m^{2}>0, g_{0}>0, \nu_{0} \in \mathbb{R}, z_{0}>-1$. By (4.9) and translation invariance,

$$
\hat{\chi}_{N}=\hat{\chi}_{N}\left(m^{2}, g_{0}, \nu_{0}, z_{0}\right)=|\Lambda|^{-1} \mathbb{E}_{C}\left((1, \bar{\phi})(1, \phi) Z_{0}\right),
$$

where $C=\left(-\Delta+m^{2}\right)^{-1}$ and $Z_{0}=Z_{0}\left(g_{0}, \nu_{0}, z_{0}\right)=e^{-V_{0}(\Lambda)}$. We define the bosonic generating functional $\Sigma: \mathbb{C}^{\Lambda} \rightarrow \mathbb{C}$ by

$$
\Sigma(J, \bar{J})=\mathbb{E}_{C}\left(e^{(J, \bar{\phi})+(\phi, \bar{J})} Z_{0}\right)
$$

Then

$$
\hat{\chi}_{N}=|\Lambda|^{-1} D^{2} \Sigma(0,0 ;(0,1),(1,0))=|\Lambda|^{-1} D^{2} \Sigma(0,0 ; 1,1),
$$

where the right-hand sides involve the directional derivative with directions equal to the constant function 1 in the first and second argument, respectively, for which we use the short-hand notation of the last equality. The evaluation of $\hat{\chi}_{N}$ now becomes reduced to the evaluation of $D^{2} \Sigma$ on the right-hand side of (4.17).

As a first step, we complete the square in the exponent to obtain

$$
\Sigma(J, \bar{J})=e^{(J, C \bar{J})} \mathbb{E}_{C}\left(Z_{0}(\phi+C J, \bar{\phi}+C \bar{J}, \psi, \bar{\psi})\right) .
$$


In more detail, with $A=-\Delta+m^{2}=C^{-1}$,

$$
\begin{aligned}
\sum_{x \in \Lambda}\left(\tau_{\Delta, x}+m^{2} \tau_{x}\right)-(J, \bar{\phi})-(\phi, \bar{J}) & =(\phi, A \bar{\phi})+(\psi, A \bar{\psi})-(J, \bar{\phi})-(\phi, \bar{J}) \\
& =(\phi-C J, A(\bar{\phi}-C \bar{J}))+(\psi, A \bar{\psi})-(J, C \bar{J})
\end{aligned}
$$

and (4.18) follows with the translation $\phi \mapsto \phi+C J$ of the integration variable in the integral (3.18) defining the super-expectation $\mathbb{E}_{C}$. This translation of $\phi$ leaves $\psi=(2 \pi i)^{-1 / 2} d \phi$ unchanged. As we explain next, (4.18) can be expressed conveniently in terms of a notion of convolution.

By definition, any form $F$ in the algebra $\mathcal{N}=\mathcal{N}(\Lambda)$ of differential forms (see Section 3.1) is a linear combination of products of factors $\psi_{x_{i}}$ and $\bar{\psi}_{y_{i}}$, with $x_{i}, y_{i} \in \Lambda$ and with coefficients given by functions of $\phi$ and $\bar{\phi}$. We also define an algebra $\mathcal{N}^{\times}$with twice as many fields as $\mathcal{N}$, namely with boson fields $(\phi, \xi)$ and fermion fields $(\psi, \eta)$, where $\psi=(2 \pi i)^{-1 / 2} d \phi, \eta=(2 \pi i)^{-1 / 2} d \xi$. There are also the four corresponding conjugate fields. Given a form $F=f(\phi, \bar{\phi}) \psi^{x} \bar{\psi}^{y}$ (where $\psi^{x}$ denotes a product $\left.\psi_{x_{1}} \cdots \psi_{x_{j}}\right)$, we define

$$
\theta F=f(\phi+\xi, \bar{\phi}+\bar{\xi})(\psi+\eta)^{x}(\bar{\psi}+\bar{\eta})^{y},
$$

and extend this to a map $\theta: \mathcal{N} \rightarrow \mathcal{N}^{\times}$by linearity. Then we understand the map $\mathbb{E}_{C} \circ \theta: \mathcal{N} \rightarrow \mathcal{N}$ as the integration with respect to the fluctuation fields $\xi$ and $\eta$, with the fields $\phi$ and $\psi$ left fixed. This is like a conditional expectation. For example, if $F=f(\phi)$ is of degree- 0 , then

$$
\mathbb{E}_{C} \theta F=\mu_{C} * f=E_{C}(f(\phi+\xi) \mid \phi),
$$

where the right-hand side is the usual conditional expectation with respect to the Gaussian measure $d \mu_{C}$ defined in (3.19). However, in general, this is not usual probability theory, since $\mathbb{E}_{C} \circ \theta$ acts on the algebra of forms.

The expectation with the translated boson field $\phi$ in (4.18), with no corresponding translation of the fermion field $\psi$, can be expressed succinctly in terms of $\mathbb{E}_{C} \theta$ and projection onto the subspace of degree zero forms. To this end, let

$$
Z_{N}=\mathbb{E}_{C} \theta Z_{0}
$$

which is a differential form on $\mathbb{C}^{\Lambda}$. The subscript $N$ on $Z_{N}$ refers to the parameter defining the finite volume $\Lambda_{N}$. We denote the degree-0 part of $Z_{N}$ by $Z_{N}^{0}$, i.e., $Z_{N}^{0}$ is a function $Z_{N}^{0}: \mathbb{C}^{\Lambda} \rightarrow \mathbb{C}$. Then

$$
\Sigma(J, \bar{J})=e^{(J, C \bar{J})} Z_{N}^{0}(C J, C \bar{J}) .
$$

As mentioned already in (3.12), the partition function $\mathbb{E}_{C} Z_{0}$ is equal to 1 by supersymmetry. However it is much more challenging to understand the convolution $\mathbb{E}_{C} \theta Z_{0}$.

To express the susceptibility conveniently in terms of $Z_{N}^{0}$, observe that 1 is an eigenfunction of the covariance matrix $C$, namely

$$
C 1=\left(-\Delta+m^{2}\right)^{-1} 1=\frac{1}{m^{2}} 1 .
$$

With (4.23), this and $(1,1)=|\Lambda|$ imply that

$$
D^{2} \Sigma(0,0 ; 1,1)=(1, C 1)+D^{2} Z_{N}^{0}(0,0 ; C 1, C 1)=\frac{1}{m^{2}}|\Lambda|+\frac{1}{m^{4}} D^{2} Z_{N}^{0}(0,0 ; 1,1) .
$$


Thus, with (4.17), we obtain

$$
\hat{\chi}_{N}=\frac{1}{m^{2}}+\frac{1}{m^{4}} \frac{1}{|\Lambda|} D^{2} Z_{N}^{0}(0,0 ; 1,1)
$$

The representation (4.12) has the two free parameters $m^{2}$ and $z_{0}$, which define a division of the quadratic $\tau$ and $\tau_{\Delta}$ terms between the Gaussian expectation $\left(1+z_{0}\right) \mathbb{E}_{C}$ and the perturbation $V_{0}(\Lambda)$. The formula (4.26) holds for any choice of $m^{2}, z_{0}$ (and also any choice of $g_{0}, \nu_{0}$ ), but to study the behaviour near the critical point $\nu_{c}$, it is essential that this split be made exactly right. The correct critical choice is given in the following theorem, whose proof occupies the remainder of the paper. With this choice, we have $|\Lambda|^{-1} D^{2} Z_{N}^{0}(0,0 ; 1,1) \rightarrow 0$ as $N \rightarrow \infty$ in (4.26). In Section 4.3 below, we show that Theorem 1.1 is a consequence of Theorem 4.1 .

Theorem 4.1. Let $d=4$, and let $\delta>0$ be sufficiently small. There are continuous real-valued functions $\nu_{0}^{c}, z_{0}^{c}$, defined for $\left(m^{2}, g_{0}\right) \in[0, \delta)^{2}$ and continuously differentiable in $g_{0}$, and there is a continuous function $c\left(g_{0}\right)=1+O\left(g_{0}\right)$, such that for all $m^{2}, g_{0}, \hat{g}_{0} \in(0, \delta)$,

$$
\begin{aligned}
\hat{\chi}\left(m^{2}, g_{0}, \nu_{0}^{c}\left(m^{2}, g_{0}\right), z_{0}^{c}\left(m^{2}, g_{0}\right)\right) & =\frac{1}{m^{2}}, \\
\frac{\partial \hat{\chi}}{\partial \nu_{0}}\left(m^{2}, g_{0}, \nu_{0}^{c}\left(m^{2}, g_{0}\right), z_{0}^{c}\left(m^{2}, g_{0}\right)\right) & \sim-\frac{1}{m^{4}} \frac{c\left(\hat{g}_{0}\right)}{\left(\hat{g}_{0} \mathrm{~B}_{m^{2}}\right)^{\gamma}} \quad \text { as }\left(m^{2}, g_{0}\right) \rightarrow\left(0, \hat{g}_{0}\right) .
\end{aligned}
$$

The functions $\nu_{0}^{c}, z_{0}^{c}$ obey

$$
\nu_{0}^{c}\left(m^{2}, 0\right)=z_{0}^{c}\left(m^{2}, 0\right)=0, \quad \frac{\partial \nu_{0}^{c}}{\partial g_{0}}\left(m^{2}, g_{0}\right)=O(1), \quad \frac{\partial z_{0}^{c}}{\partial g_{0}}\left(m^{2}, g_{0}\right)=O(1),
$$

where $O(1)$ means that these derivatives are bounded on their whole domain by constants uniform in $\left(m^{2}, g_{0}\right)$.

\subsection{Change of parameters}

We have introduced six real variables $\left\{g, \nu, m^{2}, g_{0}, z_{0}, \nu_{0}\right\}$, and it is convenient in different contexts to switch perspective on which are dependent and which are independent variables. In particular, to deduce Theorem 1.1 from Theorem 4.1, we relate the parameters $\left(m^{2}, g_{0}, \nu_{0}^{c}, z_{0}^{c}\right)$ of $\hat{\chi}$ in (4.11) with the parameters $(g, \nu)$ of $\chi$. We summarise the different perspectives now. The six variables are constrained to satisfy the two equations in (4.3) as well as

$$
\nu_{0}=\nu_{0}^{c}\left(m^{2}, g_{0}\right), \quad z_{0}=z_{0}^{c}\left(m^{2}, g_{0}\right),
$$

with $\nu_{0}^{c}, z_{0}^{c}$ the functions of Theorem 4.1. In particular, (4.7) and (4.12) hold for such a choice.

Given $\left(m^{2}, g_{0}\right)$, (4.3) and (4.30) determine $g^{c}, \nu^{c}$ such that

$$
\left(g, \nu, \nu_{0}, z_{0}\right)=\left(g^{c}\left(m^{2}, g_{0}\right), \nu^{c}\left(m^{2}, g_{0}\right), \nu_{0}^{c}\left(m^{2}, g_{0}\right), z_{0}^{c}\left(m^{2}, g_{0}\right)\right),
$$

and (4.31) is continuous in $\left(m^{2}, g_{0}\right) \in[0, \delta)^{2}$ by Theorem 4.1. In Proposition 4.2(i) below, we show that, given $\left(m^{2}, g\right)$, we can determine

$$
\left(\nu, g_{0}, \nu_{0}, z_{0}\right)=\left(\nu^{*}\left(m^{2}, g\right), g_{0}^{*}\left(m^{2}, g\right), \nu_{0}^{*}\left(m^{2}, g\right), z_{0}^{*}\left(m^{2}, g\right)\right),
$$


continuously in $\left(m^{2}, g\right) \in\left[0, \delta_{1}\right)^{2}$, with (4.3) and (4.30) satisfied. In Proposition 4.2(ii) below, we show that given $\left(g, \nu_{c}+\varepsilon\right)$, we can determine

$$
\left(m^{2}, g_{0}, \nu_{0}, z_{0}\right)=\left(\tilde{m}^{2}(g, \varepsilon), \tilde{g}_{0}(g, \varepsilon), \tilde{\nu}_{0}(g, \varepsilon), \tilde{z}_{0}(g, \varepsilon)\right)
$$

so that (4.3) and (4.30) hold, with right-continuity as $\varepsilon \downarrow 0$. (We also expect continuity in $\varepsilon>0$, but have not proved it.)

Theorem 4.1 is stated in terms of parameters $\left(m^{2}, g_{0}\right)$ rather than the original parameters $(g, \nu)$ in (1.3). Let $\nu=\nu_{c}(g)+\varepsilon$, and let $\left(\tilde{m}^{2}, \tilde{g}_{0}, \tilde{\nu}_{0}, \tilde{z}_{0}\right)$ be given by (4.33). By (4.12) and Theorem 4.1, we have the equality

$$
\chi(g, \nu)=\left(1+\tilde{z}_{0}\right) \hat{\chi}\left(\tilde{m}^{2}, \tilde{g}_{0}, \tilde{\nu}_{0}, \tilde{z}_{0}\right)=\left(1+\tilde{z}_{0}\right) \frac{1}{\tilde{m}^{2}} .
$$

The right-hand side is $\left(1+\tilde{z}_{0}\right)$ times the susceptibility $\tilde{m}^{-2}$ of the non-interacting walk with killing rate $\tilde{m}^{2}$. Thus we have implemented the goal mentioned at the beginning of Section 4.1, i.e., to represent the susceptibility of the interacting model by that of a non-interacting model with renormalised parameters. Equation (4.34) is reminiscent of the renormalisation conditions imposed in the physics literature (e.g., [44, Chapter 5]). However, we stress that here (4.34) arises not by defining $\tilde{m}^{2}, \tilde{z}_{0}$ by the requirement that the equality holds, but rather that our method computes $\tilde{m}^{2}, \tilde{z}_{0}$ and we subsequently verify that the equality holds. The equality (4.34) is an identity, not merely an asymptotic formula, and thus it contains all information about the susceptibility, including not just the leading asymptotic behaviour but also all higher-order corrections.

Proposition 4.2. There exists $\delta_{1}>0$ such that the following hold.

(i) For $\left(m^{2}, g\right) \in\left[0, \delta_{1}\right)^{2}$, there exist

$$
\left(\nu, g_{0}, \nu_{0}, z_{0}\right)=\left(\nu^{*}\left(m^{2}, g\right), g_{0}^{*}\left(m^{2}, g\right), \nu_{0}^{*}\left(m^{2}, g\right), z_{0}^{*}\left(m^{2}, g\right)\right),
$$

continuous in $\left(m^{2}, g\right)$, such that (4.3) and (4.30) hold, and

$$
\begin{gathered}
\nu^{*}(0, g)=\nu_{c}(g), \quad \nu^{*}\left(m^{2}, g\right)>\nu_{c}(g) \quad\left(m^{2}>0\right), \\
g_{0}^{*}\left(m^{2}, g\right)=g+O\left(g^{2}\right), \quad \nu_{0}^{*}\left(m^{2}, g\right)=O(g), \quad z_{0}^{*}\left(m^{2}, g\right)=O(g) .
\end{gathered}
$$

(ii) For $(g, \varepsilon) \in\left[0, \delta_{1}\right)^{2}$, there exist

$$
\left(m^{2}, g_{0}, z_{0}, \nu_{0}\right)=\left(\tilde{m}^{2}(g, \varepsilon), \tilde{g}_{0}(g, \varepsilon), \tilde{z}_{0}(g, \varepsilon), \tilde{\nu}_{0}(g, \varepsilon)\right),
$$

right-continuous as $\varepsilon \downarrow 0$ (with g fixed), such that (4.3) and (4.30) hold, and

$$
\begin{gathered}
\tilde{m}^{2}(g, 0)=0, \quad \tilde{m}^{2}(g, \varepsilon)>0 \quad(\varepsilon>0), \\
\tilde{g}_{0}(g, \varepsilon)=g+O\left(g^{2}\right), \quad \tilde{\nu}_{0}(g, \varepsilon)=O(g), \quad \tilde{z}_{0}(g, \varepsilon)=O(g) .
\end{gathered}
$$

In the following proof, the construction of the maps (4.35) and (4.38) involves only elementary calculus. The proof does not use (4.28), but the identification of the critical point (which occurs in (4.36) and (4.39) $)$, as well as the proof of right-continuity of $\left(\tilde{m}^{2}, \tilde{g}_{0}, \tilde{\nu}_{0}, \tilde{z}_{0}\right)$ as $\varepsilon \downarrow 0$, uses (4.27). 
Proof. (i) For $\left(m^{2}, g_{0}\right) \in[0, \delta)^{2}$, set

$$
s\left(m^{2}, g_{0}\right)=\frac{g_{0}}{\left(1+z_{0}^{c}\left(m^{2}, g_{0}\right)\right)^{2}} .
$$

For fixed $m^{2}$, we show below that it is possible to construct an inverse to the map $g_{0} \mapsto s$, i.e., to show that there exists $\delta_{1}>0$ such that the equation $g=s\left(m^{2}, g_{0}\right)$ can be solved for $g_{0}=g_{0}^{*}\left(m^{2}, g\right)$ as a continuous function of $\left(m^{2}, g\right) \in\left[0, \delta_{1}\right)^{2}$. Given its existence, we set

$$
\nu_{0}^{*}=\nu_{0}^{*}\left(m^{2}, g\right)=\nu_{0}^{c}\left(m^{2}, g_{0}^{*}\left(m^{2}, g\right)\right), \quad z_{0}^{*}=z_{0}^{*}\left(m^{2}, g\right)=z_{0}^{c}\left(m^{2}, g_{0}^{*}\left(m^{2}, g\right)\right),
$$

and also define

$$
\nu^{*}=\nu^{*}\left(m^{2}, g\right)=\frac{\nu_{0}^{*}+m^{2}}{1+z_{0}^{*}} .
$$

Since $\nu_{0}^{c}$ and $z_{0}^{c}$ are continuous in $\left(m^{2}, g_{0}\right)$, and since $g_{0}^{*}$ is continuous in $\left(m^{2}, g\right)$, it is also the case that $z_{0}^{*}, \nu_{0}^{*}$, and $\nu^{*}$ are continuous in $\left(m^{2}, g\right)$, and it is immediate that (4.3) and (4.30) hold, and also that (4.37) holds. To show (4.36), it follows from (4.12) and (4.27) that

$$
\chi\left(g, \nu^{*}\left(m^{2}, g\right)\right)=\left(1+z_{0}^{*}\right) \hat{\chi}\left(m^{2}, g_{0}^{*}, \nu_{0}^{*}, z_{0}^{*}\right)=\left(1+z_{0}^{*}\right) \frac{1}{m^{2}} .
$$

In particular, $\chi\left(g, \nu^{*}\left(m^{2}, g\right)\right)<\infty$ if $m^{2}>0$ and therefore $\nu^{*}\left(m^{2}, g\right)>\nu_{c}(g)$ for $m^{2}>0$. Also, since $\chi\left(g, \nu^{*}\right) \uparrow \infty$ as $m^{2} \downarrow 0$, it follows from the continuity of $\nu^{*}$ that

$$
\nu_{c}(g)=\nu^{*}(0, g)=\inf \left\{\nu^{*}\left(m^{2}, g\right): m^{2} \in\left[0, \delta_{1}\right)\right\}
$$

To construct the inverse to $g_{0} \mapsto s$, we proceed as follows. By Theorem $4.1, \nu_{0}^{c}, z_{0}^{c}$ are continuous in $\left(m^{2}, g_{0}\right) \in[0, \delta)^{2}$, differentiable in $g_{0}$, and satisfy (4.29). Thus, with $z_{0}^{\prime}=\frac{\partial}{\partial g_{0}} z_{0}\left(m^{2}, g_{0}\right)$,

$$
\frac{\partial}{\partial g_{0}} s\left(m^{2}, g_{0}\right)=\frac{\left(1+z_{0}\right)^{2}-2 g_{0}\left(1+z_{0}\right) z_{0}^{\prime}}{\left(1+z_{0}\right)^{4}}=1+O\left(g_{0}\right)>0 \text {. }
$$

For sufficiently small $g_{*}>0, s$ is therefore a strictly increasing continuous function of $g_{0} \in\left[0, g_{*}\right)$ such that $\left|s\left(m^{2}, u\right)-s\left(m^{2}, v\right)\right| \geq\left(1-O\left(g_{*}\right)\right)|u-v|$ and hence, for $m^{2}$ fixed, $s\left(m^{2}, \cdot\right)$ is a continuously invertible map from $\left[0, g_{*}\right)$ onto the interval $\left[0, s\left(m^{2}, g_{*}\right)\right)$. Let $\left[0, \delta_{1}\right)$ be in the intersection over $m^{2}>0$ of the latter intervals. Since (4.46) holds uniformly in $m^{2}$, it follows that $\delta_{1}>0$. Therefore, $g=s\left(m^{2}, g_{0}\right)$ can be solved for $g_{0}$ as a function $g_{0}^{*}\left(m^{2}, g\right)$ for $g \in\left[0, \delta_{1}\right)$ and $g_{0}^{*}$ is continuous in $g$ for $m^{2}$ fixed. To prove that $g_{0}^{*}$ is jointly continuous in $\left(m^{2}, g\right)$ it suffices to show that when $\left(\hat{m}^{2}, \hat{g}\right) \rightarrow\left(m^{2}, g\right)$, then $\hat{g}_{0} \rightarrow g_{0}$, where $\hat{g}_{0}, g_{0}$ solve $s\left(\hat{m}^{2}, \hat{g}_{0}\right)-\hat{g}=0=s\left(m^{2}, g_{0}\right)-g$. This follows from $\left(1-O\left(g_{*}\right)\right)\left|\hat{g}_{0}-g_{0}\right| \leq\left|s\left(\hat{m}^{2}, \hat{g}_{0}\right)-s\left(\hat{m}^{2}, g_{0}\right)\right|=\left|\left(s\left(m^{2}, g_{0}\right)-s\left(\hat{m}^{2}, g_{0}\right)\right)+(\hat{g}-g)\right| \rightarrow 0$, since $s\left(\cdot, g_{0}\right)$ is continuous by (4.41) and the continuity of $z_{0}^{c}$.

(ii) We set

$$
\tilde{m}^{2}=\tilde{m}^{2}(g, \varepsilon)=\inf \left\{m^{2}>0: \nu^{*}\left(m^{2}, g\right)=\nu_{c}(g)+\varepsilon\right\} .
$$

By continuity of $\nu^{*}$, the infimum is attained and

$$
\nu_{c}(g)+\varepsilon=\nu^{*}\left(\tilde{m}^{2}(g, \varepsilon), g\right)
$$


The left-hand side of (4.48) converges to $\nu_{c}$ as $\varepsilon \downarrow 0$, and hence $\tilde{m}^{2}(g, \varepsilon) \downarrow 0$ as $\varepsilon \downarrow 0$ (if $\tilde{m}^{2}(g, \varepsilon$ ) had a nonzero accumulation point the right-hand side of (4.48) would not converge to $\nu_{c}$ ). We set

$$
\tilde{g}_{0}=\tilde{g}_{0}(g, \varepsilon)=g_{0}^{*}\left(\tilde{m}^{2}, g\right), \quad \tilde{\nu}_{0}=\tilde{\nu}_{0}(g, \varepsilon)=\nu_{0}^{*}\left(\tilde{m}^{2}, g\right), \quad \tilde{z}_{0}=\tilde{z}_{0}(g, \varepsilon)=z_{0}^{*}\left(\tilde{m}^{2}, g\right)
$$

and conclude that (4.3) and (4.30) hold. The desired properties of $\tilde{\nu}_{0}$ and $\tilde{z}_{0}$ follow from those of $\tilde{m}$ and $\tilde{g}_{0}$, and continuity of $\nu_{0}^{*}$ and $z_{0}^{*}$, and the proof is complete.

\subsection{Proof of Theorem 1.1 assuming Theorem 4.1}

The following elementary lemma is used in the proof of Theorem 1.1 .

Lemma 4.3. Let $\delta>0$. Suppose that $u:[0, \delta) \rightarrow[0, \infty)$ is continuous, differentiable on $(0, \delta)$, that $u(0)=0$ and $u(t)>0$ for $t>0$, and that

$$
u^{\prime}(t)=(-\log u(t))^{-\gamma}(1+o(1)) \quad(\text { as } t \downarrow 0) .
$$

Then

$$
u(t)=t(-\log t)^{-\gamma}(1+o(1)) \quad(\text { as } t \downarrow 0) .
$$

Proof. By hypothesis,

$$
\int_{0}^{t} u^{\prime}(t)(-\log u(t))^{\gamma} d t=\int_{0}^{t}(1+o(1)) d t=t(1+o(1)) .
$$

Note that $u(t)>0$ implies that $u^{\prime}(t)>0$ for small $t$, so $u$ is monotone. A change of variables, followed by integration by parts, gives

$$
\begin{aligned}
\int_{0}^{t} u^{\prime}(t)(-\log u(t))^{\gamma} d t & =\int_{0}^{u(t)}(-\log v)^{\gamma} d v \\
& =u(t)(-\log u(t))^{\gamma}\left(1+O\left((-\log u(t))^{-1}\right)\right) .
\end{aligned}
$$

The equality of the above two right-hand sides then gives

$$
u(t)(-\log u(t))^{\gamma}=t(1+o(1))
$$

Let $f(x)=x(-\log x)^{\gamma}$ and $g(y)=y(-\log y)^{-\gamma}$. Then $f$ and $g$ are approximate inverses in the sense that $f(g(y))=y(1+o(1))$. Thus $u(t)=t(-\log t)^{-\gamma}(1+o(1))$, and the proof is complete.

Proof of Theorem 1.1. Let $\nu=\nu_{c}(g)+\varepsilon$. We have observed already in (4.34) that

$$
\chi(g, \nu)=\left(1+\tilde{z}_{0}\right) \hat{\chi}\left(\tilde{m}^{2}, \tilde{g}_{0}, \tilde{\nu}_{0}, \tilde{z}_{0}\right)=\left(1+\tilde{z}_{0}\right) \frac{1}{\tilde{m}^{2}} .
$$

For the derivative, we apply (4.13), (4.28) and (4.55) to obtain, as $\nu \downarrow \nu_{c}$,

$$
\frac{\partial \chi}{\partial \nu}(g, \nu)=\left(1+\tilde{z}_{0}\right)^{2} \frac{\partial \hat{\chi}}{\partial \nu_{0}}\left(\tilde{m}^{2}, \tilde{g}_{0}, \tilde{\nu}_{0}, \tilde{z}_{0}\right) \sim-\chi^{2}(g, \nu) \frac{c_{0}(g)}{\left(\tilde{g}_{0} \mathrm{~B}_{\tilde{m}^{2}}\right)^{\gamma}} .
$$


The constant on the right-hand side is $c_{0}(g)=\lim _{\varepsilon \downarrow 0} c\left(\tilde{g}_{0}(g, \varepsilon)\right)$ which exists by right-continuity of $\tilde{g}_{0}$ at $\varepsilon=0$ for fixed $g$ and by continuity of $c$. Equation (4.13) relates a directional derivative of $\chi$ at the point $(g, \nu)$ to a directional derivative of $\hat{\chi}$ at any point $\left(m^{2}, g_{0}, \nu_{0}, z_{0}\right)$ related to $(g, \nu)$ by (4.3). Equation (4.56) is valid because it specialises (4.13) to $\left(\tilde{m}^{2}, \tilde{g}_{0}, \tilde{\nu}_{0}, \tilde{z}_{0}\right)$ which is related to $(g, \nu)$ by (4.3).

Now we drop $g$ from the notation. For $\nu>\nu_{c}$, let

$$
F(\nu)=\frac{1}{\chi(\nu)}
$$

By Proposition 4.2(ii), $\tilde{z}_{0}(\varepsilon)$ is continuous as $\varepsilon \downarrow 0$, and hence, by (4.55),

$$
F(\nu) \sim\left(1+\tilde{z}_{0}(0)\right)^{-1} \tilde{m}^{2} \quad\left(\text { as } \nu \downarrow \nu_{c}\right) .
$$

We set $F\left(\nu_{c}\right)=0$. By (4.56), together with (1.8), (4.58) and (4.40),

$$
\frac{\partial F}{\partial \nu}=-\frac{1}{\chi^{2}} \frac{\partial \chi}{\partial \nu} \sim \frac{c_{0}(g)}{\left(\tilde{g}_{0} \mathrm{~B}_{\tilde{m}^{2}}\right)^{\gamma}} \sim \frac{c_{0}(g)}{\left(\tilde{g}_{0}(0) \mathrm{b}\right)^{\gamma}}(-\log F(\nu))^{-\gamma} \quad\left(\text { as } \nu \downarrow \nu_{c}\right) .
$$

We apply Lemma 4.3 with $u(t)=\left(\tilde{g}_{0}(0) \mathrm{b}\right)^{\gamma} c_{0}(g)^{-1} F\left(\nu_{c}+t\right)$ to conclude from (4.59) that

$$
F\left(\nu_{c}+\varepsilon\right) \sim A_{g}^{-1} \varepsilon(-\log \varepsilon)^{-\gamma}
$$

with

$$
A_{g}=\frac{\left(\tilde{g}_{0}(0) \mathrm{b}\right)^{\gamma}}{c_{0}(g)}
$$

This is equivalent to (1.9). Since $c_{0}(g)=1+O\left(\tilde{g}_{0}(0)\right)=1+O(g)$ as $g \downarrow 0$ by (4.40), we obtain

$$
A_{g}=(g \mathbf{b})^{\gamma}(1+O(g))
$$

which proves (1.10) and completes the proof.

As a consequence of Theorems 1.1 and 4.1. we note in passing that the effective killing rate $m^{2}$ vanishes as the critical point is approached, i.e., as $\varepsilon=\nu-\nu_{c}(g) \downarrow 0$, according to the asymptotic formula

$$
m^{2} \sim \frac{1+z_{0}^{c}\left(0, g_{0}\right)}{A_{g}} \varepsilon\left(\log \varepsilon^{-1}\right)^{-\gamma} \quad \text { as } \varepsilon \downarrow 0 .
$$

\section{Renormalisation group}

In this section, we gather together some of the important ingredients of the renormalisation group analysis used in the proof of Theorem 4.1. 


\subsection{Progressive integration and covariance decomposition}

The proof of Theorem 4.1 is based on renormalisation group analysis of the integral on the righthand side of (4.9). We now explain a fundamental mechanism of our renormalisation group method: progressive Gaussian integration which enables a multi-scale analysis.

It is an elementary fact that if $X_{1} \sim N\left(0, \sigma_{1}^{2}\right)$ and $X_{2} \sim N\left(0, \sigma_{2}^{2}\right)$ are independent centred Gaussian random variables with respective variances $\sigma_{1}^{2}, \sigma_{2}^{2}$, then $X_{1}+X_{2} \sim N\left(0, \sigma_{1}^{2}+\sigma_{2}^{2}\right)$ (here $\sim$ denotes equality in distribution). In particular, if $X \sim N\left(0, \sigma_{1}^{2}+\sigma_{2}^{2}\right)$ then we can evaluate an expectation $E(f(X))$ by performing iterated Gaussian integrals, as

$$
E(f(X))=E\left(E\left(f\left(X_{1}+X_{2}\right) \mid X_{2}\right)\right) .
$$

The inner expectation on the right-hand side is conditional on $X_{2}$, and the outer expectation then averages over $X_{2}$. The elementary identity (5.1) extends to the super-expectation (3.18), as we describe next. Recall the map $\theta$ defined in (4.20).

Proposition 5.1. Let $F \in \mathcal{N}(\Lambda)$, and suppose that $C_{1}$ and $C^{\prime}$ are $\Lambda \times \Lambda$ matrices with positivedefinite Hermitian parts. Then

$$
\mathbb{E}_{C^{\prime}+C_{1}} \theta F=\left(\mathbb{E}_{C^{\prime}} \theta \circ \mathbb{E}_{C_{1}} \theta\right) F .
$$

For a proof, see [21, Proposition 2.6]. Equations (5.2) and (5.1) are closely related. To see this, consider the special case in which we set $\phi=\bar{\phi}=0$ and $\psi=\bar{\psi}=0$ in (5.2). In this case, (5.2) gives

$$
\mathbb{E}_{C^{\prime}+C_{1}} F=\mathbb{E}_{C^{\prime}}\left(\mathbb{E}_{C_{1}} \theta F\right),
$$

which is an abbreviation for the lengthier formula (in which we suppress the conjugate fields) expressed in the conventional notation for conditional expectations as

$$
\mathbb{E}_{C^{\prime}+C_{1}} F(\xi, \eta)=\mathbb{E}_{C^{\prime}}\left(\mathbb{E}_{C_{1}}\left(F\left(\xi^{\prime}+\xi_{1}, \eta^{\prime}+\eta_{1}\right) \mid \xi^{\prime}, \eta^{\prime}\right)\right) .
$$

As in Section 3.4, we set $C=\left(-\Delta+m^{2}\right)^{-1}$ where $\Delta$ is the Laplacian on the torus $\Lambda_{N}$ of period $L^{N}$. Our goal is to prove Theorem 4.1, which concerns the asymptotic behaviour of $\hat{\chi}_{N}$ of (4.9). In particular, we are interested in the limits $\Lambda_{N} \uparrow \mathbb{Z}^{d}$ and $m^{2} \downarrow 0$, so $C$ is an approximation to the operator $\left(-\Delta_{\mathbb{Z}^{d}}\right)^{-1}$. The kernel $\left[\left(-\Delta_{\mathbb{Z}^{d}}\right)^{-1}\right]_{x y}$ decays as $|x-y|^{-2}$ as $|x-y| \rightarrow \infty$ in dimension $d=4$, and such long-range correlations make the analysis difficult. The renormalisation group approach takes the long-range correlations into account progressively, by making a good decomposition of the covariance $C$ into a sum of terms with finite range, together with progressive integration. The covariance $\left(-\Delta_{\mathbb{Z}^{d}}+m^{2}\right)^{-1}$ with $m^{2}>0$ also arises in our analysis.

The progressive integration is based on finite-range decompositions of the covariances $C$ and $\left(-\Delta_{\mathbb{Z}^{d}}+m^{2}\right)^{-1}$. The decompositions we use are defined and discussed in detail in [6, Section 6.1], based on [3] (see also [15, 17]). In [3], it is shown that there are positive-definite covariances $\left(C_{j}\right)_{1 \leq j<\infty}$ on $\mathbb{Z}^{d}$ such that

$$
\left(-\Delta_{\mathbb{Z}^{d}}+m^{2}\right)^{-1}=\sum_{j=1}^{\infty} C_{j} .
$$

These covariances have the finite-range property that

$$
C_{j ; x, y}=0 \quad \text { if }|x-y| \geq \frac{1}{2} L^{j},
$$


and therefore $C_{j}$ can also be identified with a covariance on the torus of period $L^{N}$ when $N>j$. In addition, there is a positive-definite covariance $C_{N, N}$ on $\Lambda$ such that

$$
C=\left(-\Delta_{\Lambda}+m^{2}\right)^{-1}=\sum_{j=1}^{N-1} C_{j}+C_{N, N}
$$

The finite-range property gives rise to a factorisation property which is essential for the analysis of [24], upon which our results depend. To state the property, given $X \subset \Lambda$, recall that $\mathcal{N}(X)$ was defined in Section 3.1 as the subalgebra of $\mathcal{N}(\Lambda)$ consisting of forms that depend on the boson and fermion fields only at points in $X$. It follows from [21, Proposition 2.7] that for forms $F \in \mathcal{N}(X)$ and $G \in \mathcal{N}(Y)$ such that $\operatorname{dist}(X, Y)>\frac{1}{2} L^{j}$,

$$
\mathbb{E}_{C_{j}} \theta(F G)=\left(\mathbb{E}_{C_{j}} \theta F\right)\left(\mathbb{E}_{C_{j}} \theta G\right)
$$

so $F$ and $G$ are uncorrelated with respect to $\mathbb{E}_{C_{j}}$. This is an extension to the fermionic setting of the standard fact that uncorrelated Gaussian random variables are independent.

By (5.7) and Proposition 5.1.

$$
\mathbb{E}_{C} \theta F=\left(\mathbb{E}_{C_{N, N}} \theta \circ \mathbb{E}_{C_{N-1}} \theta \circ \cdots \circ \mathbb{E}_{C_{1}} \theta\right) F,
$$

and this expresses the expectation on the left-hand side as a progressive integration. The calculation of the expectation on the right-hand side of (4.26) provides the basis for our proof of Theorem 4.1. For simplicity, we sometimes write $C_{N}=C_{N, N}$. To compute the expectation (4.22), we use (5.9) to evaluate it progressively. Namely, if we define

$$
Z_{0}=e^{-V_{0}(\Lambda)}, \quad Z_{j+1}=\mathbb{E}_{C_{j+1}} \theta Z_{j} \quad(j<N)
$$

then, consistent with (4.22),

$$
Z_{N}=\mathbb{E}_{C} \theta Z_{0}
$$

Thus we are led to study the recursion $Z_{j} \mapsto Z_{j+1}$. To simplify the notation, we use the short-hand notation $\mathbb{E}_{j}=\mathbb{E}_{C_{j}}$, and leave implicit the dependence of the covariance $C_{j}$ on of the mass $m$.

In our analysis, the value $m^{2}=0$ corresponds to $\nu=\nu_{c}$. The fact that we work with covariances with $m^{2}>0$ is an important feature of our method because it permits the renormalisation group step to be carried out with good estimates indefinitely, not only for $\nu=\nu_{c}$ but also for $\nu>\nu_{c}$.

\subsection{Typical size of fields}

To control the progressive integration, we need good estimates on $C_{j}$. To state the estimates, we fix some $\delta>0$ and define mass intervals

$$
\mathbb{I}_{j}= \begin{cases}{[0, \delta)} & (j<N) \\ {\left[\delta L^{-2(N-1)}, \delta\right)} & (j=N) .\end{cases}
$$

It is shown in [6. Proposition 6.1] that for multi-indices $\alpha, \beta$ with $\ell^{1}$ norms $|\alpha|_{1},|\beta|_{1}$ at most some fixed value $p$, for $j \leq N$, for $m^{2} \in \mathbb{I}_{j}$, and for any $k \in \mathbb{N}$,

$$
\left|\nabla_{x}^{\alpha} \nabla_{y}^{\beta} C_{j ; x, y}\right| \leq c\left(1+m^{2} L^{2(j-1)}\right)^{-k} L^{-(j-1)\left(2[\phi]+\left(|\alpha|_{1}+|\beta|_{1}\right)\right)},
$$




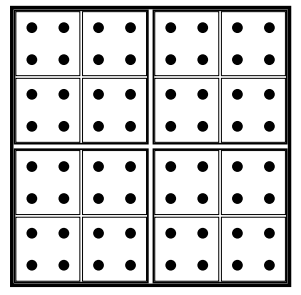

Figure 1: Illustration of $\mathcal{B}_{j}(\Lambda)$ for $j=0,1,2,3$ when $d=2, N=3, L=2$.

where $c=c(k)$ depends on $k$ and $\delta$ but is independent of $j$ and $m^{2}$, and the estimate is for $C_{N, N}$ when $j=N$. Here $\nabla_{x}^{\alpha}=\nabla_{x_{1}}^{\alpha_{1}} \ldots \nabla_{x_{d}}^{\alpha_{d}}$ for a multi-index $\alpha=\left(\alpha_{1}, \ldots, \alpha_{d}\right)$, where $\nabla_{x_{k}}$ denotes the finite-difference operator $\nabla_{x_{k}} f(x, y)=f\left(x+e_{k}, y\right)-f(x, y)$. The number $[\phi]=\frac{1}{2}(d-2)$ is referred to as the scaling dimension or engineering dimension of the field, or, more briefly, simply as the field's dimension. Throughout this paper, the parameter $\delta>0$ in (5.12) is fixed and all constants are allowed to depend on it. For other reasons, we sometimes assume that $\delta>0$ is small. We take limits in the order $N \rightarrow \infty$, and then $m^{2} \downarrow 0$, so that the condition $m^{2} \geq \delta L^{-2(N-1)}$ required for the validity of (5.13) is satisfied. Moreover, throughout the remainder of this paper, we tacitly assume that $L$ is sufficiently large. This is needed, in particular, for the results of [23, 24], on which our results rely.

In the expectation $Z_{j+1}=\mathbb{E}_{j+1} \theta Z_{j}$, on the right-hand side we may write $\phi_{j}=\phi_{j+1}+\xi_{j+1}$, as in (4.20), and similarly for $\bar{\phi}_{j}, d \phi_{j}, d \bar{\phi}_{j}$. The expectation $\mathbb{E}_{j+1} \theta$ integrates out $\xi_{j+1}, \bar{\xi}_{j+1}, d \xi_{j+1}, d \bar{\xi}_{j+1}$ leaving dependence of $Z_{j+1}$ on $\phi_{j+1}, \bar{\phi}_{j+1}, d \phi_{j+1}, d \bar{\phi}_{j+1}$. This process is repeated. The $\xi_{j}$ fields that are integrated out are called fluctuation fields. The field $\phi_{j}$ is thus the variable in a Gaussian super-expectation with the remaining covariance $C_{\geq j+1}=\sum_{k=j+1}^{N} C_{k}$. By (3.19) $), \mathbb{E}_{\geq j+1}\left|\phi_{j, x}\right|^{2}=$ $C_{\geq j+1 ; x, x}$. The bounds of (5.13) suggest that $C_{\geq j+1 ; x, x} \approx C_{j+1 ; x, x}$, so that the typical size of the field $\phi_{j ; x}$ is of order $L^{-j[\phi]}$. Moreover, (5.13) also indicates that the derivative $\nabla_{x_{k}} \phi_{j ; x}$ is typically smaller than the field itself by a factor $L^{-j}$, so that $\phi_{j}$ remains approximately constant over a distance $L^{j}$. It is less familiar to think of the size of a differential form, but motivated by

$$
\mathbb{E}_{j+1}\left(\bar{\psi}_{x} \psi_{y}\right)=\mathbb{E}_{j+1}\left(\bar{\phi}_{x} \phi_{y}\right)=C_{j+1 ; x y}
$$

we can apply the same heuristics as for $\phi$ to the fermion field $\psi$.

\subsection{Polymers and relevant directions}

To make a systematic analysis of the fluctuation fields with covariance satisfying (5.6) and (5.13), we introduce nested pavings of the torus $\Lambda=\Lambda_{N}=\mathbb{Z}^{d} / L^{N} \mathbb{Z}^{d}$ by sets of blocks $\mathcal{B}_{j}$ on scales $j=0, \ldots, N$. See Figure 1, The blocks in $\mathcal{B}_{0}$ are simply the points in $\Lambda$. The blocks in $\mathcal{B}_{1}$ form a disjoint paving of $\Lambda$ by boxes of side $L$. More generally, each block in $\mathcal{B}_{j}$ has side length $L^{j}$ and consists of $L^{d}$ disjoint blocks in $\mathcal{B}_{j-1}$. The following definition makes this more formal, and also introduces the concept of a polymer, which has a long history in statistical mechanics going back to the important paper [37] (these polymers have nothing to do with long chain molecules or random walks). 
Definition 5.2. For each $j=0,1, \ldots, N$, the torus $\Lambda$ is paved in a natural way by $L^{N-j}$ disjoint $d$-dimensional cubes of side $L^{j}$. The cube that contains the origin at the corner has the form

$$
\left\{x \in \Lambda: x_{i}=0,1, \ldots, L^{j}-1 \forall i=1, \ldots, d\right\}
$$

and all the other cubes are translates of this one by vectors in $L^{j} \mathbb{Z}^{d}$. We call these cubes $j$-blocks, or blocks for short, and denote the set of $j$-blocks by $\mathcal{B}_{j}=\mathcal{B}_{j}(\Lambda)$. A union of $j$-blocks is called a polymer or $j$-polymer, and the set of $j$-polymers is denoted $\mathcal{P}_{j}=\mathcal{P}_{j}(\Lambda)$.

For a block $B \in \mathcal{B}_{j}$, the considerations in Section 5.2 concerning the typical size of $\phi_{j}$ suggest that, at each of the $L^{d j}$ points $x \in B, \phi_{j, x}$ has typical size of order $L^{-j[\phi]}$, and hence

$$
\sum_{x \in B} \phi_{j, x}^{p} \approx L^{d j} L^{-p j[\phi]}=L^{(d-p[\phi]) j}
$$

The above right-hand side is relevant (growing exponentially in $j$ ) for $p[\phi]<d$, irrelevant (decaying exponentially in $j$ ) for $p[\phi]>d$, and marginal (neither growing or decaying) for $p[\phi]=d$. Since $\tau_{x}=\phi_{x} \bar{\phi}_{x}+\psi_{x} \bar{\psi}_{x}$ is quadratic in the fields, it corresponds to $p=2$, so $p[\phi]=2[\phi]=d-2<d$ and $\tau_{x}$ is relevant in all dimensions. Similarly, $\tau_{x}^{2}$ corresponds to $p=4$ with $p[\phi]=4[\phi]=2 d-4$, so $\tau_{x}^{2}$ is irrelevant for $d>4$, marginal for $d=4$, and relevant for $d<4$. Also, $\tau_{\Delta, x}$ is marginal in all dimensions. In fact, by [6, Lemma 5.3], any sum of local field monomials in $\phi$ and $\psi$ that are relevant or marginal, Euclidean invariant, and that obey the additional symmetry between boson and fermions called supersymmetry (see [6, Section 5.2] for details) is of the same form as $V_{0}$ of (4.5), plus an additional term $\tau_{\nabla \nabla}$ (the use of summation by parts to avoid inclusion of the term $\tau_{\nabla \nabla}$ is discussed in [6, Section 4.2]).

Essentially based on this observation, the general approach of Wilson [62] suggests that, for $d=4$ the map $Z_{j} \mapsto Z_{j+1}$ is qualitatively well approximated by a 3 -dimensional map $\left(g_{j}, z_{j}, \mu_{j}\right) \mapsto$ $\left(g_{j+1}, z_{j+1}, \mu_{j+1}\right)$ such that

$$
Z_{j} \approx e^{-V_{j}(\Lambda)}
$$

where $V_{j}$ has the same form as $V_{0}$ with renormalised coupling constants $g_{j}, z_{j}, \mu_{j}$. Furthermore, Wilson's general approach suggests that, given $g_{0}>0$, one can find $z_{0}, \mu_{0}$ such that $\left(g_{j}, z_{j}, \mu_{j}\right) \rightarrow 0$, i.e., $Z_{j} \approx 1$ as $j \rightarrow \infty$. This is the famous observation of infrared asymptotic freedom. In the context of Section 4.1, it corresponds to the approximation of the interacting model by the noninteracting model. Our method gives a rigorous meaning to the approximation, by introducing a codimension-3 error coordinate $K_{j}$ which rigorously keeps track of all errors in the approximation. The introduction of $K_{j}$ trades the linear but nonlocal evolution of $Z_{j}$ given by (5.10) for a nonlinear but local evolution of $\left(V_{j}, K_{j}\right)$. The evolution of $\left(V_{j}, K_{j}\right)$ is addressed in Sections 6 [7 below.

Our construction of the evolution map in [24] makes important use of the finite-range property of the covariance decomposition. This can be contrasted with the block spin method [33, 34], in which the fluctuation covariances $C_{j}$ are chosen such the fields $\phi_{j}$ are constant over the blocks in $\mathcal{B}_{j}(\Lambda)$. Block spin covariances decay exponentially, but do not have the finite-range property (5.6). In our setup, fields are only approximately constant over blocks by (5.13), but this is compensated by the independence property (5.8) which allows for an effective construction of a renormalisation group map, by using independence rather than cluster expansion. 


\subsection{Interaction functional}

Motivated by the discussion in Section 5.3, we define $\mathcal{V}=\mathbb{R}^{3}$ and identify $\mathcal{V}$ with the vector space of local field polynomials

$$
V=g \tau^{2}+\nu \tau+z \tau_{\Delta}
$$

where $(g, \nu, z) \in \mathbb{R}^{3}$. Rather than the coupling constant $\nu=\nu_{j}$ we often use its rescaled version

$$
\mu_{j}=L^{2 j} \nu_{j}
$$

The reason for this rescaling is that, according to the heuristics discussed around (5.16), the sizes of $\tau^{2}, \tau_{\Delta}$ are comparable with that of $L^{-2 j} \tau$ rather than with that of $\tau$. There is no distinction between $\mu_{0}$ and $\nu_{0}$, so the two are interchangeable. The parameters $(g, z, \nu)$ in $V$ are designed to track the relevant and marginal directions in the dynamical system determined by the renormalisation group flow. The norm we use on $\mathcal{V}$ is the scale-dependent maximum norm on $\mathbb{R}^{3}$ with respect to the rescaled coordinate $\mu$, i.e.,

$$
\|V\|_{\mathcal{V}}=\max \{|g|,|z|,|\mu|\}=\max \left\{|g|,|z|, L^{2 j}|\nu|\right\} .
$$

Given a polymer $X \in \mathcal{P}_{j}(\Lambda)$ as in Definition [5.2, we write $V(X)$ for the form

$$
V(X)=\sum_{x \in X}\left(g \tau_{x}^{2}+\nu \tau_{x}+z \tau_{\Delta, x}\right)
$$

In [6, Section 3.5], for $X \in \mathcal{P}_{j}(\Lambda)$ we define a field functional $W_{j}(V, X) \in \mathcal{N}$ as an explicit quadratic function of $V \in \mathcal{V}$. Given $V \in \mathcal{V}, B \in \mathcal{B}_{j}(\Lambda)$, and $X \in \mathcal{P}_{j}(\Lambda)$, we define the interaction functional $I_{j}: \mathcal{P}_{j}(\Lambda) \rightarrow \mathcal{N}(\Lambda)$ by

$$
I_{j}(V, B)=e^{-V(B)}\left(1+W_{j}(V, B)\right), \quad I_{j}(V, X)=\prod_{B \in \mathcal{B}_{j}(X)} I_{j}(V, B) .
$$

For simplicity, we often write $I_{j}(X)=I_{j}(V, X)$, with the coordinate $V$ left implicit. The precise definitions of $W_{j}(V, X)$ and $I_{j}(V, B)$ do not play a major role in this paper, but they do play a role in results from [6, 23, 24] upon which our analysis depends. The interaction functional $I_{j}$ has the following important properties. In their statement, the enlargement $B^{+}$of a block $B$ is the union of $B$ and its neighbouring blocks.

- Field locality: $I_{j}(B) \in \mathcal{N}\left(B^{+}\right)$for each block $B \in \mathcal{B}_{j}\left(\Lambda_{N}\right)$;

- Symmetry: $I_{j}$ is supersymmetric and Euclidean invariant;

- Block factorisation: $I_{j}(X)=\prod_{B \in \mathcal{B}_{j}(X)} I_{j}(B)$.

By definition, $W_{0}=0$, so $I_{0}(V, X)=e^{-V(X)}$ for all $X \subset \Lambda$. With $V_{0}$ defined by (4.5), it follows from (5.10) that

$$
Z_{0}=I_{0}(\Lambda)=I_{0}\left(V_{0}, \Lambda\right)
$$


The definition (5.22) is motivated in [6, Section 2]. In short, given $V$ as in (5.18), it is shown in [6. Section 2] that there is an explicit choice $V_{\mathrm{pt}}$ of the same form as $V$ but with renormalised coupling constants, such that as a formal power series in $V$,

$$
\mathbb{E}_{j+1} \theta I_{j}(V, \Lambda)=I_{j+1}\left(V_{\mathrm{pt}}, \Lambda\right)+O\left(V^{3}\right) .
$$

This shows that the form of $I$ is stable in the sense that integrating out a fluctuation field is approximately the same as replacing $V$ by $V_{\mathrm{pt}}$. However, the error in (5.24) fails to be uniform in the size of $\Lambda$. To obtain an approximation that is uniform in $\Lambda$, we use the localised form (5.22).

The factorised version (5.22), together with our choice of the side length of $B$ to be larger than the range of the covariance, allows us to take advantage of independence of fields on polymers that do not touch. An expression of this is (5.8), which implies that whenever $X_{1}, X_{2} \in \mathcal{P}_{j}(\Lambda)$ do not touch, and $F\left(X_{i}\right) \in \mathcal{N}\left(X_{i}\right)$ for $i=1,2$, then

$$
\mathbb{E}_{j} \theta\left(F\left(X_{1}\right) F\left(X_{2}\right)\right)=\left(\mathbb{E}_{j} \theta F\left(X_{1}\right)\right)\left(\mathbb{E}_{j} \theta F\left(X_{2}\right)\right)
$$

This factorisation property can be combined with a perturbative calculation on individual polymers, together with a careful control of errors in the perturbative calculation. Large parts of [6, 23, 24] are concerned with such matters.

\subsection{Circle product and error coordinate}

Given $F_{1}, F_{2}: \mathcal{P}_{j} \rightarrow \mathcal{N}$, we define their circle product $F_{1} \circ F_{2}: \mathcal{P}_{j} \rightarrow \mathcal{N}$ by

$$
\left(F_{1} \circ F_{2}\right)(Y)=\sum_{X \in \mathcal{P}_{j}: X \subset Y} F_{1}(X) F_{2}(Y \backslash X), \quad \text { for } Y \in \mathcal{P}_{j}
$$

The terms corresponding to $X=\varnothing$ and $X=Y$ are included in the summation on the right-hand side, and we assume that $F(\varnothing)=1$ for all $F: \mathcal{P}_{j} \rightarrow \mathcal{N}$. The circle product depends on the scale $0 \leq j \leq N$, but we leave this dependence implicit. It is an associative and commutative product, since $\mathcal{N}$ has these properties. The identity element for the circle product is $\mathbb{1}_{\varnothing}(X)=\mathbb{1}_{X=\varnothing}$, i.e., $\left(F \circ \mathbb{1}_{\varnothing}\right)(Y)=F(Y)$ for all $F$ and $Y$. by

Let $K_{0}: \mathcal{P}_{0} \rightarrow \mathcal{N}$ be the identity element $K_{0}=\mathbb{1}_{\varnothing}$. Then $Z_{0}=I_{0}\left(V_{0}, \Lambda\right)$ of (5.10) is also given

$$
Z_{0}=I_{0}(\Lambda)=\left(I_{0} \circ K_{0}\right)(\Lambda) .
$$

Our procedure is to maintain this form,

$$
Z_{j}=\left(I_{j} \circ K_{j}\right)(\Lambda) \quad \text { with } \quad K_{j}: \mathcal{P}_{j}(\Lambda) \rightarrow \mathcal{N}(\Lambda),
$$

in the recursion $Z_{j} \mapsto Z_{j+1}=\mathbb{E}_{j+1} \theta Z_{j}$ of (5.10), with the initial condition given by (5.27). With $I_{j}=I_{j}\left(V_{j}\right)$, the action of $\mathbb{E}_{j+1} \theta$ on $Z_{j}$ is then expressed as the renormalisation group map

$$
\left(V_{j}, K_{j}\right) \mapsto\left(V_{j+1}, K_{j+1}\right) .
$$

The map (5.29) depends on the scale $j$ and the volume parameter $N$. To achieve this, given $V_{j} \in \mathcal{V}$ and $K_{j}: \mathcal{P}_{j}(\Lambda) \rightarrow \mathcal{N}(\Lambda)$, we seek to define $V_{j+1} \in \mathcal{V}$ and $K_{j+1}: \mathcal{P}_{j+1}(\Lambda) \rightarrow \mathcal{N}(\Lambda)$ in such a way that

$$
Z_{j+1}=\mathbb{E}_{j+1} \theta\left(I_{j} \circ K_{j}\right)(\Lambda)=\left(I_{j+1} \circ K_{j+1}\right)(\Lambda)
$$


Then $Z_{j}=\left(I_{j} \circ K_{j}\right)(\Lambda)$ retains its form under progressive integration. The representation (5.28) is by no means unique: given $Z_{j}$ there are many choices of $I_{j}, K_{j}$ such that (5.30) holds. For example, a trivial choice is given by $K_{j}(X)=\mathbb{1}_{X=\Lambda} Z_{j}(X)$ along with any $I_{j}$ for which $I_{j}(\varnothing)=1$.

It is crucial to make a careful choice of the representation in order to obtain useful estimates valid at all scales. A choice of the map $\left(V_{j}, K_{j}\right) \mapsto\left(V_{j+1}, K_{j+1}\right)$ that leads to good estimates is explicitly constructed in [24]. This choice is such that $K_{j}=O\left(\left\|V_{j}\right\|^{3}\right)$ holds in a certain precise sense, so that $K_{j}$ can be regarded as a third-order error term. We discuss this in more detail in the next section, deferring substantial details to [24].

Substantial details underlying our analysis are developed in the series of papers [6, 7, 21, 24, One important aspect, which is worthy of mention despite not playing a visible role in the present paper, is the operator Loc defined and analysed in [22]. The operator Loc extracts from an arbitrary element of $\mathcal{N}$ its relevant and marginal parts, in the form of a local polynomial in the fields. Although Loc does not make a direct appearance in the present paper, it plays a crucial role in results from [6, 23, 24 upon which we rely here. The local polynomial $V_{j+1}$ is created from $\left(V_{j}, K_{j}\right)$ using Loc, which is used to incorporate the relevant and marginal parts of $W_{j}$ and $K_{j}$ into $V_{j+1}$.

\section{Renormalisation group map}

In this section, we discuss the definition and properties of the renormalisation group map $\left(V_{j}, K_{j}\right) \mapsto$ $\left(V_{j+1}, K_{j+1}\right)$ in more detail. Our discussion summarises results of [6, 23, 24], where further details can be found. The observable fields $\sigma, \bar{\sigma}$ that appear in those references play no role in this paper, and can be set equal to zero for our present needs. The observable fields are needed for the analysis of critical correlation functions in [5, 60].

Both local and global aspects are important for the repeated application of the renormalisation group map. In this section, we focus on the local aspect, which concerns a single application under appropriate assumptions on $\left(V_{j}, K_{j}\right)$. We give precise meaning to the notion that $V_{j}$ is a secondorder approximation of $Z_{j}$ and that $K_{j}$ is a third-order remainder. The global aspect is discussed in Section 7, this is the requirement that the assumptions on $\left(V_{j}, K_{j}\right)$ continue to remain valid as the two scales $j$ and $N$ increase indefinitely. It is the global aspect that requires the careful choice of the initial parameters $z_{0}$ and $\nu_{0}$ in Theorem 4.1.

\subsection{Perturbative quadratic flow}

In [6. (4.11)], an explicit quadratic map $\varphi_{\mathrm{pt}, j}^{(0)}: \mathbb{R}^{3} \rightarrow \mathbb{R}^{3}$ is defined and discussed. This map defines

$V_{\mathrm{pt}, j+1}^{(0)}=\varphi_{\mathrm{pt}, j}^{(0)}\left(V_{j}\right)$ in such a way that the formal power series identity (5.24) is satisfied. The map $\varphi_{\mathrm{pt}, j}^{(0)}$ is a second-order approximation to the $V$-component of the full renormalisation group map, $\left(V_{j}, K_{j}\right) \mapsto\left(V_{j+1}, K_{j+1}\right)$. It gives the dominant contribution to the renormalisation group map, and it must be understood in detail.

It is useful to re-express the $V$-component of the renormalisation group map in transformed coordinates. In [6, Proposition 4.3], we define quadratic polynomials $T_{j}: \mathbb{R}^{3} \rightarrow \mathbb{R}^{3}$ and consider the change of variables $V_{j} \mapsto \check{V}_{j}=T_{j}\left(V_{j}\right)$. The transformation $T_{j}$ satisfies

$$
T_{0}(V)=V, \quad T_{j}(V)=V+O\left(\|V\|^{2}\right),
$$


with error estimate uniform in $j$. Since the $T_{j}$ are polynomials, this implies that they are invertible in a neighbourhood of 0 that is independent of $j$. The composite maps $T_{j+1} \circ \varphi_{\mathrm{pt}, j}^{(0)} \circ T_{j}^{-1}$ are equal, up to an error $O\left(\|V\|^{3}\right)$, to $\bar{\varphi}_{j}: \mathbb{R}^{3} \rightarrow \mathbb{R}^{3}$, given explicitly by $\left(\bar{g}_{j+1}, \bar{z}_{j+1}, \bar{\mu}_{j+1}\right)=\bar{\varphi}_{j}\left(\bar{g}_{j}, \bar{z}_{j}, \bar{\mu}_{j}\right)$ with

$$
\begin{aligned}
\bar{g}_{j+1} & =\bar{g}_{j}-\beta_{j} \bar{g}_{j}^{2}, \\
\bar{z}_{j+1} & =\bar{z}_{j}-\theta_{j} \bar{g}_{j}^{2}, \\
\bar{\mu}_{j+1} & =L^{2} \bar{\mu}_{j}\left(1-\gamma \beta_{j} \bar{g}_{j}\right)+\eta_{j} \bar{g}_{j}-\xi_{j} \bar{g}_{j}^{2}-\pi_{j} \bar{g}_{j} \bar{z}_{j},
\end{aligned}
$$

where $\beta_{j}, \theta_{j}, \eta_{j}, \xi_{j}, \pi_{j}$ are real coefficients defined precisely in [․, (3.24), (3.27) $\left.-(3.28)\right]$. These coefficients, and also those of the transformations $T_{j}$, depend continuously on the mass $m^{2}$ appearing in the covariance decomposition of Section 5.1, but are independent of the size $N$ of the torus.

The effect of the transformation $T_{j}$ is to triangularise the evolution equation to second order: the $\bar{g}$-equation does not depend on $\bar{z}$ or $\bar{\mu}$, the $\bar{z}$-equation depends only on $\bar{g}$, and the $\bar{\mu}$-equation depends both on $\bar{g}$ and $\bar{z}$. This second-order triangularisation is the natural coordinate system to study the evolution of $V_{j}$. For example, we emphasise the explicit occurrence of the parameter $\gamma=\frac{1}{4}$ in (6.4) which, in Section 8, gives rise to the power of the logarithm in Theorem 1.1. This parameter is not apparent in the original equations before transformation by $T_{j}$ (cf. [6, (3.31)] where an additional term proportional to $g \mu$ appears).

The sequence $\left(\beta_{j}\right)_{0 \leq j<\infty}$ plays a key role in the analysis. It is defined in [6, (3.27)] by

$$
\beta_{j}=8 \sum_{x \in \mathbb{Z}^{d}}\left(w_{j+1, x}^{2}-w_{j, x}^{2}\right), \quad \text { with } w_{j, x}=\sum_{i=1}^{j} C_{i ; 0, x} .
$$

The $m^{2}$-dependence of $\beta_{j}$ is suppressed in the notation. Since each $C_{i}$ is positive-definite, the above definition implies that $\beta_{j}>0$ for all $j$. Moreover, it is shown in [6, Lemma 6.3] that $\lim _{j \rightarrow \infty} \beta_{j}=\log L / \pi^{2}$ for $m^{2}=0$, so $\beta_{j}$ is bounded away from 0 for sufficiently large $j$. On the other hand, for $m^{2}>0$, by (5.13), $\beta_{j}$ decays extremely rapidly to 0 for $j \geq j_{m}$ where $j_{m}$ is the mass scale, i.e., the smallest $j$ such that $L^{2 j} m^{2} \geq 1$. The mass scale is natural for our specific sequence (6.5), but we apply below a general dynamical system result from [7], in which there is no explicit mass parameter with which to define a mass scale.

To prepare for the application of the main result of [7], given $\Omega>1$ we define the $\Omega$-scale $j_{\Omega} \in \mathbb{N} \cup\{\infty\}$, by

$$
j_{\Omega}=j_{\Omega}\left(m^{2}\right)=\inf \left\{k \geq 0:\left|\beta_{j}\right| \leq \Omega^{-(j-k)}\|\beta\|_{\infty} \text { for all } j\right\} .
$$

For the remainder of the paper, we fix $\Omega>1$ arbitrarily, e.g., $\Omega=2$. According to [6, Proposition 4.4,$j_{m}$ and $j_{\Omega}$ are equivalent for the sequence (6.5), in the sense that $\left|j_{m}-j_{\Omega}\right|$ is bounded uniformly as $m^{2} \downarrow 0$. Thus we may regard the mass scale and $\Omega$-scale as essentially equivalent. We define

$$
\chi_{j}=\chi_{j}\left(m^{2}\right)=\Omega^{-\left(j-j_{\Omega}\right)_{+}} .
$$

In [6, Proposition 4.4], it is verified that the coefficients $\theta_{j}, \eta_{j}, \xi_{j}, \pi_{j}$ are bounded by $O\left(\chi_{j}\right)$ and that they depend continuously on $m^{2}$. In particular, it is shown that the following two assumptions are satisfied.

Assumption (A1). The sequence $\beta$ : The sequence $\left(\beta_{j}\right)$ is bounded, namely $\beta_{\max }=\sup _{j \in \mathbb{N}_{0}}\left|\beta_{j}\right|<$ $\infty$. There exists $c>0$ such that $\beta_{j} \geq c$ for all but $c^{-1}$ values of $j \leq j_{\Omega}$. 
Assumption (A2). The other parameters of $\bar{\varphi}$ : Each of $\theta_{j}, \eta_{j}, \xi_{j}$, and $\pi_{j}$ is bounded in absolute value by $O\left(\chi_{j}\right)$, with a constant that is independent of both $j$ and $j_{\Omega}$.

The above Assumption (A1) is the same as [7, Assumption (A1)], and the above Assumption (A2) is a specialised form of [7. Assumption (A2)] (where in the notation of [7, $\zeta_{j}=0$, $v_{j}^{g g}=\xi_{j}, v_{j}^{g z}=\pi_{j}, v_{j}^{g \mu}=L^{2} \gamma \beta_{j}$, and $\left.v_{j}^{z z}=v_{j}^{z \mu}=0\right)$. We apply Assumptions (A1-A2) in the proof of Proposition 7.1 below, where we apply the main result of [7].

We interpret the scale parameter $j$ as time, and thus view $\bar{\varphi}=\left(\bar{\varphi}_{j}\right)_{j \in \mathbb{N}_{0}}$ as a time-dependent dynamical system on $\mathbb{R}^{3}$ which we call the quadratic flow. The exponential decay of the coefficients beyond $j_{\Omega}$ has the interpretation that the evolution essentially stops at $j=j_{\Omega}$. The dynamical system $\bar{\varphi}_{j}$ has the fixed point $(0,0,0)$. This fixed point is not hyperbolic: there are two unit eigenvalues of $D \bar{\varphi}_{j}(0)$ corresponding to the $g$-and $z$-equations. In [7], such non-hyperbolic fixed points are studied in a general infinite-dimensional setting. The flow of $\bar{\varphi}$ near the fixed point 0 is elementary, and can be understood via explicit analysis using the triangular structure of the equations (6.2)-(6.4). In particular, the following proposition is a special case of [7, Proposition 1.2]. In its statement, $z_{\infty}$ denotes the limit $z_{\infty}=\lim _{j \rightarrow \infty} z_{j}$, and similarly for $\mu_{\infty}$.

Proposition 6.1. If $\bar{g}_{0}>0$ is sufficiently small, then there exists a unique global flow $\bar{V}=$ $(\bar{V})_{j \in \mathbb{N}_{0}}=\left(\bar{g}_{j}, \bar{z}_{j}, \bar{\mu}_{j}\right)_{j \in \mathbb{N}_{0}}$ of $\bar{\varphi}$, i.e., a solution to $\bar{V}_{j+1}=\bar{\varphi}_{j}\left(V_{j}\right)$ defined for all $j \in \mathbb{N}_{0}$, with initial condition $\bar{g}_{0}$ and final condition $\left(\bar{z}_{\infty}, \bar{\mu}_{\infty}\right)=(0,0)$. This flow satisfies, for any real $p \in[1, \infty)$,

$$
\chi_{j} \bar{g}_{j}^{p}=O\left(\frac{\bar{g}_{0}}{1+\bar{g}_{0} j}\right)^{p}, \quad \bar{z}_{j}=O\left(\chi_{j} \bar{g}_{j}\right), \quad \bar{\mu}_{j}=O\left(\chi_{j} \bar{g}_{j}\right),
$$

with constants independent of $j_{\Omega}$ and $\bar{g}_{0}$, and dependent on $p$ in the first bound. Furthermore, $\bar{V}_{j}$ is continuously differentiable in the initial condition $\bar{g}_{0}$ and continuous in the mass parameter $m^{2}$, for every $j \in \mathbb{N}_{0}$.

The main difficulty in the analysis of the renormalisation group map (5.29) lies in the control of the non-perturbative coordinate $K_{j}: \mathcal{P}_{j}(\Lambda) \rightarrow \mathcal{N}(\Lambda)$, and we address this next.

\subsection{Non-perturbative coordinate}

The coordinate $V_{j}$ is identified with an element of the space $\mathcal{V}=\mathbb{R}^{3}$, which is independent of $\Lambda$. The remainder coordinate $K_{j}$, however, is a map $K_{j}: \mathcal{P}_{j}(\Lambda) \rightarrow \mathcal{N}(\Lambda)$ and therefore does depend on $\Lambda$. In this section, we discuss the definition of $K_{j}$ in more detail, summarising full details given in [24]. Of particular importance are locality properties of $K_{j}$ which allow for the definition of a natural limiting space as $\Lambda_{N} \uparrow \mathbb{Z}^{d}$. We require some understanding of this infinite volume limit. In Section 8.1, we use results about the infinite volume limit to obtain estimates for finite $\Lambda_{N}$.

To discuss both the finite and infinite volume simultaneously, let $\mathbb{V}$ denote either $\Lambda_{N}$ or $\mathbb{Z}^{d}$, and set $N\left(\Lambda_{N}\right)=N$ and $N\left(\mathbb{Z}^{d}\right)=\infty$. We interpret the inequality $j \leq N(\mathbb{V})$ to mean that $j<\infty$ if $N(\mathbb{V})=\infty$. Given a finite set $X \subset \mathbb{V}$, recall that $\mathcal{N}(X)$ denotes the space of even differential forms defined in Section 3.1. We identify $\mathcal{N}(X)$ as a subalgebra of $\mathcal{N}(Y)$ if $X \subset Y$. We define $\mathcal{N}(\mathbb{V})$ to be the union over $\mathcal{N}(X)$ for all finite subsets $X \subset \mathbb{V}$. As in [24, Definition 1.4], we say that a polymer $X \in \mathcal{P}_{j}(\mathbb{V})$ is a small set if it is connected and if $|X|_{j}$, the number of $j$-blocks it 
contains, satisfies $|X|_{j} \leq 2^{d}$. We write $\mathcal{S}_{j}$ for the set of all small sets in $\mathcal{P}_{j}(\mathbb{V})$. Given any polymer $X \in \mathcal{P}_{j}(\mathbb{V})$, we define its small set neighbourhood $X^{\square}$ by

$$
X^{\square}=\bigcup_{Y \in \mathcal{S}_{j}: X \cap Y \neq \varnothing} Y .
$$

We write $\operatorname{Comp}_{j}(X) \subset \mathcal{P}_{j}(\mathbb{V})$ for the set of connected components of $X \in \mathcal{P}_{j}(\mathbb{V})$.

From [24, Definition 1.7], we recall the definition of the space $\mathcal{K}_{j}(\mathbb{V})$, as follows. The nonperturbative coordinate $K_{j}$ is an element of the space $\mathcal{K}_{j}(\Lambda)$.

Definition 6.2. For $j \leq N(\mathbb{V})$, let $\mathcal{K}_{j}=\mathcal{K}_{j}(\mathbb{V})$ be the real vector space of functions $K: \mathcal{P}_{j}(\mathbb{V}) \rightarrow$ $\mathcal{N}(\mathbb{V})$ with the properties,

- Field locality: $K(X) \in \mathcal{N}\left(X^{\square}, \mathbb{V}\right)$ for each connected $X \in \mathcal{P}_{j}$,

- Symmetry: $K$ is supersymmetric and Euclidean invariant,

- Component Factorisation: $K(X)=\prod_{Y \in \operatorname{Comp}_{j}(X)} K(Y)$ for all $X \in \mathcal{P}_{j}$.

The symmetries mentioned in Definition 6.2 are discussed above [24, Definition 1.7], and also in [6, 22, 23]. They do not play an explicit role in this paper, but they are needed in results we use from [6, 22 24]. We do not discuss them further here.

The analysis of [6,22 24] permits the inclusion of observables which break certain symmetries and are employed in [5,60] to study the decay of critical correlation functions. Observables are not needed in the present paper. We only need the case $\sigma=\bar{\sigma}=0$ of results in [6, 22, 24, we identify $K$ and $\pi_{\varnothing} K$, with $\pi_{*} K=0$ in the notation of those papers, and make no further references to observables here. In particular, Definition 6.2 has been specialised to the case where observables are not present.

\subsection{Norms}

For the analysis of $K_{j}$, we employ a family of norms on the spaces $\mathcal{K}_{j}(\mathbb{V})$, both for $\mathbb{V}=\Lambda_{N}$ and $\mathbb{V}=\mathbb{Z}^{d}$. These norms, which are defined in $[24,(1.45)]$, give rise to Banach spaces $\mathcal{W}_{j}(\tilde{s}, \mathbb{V}) \subset$ $\mathcal{K}_{j}(\mathbb{V})$ indexed by a parameter $\tilde{s}=\left(\tilde{m}^{2}, \tilde{g}\right) \in[0, \delta) \times(0, \delta)$ with fixed small $\delta>0$. The parameter $\tilde{s}$ is chosen depending on the mass $m^{2}$ and on $g_{0}$. The use of $\tilde{m}^{2}$ allows us the option to choose the norm parameter close to but not necessarily equal to the mass $m^{2}$ of the covariance $\left(-\Delta+m^{2}\right)^{-1}$, which permits variation of $m^{2}$ without changing the norm. The choice of $\tilde{g}$ is discussed below.

In the present paper, we do not need and therefore do not recall the definition of the norm from [24, (1.45)], needed for the propagation of estimates of $K$ from one scale to the next. We provide some general indication of the origin of these norms in the following discussion, with reference to [21,23, 24] for further details.

We discuss the norms for the case $\mathbb{V}=\Lambda$, the extension to $\mathbb{V}=\mathbb{Z}^{d}$ is discussed in [24]. Consider first a single differential form $F \in \mathcal{N}(\Lambda)$ which, as in Section 3.2, we regard as a function of the boson field $\phi$ and the fermion field $\psi$. For example, the degree-0 part $F^{0}$ is a function $F^{0}: \mathbb{C}^{\Lambda} \rightarrow \Lambda$ of $\phi$, in the ordinary sense. The degree-1 part of $F$ can be written as $\sum_{x \in \Lambda} F_{x}(\phi) \psi_{x}+\sum_{\bar{x} \in \Lambda} F_{\bar{x}}(\phi) \bar{\psi}_{\bar{x}}$, and each term can be viewed as an ordinary function of $\phi$ times a monomial of degree 1 in $\psi$. The coefficient $F_{x}(\phi)$ has the interpretation of a derivative of $F$ with respect to $\psi_{x}$ evaluated at $\psi=0$. 
The semi-norm $\|F\|_{T_{0, j}}$ provides a means to measure the size of these derivatives of $F$ with respect to $\phi$ and $\psi$ at $\phi=0$. Definitions of these and other norms, and development of their properties, is given in [21].

To prove Theorem 1.1, we only make use of the specialised set-up of [23, Section 1.1.6], omitting the parts that concern observables. In particular, we consider four copies of $\Lambda$, denoted $\Lambda_{b}, \bar{\Lambda}_{b}$, $\Lambda_{f}$, and $\bar{\Lambda}_{f}$, corresponding to the components of $\phi, \bar{\phi}, \psi, \bar{\psi}$, respectively, and set $\Lambda_{b}=\Lambda_{b} \sqcup \bar{\Lambda}_{b}$, $\Lambda_{f}=\Lambda_{f} \sqcup \bar{\Lambda}_{f}, \Lambda=\Lambda_{b} \sqcup \Lambda_{f}$, and we let $\vec{\Lambda}^{*}$ denote the set of finite ordered sequences of $\boldsymbol{\Lambda}$. A test function is a function $g: \vec{\Lambda}^{*} \rightarrow \mathbb{R}$.

In [23, Section 1.1.6] (see also [21, Section 3.3]), we define linear spaces of test functions $\Phi_{j}=\Phi_{j}\left(\ell_{j}\right)$. We set $\ell_{j}=\ell_{0} L^{-j[\phi]}$ for an appropriate constant $\ell_{0}$ (large and $L$-dependent), fix an integer $p_{\Phi} \geq \frac{1}{2} d+2$, and fix an integer $p_{\mathcal{N}} \geq 10$. The $\Phi_{j}\left(\ell_{j}\right)$ norm of a test function $g$ is defined by

$$
\|g\|_{\Phi_{j}\left(\ell_{j}\right)}=\sup _{z \in \vec{\Lambda}^{*}: p_{b}(z) \leq p_{\mathcal{N}} \alpha:|\alpha| \leq p_{\Phi}} \sup _{j}^{-z} L^{j|\alpha|}\left|\nabla^{\alpha} g_{z}\right|
$$

where $p_{b}(z) \leq p_{\mathcal{N}}$ means that $z$ has at most $p_{\mathcal{N}}$ boson components, $\ell_{j}^{-z}$ denotes $\ell_{j}^{-q}$ when $z=$ $\left(z_{1}, \ldots, z_{q}\right)$ with each $z_{i}$ in a copy of $\Lambda$, and the restriction $|\alpha| \leq p_{\Phi}$ limits the number of finitedifference derivatives to be at most $p_{\Phi}$ in each of the $q$ components of $z$. The number of fermion components of $z$ is unrestricted. For example, for a test function $\phi: \boldsymbol{\Lambda}_{b} \rightarrow \mathbb{R}$ with only one boson component,

$$
\ell_{0}^{-1} L^{-j[\phi]} L^{j|\alpha|_{1}}\left|\nabla^{\alpha} \phi_{x}\right| \leq\|\phi\|_{\Phi_{j}}
$$

for any multi-index $\alpha$ such that $|\alpha|_{1} \leq p_{\Phi}$. According to the discussion in Section 5.2, the fluctuation field at scale $j$, i.e., the Gaussian field with covariance $C_{j}$, when regarded as a test function in $\Phi_{j}\left(\ell_{j}\right)$ thus typically has norm $O(1)$. The scaling used in the definition of the norm has been designed to make this happen.

In [21], a pairing between forms and test functions is defined by

$$
\langle F, g\rangle_{0}=\sum_{z \in \vec{\Lambda}^{*}: p_{b}(z) \leq p_{\mathcal{N}}} \frac{1}{z !} F_{z}(0) g_{z} .
$$

The pairing can be thought of as a kind of generalised Taylor expansion, to all orders in the fermion field, and to order $p_{\mathcal{N}}$ in the boson field. We define a semi-norm on $\mathcal{N}(\Lambda)$ by

$$
\|F\|_{T_{0, j}}=\|F\|_{T_{0, j}\left(\ell_{j}\right)}=\sup _{g \in \Phi_{j}:\|g\|_{\Phi_{j}} \leq 1}\left|\langle F, g\rangle_{0}\right|
$$

The pairing of $F$ with test functions $g$ of norm 1 is intended to mimic how large $F$ should be when evaluated at typical small fields $(\bar{\phi}, \phi, \bar{\psi}, \psi)$, whose size is dictated by the scale- $j$ covariance.

It is also essential to have some control over large fields; this issue is addressed in detail in [23]. As motivation, consider the probability measure on $\mathbb{R}$ given by

$$
\mu(d s) \propto e^{-g s^{4}} d s,
$$

which has mean 0 and variance proportional to $g^{-1 / 2}$. More informally, typically $|s|=O\left(g^{-1 / 4}\right)$. The expectation $\mathbb{E}_{j+1} \theta\left(I_{j} \circ K_{j}(\Lambda)\right)$ contains a factor $e^{-g_{j}|\phi|^{4}}$ which suggests that typically $|\phi|=$ $O\left(g_{j}^{-1 / 4}\right)$. Since we do not know a priori the value of $g_{j}$, we design the norm $\left\|K_{j}\right\|_{\mathcal{W}_{j}(\tilde{s}, \mathbb{V})}$ so that, 
in addition to taking small fields into account, it also measures the size of $K_{j}$ when evaluated on fields of size $|\phi|=O\left(\tilde{g}_{j}^{-1 / 4}\right)$ with $\tilde{g}_{j}$ an approximate guess for $g_{j}$. We then show a posteriori that the guess does what is required. The sequence $\bar{g}_{j}=\bar{g}_{j}\left(m^{2}, g_{0}\right)$ determined by $\bar{g}_{0}=g_{0}$ and the recursion (6.2) is a natural candidate for $\tilde{g}_{j}$, but is one that introduces dependence of the norms on the two parameters $m^{2}$ and $g_{0}$. The dependence on $g_{0}$ cannot be avoided, but it is convenient to use a family of norms that minimises the dependence on $m^{2}$. To accomplish this, we set

$$
\tilde{g}_{j}\left(m^{2}, g_{0}\right)=\bar{g}_{j}\left(0, g_{0}\right) \mathbb{1}_{j \leq j_{m}}+\bar{g}_{j_{m}}\left(0, g_{0}\right) \mathbb{1}_{j>j_{m}},
$$

where the mass scale $j_{m}$ is the smallest integer $j$ such that $L^{2 j} m^{2} \geq 1$. Thus $\tilde{g}_{j}\left(m^{2}, g_{0}\right)$ is the massless sequence $\bar{g}_{j}\left(0, g_{0}\right)$ with its evolution shut down at the mass scale. We show in Lemma 7.4 that $\tilde{g}_{j}\left(m^{2}, g_{0}\right)=\bar{g}_{j}\left(m^{2}, g_{0}\right)+O\left(\bar{g}_{j}^{2}\left(m^{2}, g_{0}\right)\right)$, so the sequences $\tilde{g}_{j}$ and $\bar{g}_{j}$ are close to being equal.

A property of the norms that we use in the proof of Theorem 4.1 (see Section 8.4) is the fact given by $[24,(1.46)]$ that the $\mathcal{W}_{N}$ norm dominates the $T_{0, N}$ semi-norm, in the sense that

$$
\|F(\Lambda)\|_{T_{0, N}} \leq\|F\|_{\mathcal{W}_{N}(\tilde{s}, \Lambda)}
$$

\subsection{Specification of renormalisation group map}

For both choices $\mathbb{V}=\Lambda_{N}$ and $\mathbb{V}=\mathbb{Z}^{d}$, explicit definitions of maps (5.29) are constructed in [24]. For $\mathbb{V}=\Lambda$, the maps achieve the objective (5.30). The significance for $\mathbb{V}=\mathbb{Z}^{d}$ is discussed in Section 6.5 below. To simplify the notation, we write (5.29) as $(V, K) \mapsto\left(V_{+}, K_{+}\right)$, typically dropping subscripts $j$ and writing + in place of $j+1$, and also leaving the dependence of the maps on the mass parameter $m^{2}$ implicit. As discussed in Section 6.3, it is necessary to make assumptions on $(V, K)$, in order for these definitions to be well-defined and to obtain useful estimates for $\left(V_{+}, K_{+}\right)$. These assumptions are stated in terms of domains, defined as follows.

Given $C_{\mathcal{D}}>1, \alpha>0, \delta>0$, and $\tilde{s}=\left(\tilde{m}^{2}, \tilde{g}\right) \in[0, \delta) \times(0, \delta)$, let

$$
\mathbb{D}_{j}(\tilde{s}, \mathbb{V})=\left\{(g, z, \nu): C_{\mathcal{D}}^{-1} \tilde{g}<g<C_{\mathcal{D}} \tilde{g},|z|,|\mu|<C_{\mathcal{D}} \tilde{g}\right\} \times B_{\mathcal{W}_{j}(\tilde{s}, \mathbb{V})}\left(\alpha \tilde{\chi}_{j} \tilde{g}^{3}\right),
$$

where $B_{X}(r)$ is the open ball of radius $r$ around the origin in the Banach space $X$, and $\tilde{\chi}_{j}=\chi_{j}\left(\tilde{m}^{2}\right)$ with $\chi_{j}$ defined by (6.7). Roughly speaking, the domain (6.17) permits small $g>0$ that is bounded away from zero, with $z, \mu=O(g)$, and with $K$ bounded in a precise but non-trivial fashion by $O\left(g^{3}\right)$. The domain $\mathbb{D}_{j}(\tilde{s}, \mathbb{V})$ is equipped with the norm of $\mathcal{V} \times \mathcal{W}_{j}(\tilde{s}, \mathbb{V})$.

The parameter $\tilde{m}^{2}$ that appears in the definition of the domain (6.17) would ideally be set equal to the mass $m^{2}$ appearing in the covariance, but this would be problematic for the discussion of continuity of the renormalisation group map as a function of $m^{2}$. Thus we decouple $m^{2}$ from the domain by using $\tilde{m}^{2}$ in the domain and requiring $m^{2}$ to be close to but not necessarily equal to $\tilde{m}^{2}$. For this, we recall the interval $\mathbb{I}_{j}$ defined in (5.12), and set

$$
\tilde{\mathbb{I}}_{j}=\tilde{\mathbb{I}}_{j}\left(\tilde{m}^{2}\right)= \begin{cases}{\left[\frac{1}{2} \tilde{m}^{2}, 2 \tilde{m}^{2}\right] \cap \mathbb{I}_{j}} & \left(\tilde{m}^{2} \neq 0\right) \\ {\left[0, L^{-2(j-1)}\right] \cap \mathbb{I}_{j}} & \left(\tilde{m}^{2}=0\right) .\end{cases}
$$

For $j<N$, these intervals are illustrated in Figure 2 .

For $\tilde{s}=\left(\tilde{m}^{2}, \tilde{g}\right) \in[0, \delta) \times(0, \delta)^{2}$, the maps $V_{+}=V_{+, \mathbb{V}}$ and $K_{+}=K_{+, \mathbb{V}}$ with mass $m^{2} \in \tilde{\mathbb{I}}_{+}\left(\tilde{m}^{2}\right)$ are maps

$$
V_{+}: \mathbb{D}(\tilde{s}, \mathbb{V}) \rightarrow \mathcal{V}, \quad K_{+}: \mathbb{D}(\tilde{s}, \mathbb{V}) \rightarrow \mathcal{K}_{+}(\mathbb{V})
$$




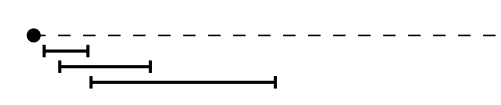

Figure 2: The dashed line illustrates the interval $\mathbb{I}_{j}=[0, \delta)$ for $j<N$. The intervals $\tilde{\mathbb{I}}_{j}\left(\tilde{m}^{2}\right)$ are given by $\left[\frac{1}{2} \tilde{m}^{2}, 2 \tilde{m}^{2}\right]$ if $\tilde{m}^{2} \in\left(0, \frac{1}{2} \delta\right)$ and are illustrated below the dashed line. As $\tilde{m}^{2} \downarrow 0$, the length of these intervals shrinks to 0 , but $\tilde{\mathbb{I}}_{j}(0)$ is given by the non-empty interval $\left[0, L^{-2(j-1)}\right]$.

such that, given $(V, K) \in \mathbb{D}_{j}(\tilde{s}, \Lambda)$,

$$
\mathbb{E}_{+} \theta(I(V) \circ K)(\Lambda)=\left(I_{+}\left(V_{+}(V, K)\right) \circ K_{+}(V, K)\right)(\Lambda)
$$

There is no analogue of (6.20) for the case $\mathbb{V}=\mathbb{Z}^{d}$, and we postpone discussion of infinite volume to Section 6.5. The renormalisation group map is defined by (6.19). As mentioned in Section 5.5. there are trivial choices of maps that make (6.20) hold. The maps of [24] provide a nontrivial and good choice, a choice which obeys the useful estimates discussed below.

In the present paper, we do not need or use the precise definitions of the maps $\left(V_{+}, K_{+}\right)$, so we discuss only their existence and certain of their important properties from [24]. Since the maps are given by explicit formulas, not depending on the parameter $\tilde{s}$ appearing their domains in (6.19), the maps viewed as maps on different domains coincide on their intersections, and we will apply this property without further comment.

The map $V_{+}$is a perturbation of the map $\varphi_{\mathrm{pt}}^{(0)}$ discussed in Section 6.1, and it is convenient to describe it in terms of the difference

$$
R_{+}(V, K)=V_{+}(V, K)-\varphi_{\mathrm{pt}}^{(0)}(V)
$$

The following theorem, which is illustrated by Figure 3 , provides estimates for the maps $R_{+}, K_{+}$, combining [24, Theorems 1.10 1.11, 1.13] into a single statement. In particular, we apply the result of [24, Theorem 1.10] in the form given in [24, (1.61)]. For the statement of the theorem, we view $R_{+}, K_{+}$as maps jointly on $\left(V, K, m^{2}\right) \in \mathbb{D}(\tilde{s}) \times \tilde{\mathbb{I}}_{+}\left(\tilde{m}^{2}\right)$. The $L^{p, q}$ norm is the operator norm of a multi-linear operator from $\mathcal{V}^{p} \times \mathcal{W}^{q}$ to $\mathcal{V}$ or to $\mathcal{W}_{+}$, for $R_{+}$or $K_{+}$, respectively, and $\tilde{\chi}=\chi_{j}\left(\tilde{m}^{2}\right)$ where $\chi_{j}$ is given by (6.7). We have replaced occurrences of $\chi^{3 / 2}$ and $\chi^{1 / 2}$ from [24] by $\chi$ here; this amounts to an unimportant redefinition of the parameter $\Omega$. Also, in (6.22), $\tilde{s}_{+}=\left(\tilde{m}^{2}, \tilde{g}_{+}\right)$ for any choice of $\tilde{g}_{+} \in\left[\frac{1}{2} \tilde{g}, 2 \tilde{g}\right]$ (as required by [24, (1.30)]). It is straightforward to verify that the sequence defined by (6.15) obeys this constraint. For application of a single renormalisation group step in Theorem 6.3, there is considerable flexibility in the definition of $\tilde{g}$. However, indefinite iteration of the renormalisation group map requires a careful choice of the sequence $\tilde{g}_{j}$, and (6.15) provides a choice that works.

Theorem 6.3. Let $d=4$ and $\mathbb{V}=\Lambda_{N}$. Let $C_{\mathcal{D}}$ and $L$ be sufficiently large, and let $p, q \in \mathbb{N}_{0}$. There exist $M>0$ (depending on $p, q$ ), $\delta>0$, and $\kappa=O\left(L^{-1}\right)$ such that for $\tilde{g} \in(0, \delta)$ and $\tilde{m}^{2} \in \mathbb{I}_{+}$, and with the domain $\mathbb{D}$ defined using any $\alpha>M$, the maps

$$
R_{+}: \mathbb{D}(\tilde{s}, \Lambda) \times \tilde{\mathbb{I}}_{+}\left(\tilde{m}^{2}\right) \rightarrow \mathcal{V}, \quad K_{+}: \mathbb{D}(\tilde{s}, \Lambda) \times \tilde{\mathbb{I}}_{+}\left(\tilde{m}^{2}\right) \rightarrow \mathcal{W}_{+}\left(\tilde{s}_{+}, \Lambda\right)
$$




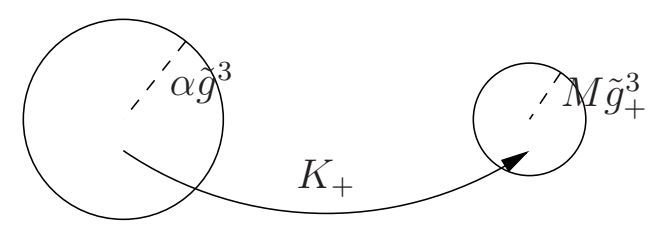

Figure 3: The map $K_{+}$of Theorem 6.3 maps the centred ball of radius $\alpha \tilde{g}^{3}$ to a ball of smaller radius $M \tilde{g}_{+}^{3}$ (in our application $\alpha=4 M$ ).

are analytic in $(V, K)$, and satisfy the estimates

$$
\begin{gathered}
\left\|D_{V}^{p} D_{K}^{q} R_{+}\right\|_{L^{p, q}} \leq \begin{cases}M \tilde{\chi} \tilde{g}_{+}^{3-p} & (p \geq 0, q=0) \\
M \tilde{g}_{+}^{1-p-q} & (p \geq 0, q=1,2) \\
0 & (p \geq 0, q \geq 3)\end{cases} \\
\left\|D_{V}^{p} D_{K}^{q} K_{+}\right\|_{L^{p, q}} \leq \begin{cases}M \tilde{\chi} \tilde{g}_{+}^{3-p} & (p \geq 0) \\
\kappa & (p=0, q=1) \\
M \tilde{g}_{+}^{-p}\left(\tilde{\chi} \tilde{g}_{+}^{10 / 4}\right)^{1-q} & (p \geq 0, q \geq 1) .\end{cases}
\end{gathered}
$$

In addition, $R_{+}, K_{+}$, and every Fréchet derivative in $(V, K)$, when applied as a multilinear map to directions $\dot{V}$ in $\mathcal{V}^{p}$ and $\dot{K}$ in $\mathcal{W}^{q}$, is jointly continuous in all arguments, $m^{2}, V, K, \dot{V}, \dot{K}$.

We emphasise that when $\tilde{m}^{2}=0$ in Theorem 6.3, the continuity statement includes rightcontinuity at $m^{2}=0$. The bounds for $(p, q)=(0,0)$ show that $R_{+}$and $K_{+}$are third-order in $\tilde{g}$ and thus third-order in $V$. The $(p, q)=(0,1)$ bound of (6.24) shows that the $K$-coordinate is contracting, since $\kappa<1$. Thus, viewed as a dynamical system, $(V, K) \mapsto\left(V_{+}, K_{+}\right)$has two centre directions $g$ and $z$, one expanding direction $\mu$, and an infinite-dimensional contracting direction $K$. Theorem 6.3 is the deepest ingredient in our analysis; its proof occupies most of [23, 24]. The development of the norms defined in [21] culminates in the $\mathcal{W}$ norm needed for Theorem 6.3, and the operator Loc defined in [22] is designed to achieve the contraction in $K$.

\subsection{Renormalisation group map in infinite volume}

In our context, the renormalisation group map is most naturally defined to be a map in the setting of a torus, since a defining property is that it should preserve the circle product under expectation as in (6.20). We have no analogue of (6.20) for the infinite volume $\mathbb{Z}^{d}$, for which we have defined neither the expectation nor the circle product. Nevertheless, there is a natural definition of a map $(V, K) \mapsto\left(V_{+}, K_{+}\right)$which is set on $\mathbb{Z}^{d}$ rather than on a torus $\Lambda$, as an appropriate inductive limit of the corresponding maps on the family of all tori. The infinite volume map has the advantage that it is defined for all scales $j \in \mathbb{N}$, with no restriction due to finite volume. In particular, we can study the limit $j \rightarrow \infty$, which we use in an important way to apply the main result of [7] to the dynamical system defined by the renormalisation group.

The definition of the renormalisation group map on $\mathbb{Z}^{d}$ is discussed in detail in [24, Section 1.8.3]. There a correspondence is established between elements of the family of spaces $\mathcal{K}_{j}(\Lambda)$ indexed by $\Lambda$ which obey a compatibility property called Property $\left(\mathbb{Z}^{d}\right)$, and the space $\mathcal{K}_{j}\left(\mathbb{Z}^{d}\right)$. In brief, given 
$X \in \mathcal{P}_{j}\left(\mathbb{Z}^{d}\right)$ and any $\Lambda$ whose period is at least twice the diameter of $X$, there is a map $\iota: X \rightarrow \Lambda$ such that nearest neighbours in $X$ are mapped to nearest neighbours in $\Lambda$ and the preimages of nearest neighbours in $\Lambda$ are nearest neighbours in $X$. The above-mentioned correspondence permits identification of $K_{j, \Lambda}(\iota X)$ with $K_{j, \mathbb{Z}^{d}}(X)$ for all $\Lambda$ whose period is at least twice the diameter of $X$. The diameter restriction causes information to be lost in going from the finite volume $K$ to its infinite volume version, as there is no infinite volume counterpart to $K(U) \in \mathcal{K}(\Lambda)$ whenever $U$ is comparable in size to $\Lambda$, and in particular if $U$ "wraps around" the torus $\Lambda$. This does not cause difficulties since our analysis shows that the scale- $j$ norm of $K(U)$ decays exponentially in the number of scale- $j$ blocks in $U$, and the significant information is maintained by the correspondence.

It is shown in [24, Proposition 1.17] that

$$
\text { if the family }\left(K_{\Lambda}\right) \text { has Property }\left(\mathbb{Z}^{d}\right) \text { then so does the family }\left(K_{+, \Lambda}\right) \text {, }
$$

and this permits the definition of a map $\left(V, K_{\mathbb{Z}^{d}}\right) \mapsto K_{+, \mathbb{Z}^{d}}$. Moreover, it is shown in [24, Proposition 1.18 that the map $V_{+}$can be regarded as a map on $\left(V, K_{\mathbb{Z}^{d}}\right) \in \mathbb{D}\left(\mathbb{Z}^{d}\right)$, and in addition

if the family $\left(K_{\Lambda}\right)$ has Property $\left(\mathbb{Z}^{d}\right)$ then $V_{+, \Lambda}\left(V, K_{\Lambda}\right)=V_{+, \mathbb{Z}^{d}}\left(V, K_{\mathbb{Z}^{d}}\right)$ for all $\Lambda$.

In particular, this shows that the loss of information in going from $\left(K_{\Lambda}\right)$ to $K_{\mathbb{Z}^{d}}$ does not affect the flow of coupling constants.

The following theorem is proved in [24, Theorem 1.19]. In particular, it shows that $R_{+, \mathbb{Z}^{d}}$ and $K_{+, \mathbb{Z}^{d}}$ (in the space $\left.\mathcal{W}(0, \tilde{g})\right)$ are right-continuous at $m^{2}=0$ for all $j<\infty$.

Theorem 6.4. All statements of Theorem 6.3 hold, including domains and estimates, with the same parameters and constants, when $\mathbb{V}=\Lambda$ is replaced by $\mathbb{V}=\mathbb{Z}^{d}$.

\subsection{Renormalisation group map in transformed variables}

In Section 6.1. we introduced the transformation $T_{j}$, and discussed its effect on the perturbative quadratic part of the renormalisation group flow. We now show that its effect on the nonperturbative part is insignificant. The transformed renormalisation group flow is important in proofs in Sections 7 , 8 .

We distinguish versions of the renormalisation group coordinates and maps in transformed variables by writing, e.g., $\check{V}=T(V)$, and $\left(\check{V}_{+}, \check{K}_{+}\right)$for versions of the maps $\left(V_{+}, K_{+}\right)$which act on transformed variables $(\breve{V}, K)$. Since the transformation acts trivially for $j=0$, i.e., $T_{0}(V)=V$, we do not distinguish between $V_{0}$ and $\check{V}_{0}$ and use both interchangeably. In more detail, the maps $\check{V}_{+}, \check{K}_{+}$are defined by

$$
\check{V}_{+}(\check{V}, K)=T_{+}\left(V_{+}\left(T^{-1}(\check{V}), K\right)\right), \quad \check{K}_{+}(\check{V}, K)=K_{+}\left(T^{-1}(\check{V}), K\right),
$$

and we also set

$$
\check{R}_{+}(\check{V}, K)=\check{V}_{+}(\check{V}, K)-\bar{\varphi}(\check{V}) .
$$

As in Section 6.4, dependence on $m^{2}$ is left implicit in the notation, and the subscript + is shorthand for scale $j+1$.

We extend $T, T^{-1}$ to act trivially on $K$, i.e., $T(V, K)=(T(V), K), T^{-1}(V, K)=\left(T^{-1}(V), K\right)$, and set $\check{\mathbb{D}}=T(\mathbb{D})$. Since $T$ is invertible for $V$ sufficiently small (which we assume), also $T^{-1}(\check{\mathbb{D}})=$ $\mathbb{D}$. 
Corollary 6.5. All statements of Theorems 6.36.4 (namely regularity, domains, estimates) hold when $R_{+}, K_{+}$are replaced by $\check{R}_{+}, \check{K}_{+}$.

Proof. We first note that since $T$ is a polynomial with $T(V)=V+O\left(\|V\|^{2}\right)$, for bounded $p$,

$$
\left\|D^{p} T(V)\right\| \leq O(1), \quad\left\|D^{p} T^{-1}(V)\right\| \leq O(1) .
$$

The chain rule and (6.29) imply

$$
\left\|D_{\check{V}}^{p} D_{K}^{q} \check{K}_{+}(\check{V}, K)\right\| \leq C \sum_{k=1}^{p}\left\|D_{V}^{k} D_{K}^{q} K_{+}\left(T^{-1}(\check{V}), K\right)\right\|,
$$

and the claim for $\check{K}_{+}$immediately follows from (6.24) since the bound on the deriviative of $K_{+}$is largest for $k=p$.

To bound the derivatives of $\check{R}_{+}$, we write $\check{R}_{+}=\check{R}_{\mathrm{pt}}+\check{R}_{*}$, where

$$
\begin{aligned}
\check{R}_{\mathrm{pt}}(\check{V}) & =T_{+}\left(\varphi_{\mathrm{pt}}^{(0)}\left(T^{-1}(\check{V})\right)\right)-\bar{\varphi}(\check{V}) \\
\check{R}_{*}(\check{V}, K) & \left.=T_{+}\left(\varphi_{\mathrm{pt}}^{(0)}\left(T^{-1}(\check{V})\right)+R_{+}\left(T^{-1}(\check{V}), K\right)\right)-T_{+}\left(\varphi_{\mathrm{pt}}^{(0)}\left(T^{-1}(\check{V})\right)\right)\right) .
\end{aligned}
$$

It suffices to show that $\check{R}_{\mathrm{pt}}, \check{R}_{*}$ both satisfy the bounds claimed for $R_{+}$in (6.23). As discussed below (6.1),$T_{+} \circ \varphi_{\mathrm{pt}}^{(0)} \circ T^{-1}=\bar{\varphi}+O\left(\|V\|^{3}\right)$. Since $\varphi_{\mathrm{pt}}^{(0)}, \bar{\varphi}, T, T_{+}$are polynomials, it follows that

$$
D_{\check{V}}^{p}\left(T_{+} \circ \varphi_{\mathrm{pt}}^{(0)} \circ T^{-1}\right)=D_{\check{V}}^{p} \bar{\varphi}+O\left(\|\check{V}\|^{3-p}\right) .
$$

In particular, since $\check{R}_{\mathrm{pt}}$ is independent of $K$, this shows that $\check{R}_{\mathrm{pt}}$ satisfies (6.23). For $\check{R}_{*}$, we write the quadratic polynomial $T_{+}$as $T_{+}(V)=V+B_{+}(V, V)$ with $B_{+}\left(V_{1}, V_{2}\right)$ bilinear, and we set $f=\varphi_{\mathrm{pt}}^{(0)}\left(T^{-1}(\check{V})\right)$ and $r=R_{+}\left(T^{-1}(\check{V}), K\right)$. Then

$$
\check{R}_{*}=r+B_{+}(r, f)+B_{+}(f, r)+B_{+}(r, r) .
$$

The first term is bounded exactly as (6.30) and the others can be seen to be smaller in a similar way. This completes the proof.

\section{Renormalisation group flow}

We now discuss the global aspect of the renormalisation group: the enabling of indefinitely repeated application of the renormalisation group map, as the scale $j$ increases. It is this global aspect that requires the careful choice of $\left(\nu_{0}^{c}, z_{0}^{c}\right)$ in Theorem 4.1, and that leads to the identification of the critical point. We restrict throughout Section 7 to the infinite volume case, $\mathbb{V}=\mathbb{Z}^{d}$. In Section 8 , we return to the case $\mathbb{V}=\Lambda_{N}$. 


\subsection{Existence and regularity of global flow}

We say that $\left(V_{j}, K_{j}\right)_{j \in \mathbb{N}_{0}}$ is a global flow of the infinite volume renormalisation group if

$$
\left(V_{j+1}, K_{j+1}\right)=\left(V_{j+1}\left(V_{j}, K_{j}\right), K_{j+1}\left(V_{j}, K_{j}\right)\right) \quad \text { for all } j \in \mathbb{N}_{0} .
$$

In (7.1), on the left-hand side $\left(V_{j+1}, K_{j+1}\right)$ is an element in the sequence $\left(V_{j}, K_{j}\right)$, and on the right-hand side it denotes the map $\left(V_{+}, K_{+}\right)=\left(V_{j+1}, K_{j+1}\right)$ of Section [6; the interpretation should be clear from context. We suppress the dependence on the mass parameter $m^{2}$ in our notation.

The following proposition, which sits at the centre of our analysis, constructs a sequence with the desired properties. For its statement, we fix the parameter $\alpha$ in the definition of the domains $\mathbb{D}$ in (6.17) as $\alpha=4 M$, where $M$ is the constant in Theorem 6.3 (this choice is convenient, but somewhat arbitrary).

Proposition 7.1. Let $d=4$ and $\mathbb{V}=\mathbb{Z}^{d}$, and let $\delta>0$ be sufficiently small. There are continuous functions of $\left(m^{2}, g_{0}\right)$, namely $z_{0}^{c}, \mu_{0}^{c}:[0, \delta)^{2} \rightarrow \mathbb{R}$, with $z_{0}^{c}\left(m^{2}, 0\right)=\mu_{0}^{c}\left(m^{2}, 0\right)=0$, continuously differentiable in $g_{0} \in(0, \delta)$ with uniformly bounded derivatives, such that for all $\left(\mathrm{m}^{2}, g_{0}\right) \in[0, \delta) \times$ $(0, \delta)$, the global flow (7.1) with mass parameter $m^{2}$ and initial condition given by

$$
V_{0}=\left(g_{0}, z_{0}^{c}\left(m^{2}, g_{0}\right), \mu_{0}^{c}\left(m^{2}, g_{0}\right)\right), \quad K_{0}=\mathbb{1}_{\varnothing},
$$

exists, and $\left(V_{j}, K_{j}\right) \in \mathbb{D}_{j}\left(s_{j}, \mathbb{Z}^{d}\right)$ for all $j \in \mathbb{N}_{0}$. Here $s_{j}=\left(m^{2}, \tilde{g}_{j}\left(m^{2}, g_{0}\right)\right)$ with $\tilde{g}_{j}$ given by (6.15)). In particular, $\left\|K_{j}\right\|_{\mathcal{W}_{j}\left(s_{j}\right)}=O\left(\chi_{j} \bar{g}_{j}^{3}\right)$ and $\check{g}_{j}, g_{j}=O\left(\bar{g}_{j}\right)$. In addition, $\check{z}_{j}, \check{\mu}_{j}, z_{j}, \mu_{j}=O\left(\chi_{j} \bar{g}_{j}\right)$.

Proposition 7.1 can be extended to replace its initial condition $K_{0}=\mathbb{1}_{\varnothing}$ by a more general condition on $K_{0}$. The extension is not required for our present purposes, so is not pursued here, but a more general initial condition is needed for the analysis of weakly self-avoiding walk with nearest-neighbour attraction [4].

The proof of Proposition 7.1 is based an application of the main result of [7], which concerns a class of non-hyperbolic dynamical systems. For this, it is advantageous to work with the transformed variables $\check{V}_{j}=T_{j}\left(V_{j}\right)$ defined in (6.1)-(6.4), as this produces a triangular system to second order. We therefore reformulate the renormalisation group map $(V, K) \mapsto\left(V_{+, \mathbb{Z}^{d}}, K_{+, \mathbb{Z}^{d}}\right)$ of Section 6.5] in terms of the transformed variables. This was anticipated in Section 6.6, where maps $\check{V}_{+}, \check{K}_{+}, \check{R}_{+}$are defined. Thus we define the evolution map

$$
\begin{aligned}
\Phi_{j}\left(\check{V}_{j}, K_{j}\right) & =\left(\check{V}_{j+1}\left(\check{V}_{j}, K_{j}\right), \check{K}_{j+1}\left(\check{V}_{j}, K_{j}\right)\right) \\
& =\left(\bar{\varphi}_{j}\left(\check{V}_{j}\right)+\check{R}_{j+1}\left(\check{V}_{j}, K_{j}\right), \check{K}_{j+1}\left(\check{V}_{j}, K_{j}\right)\right) .
\end{aligned}
$$

The map $\bar{\varphi}$ is given by (6.2)-(6.4), and its global flow with initial condition $\bar{g}_{0}$ and final condition $\left(\bar{z}_{\infty}, \bar{\mu}_{\infty}\right)=(0,0)$ is determined by Proposition 6.1. Precise statements about the domains of the $\Phi_{j}$ are deferred to Section 7.3 .

We interpret $\Phi_{j}$ as the time-dependent evolution map of a dynamical system, which is an infinite-dimensional perturbation of the 3-dimensional dynamical system $\bar{\varphi}$ on $\mathcal{V}=\mathbb{R}^{3}$. The maps $\left(\Phi_{j}\right)$ are between different spaces, since $K_{j}$ and $K_{j+1}$ are functions of polymers defined in terms of blocks of different side lengths $L^{j}$ and $L^{j+1}$, respectively, but this aspect is unimportant. Although we have not defined $\left(V_{+}, K_{+}\right)$at $(V, K)=(0,0)$ in Section 6.4, it is natural to extend it to act trivially on $(0,0)$, which can thus be regarded a fixed point of the dynamical system $\Phi$. We are 
interested in the behaviour of $\Phi$ near this fixed point. In particular, we wish to construct a sequence $\left(\check{V}_{j}, K_{j}\right)$ that satisfies the flow equation

$$
\left(\check{V}_{j+1}, K_{j+1}\right)=\Phi_{j}\left(\check{V}_{j}, K_{j}\right)
$$

with good estimates on the sequence $\left(\check{V}_{j}, K_{j}\right)$ and with boundary conditions

$$
K_{0}=\mathbb{1}_{\varnothing}, \quad \check{g}_{0}>0 \text { small, } \quad \check{z}_{\infty}=\check{\mu}_{\infty}=0 .
$$

The condition $\check{g}_{0}>0$ is related to the stability problem discussed around (6.14); because of it the fixed point can be approached from one side only.

\subsection{Non-hyperbolic dynamical system}

The dynamical system (7.4) is not hyperbolic near the fixed point $(0,0)$, due to the two unit eigenvalues of $\bar{\varphi}$ corresponding to the variables $g, z$. To study it, we apply the main result of [7], which considers a general class of dynamical systems $\Phi=\left(\Phi_{j}\right)$ with non-hyperbolic fixed point and with contractive coordinate $K_{j}$ lying in a given sequence of Banach spaces $\mathcal{W}_{j}$. The result of [7] shows that, under appropriate assumptions on $\Phi_{j}$, there exists a global flow satisfying the boundary conditions (7.5). We now recall this result in the form we require. Because we apply the result to the renormalisation group flow in the transformed variables discussed in Section 6.1, we state it in the notation of transformed variables $(\check{g}, \check{z}, \check{\mu}, K)$ rather than the variables $(g, z, \mu, K)$ used in [7].

The dynamical system studied in [7] involves a quadratic flow map $\bar{\varphi}$, of which the flow defined by (6.2) -6.4) is an instance. This flow map in [7] is required to obey the Assumptions (A1-A2) stated in Section 6.1, with a fixed $\Omega>1$ which defines $j_{\Omega}$ as in (6.6). Any such quadratic flow has a unique global flow which obeys the boundary conditions of our interest, and this global flow obeys the estimates of Proposition 6.1; this is proved in [7, Proposition 1.2. We have already seen that the specific quadratic flow (6.2) - 6.4 obeys Assumptions (A1-A2) and Proposition 6.1. In this section, we consider any quadratic flow that obeys Assumptions (A1-A2), not necessarily our specific example (6.2)-(6.4), and we replace $\Phi$ of (17.3) by the more general map

$$
\Phi_{j}\left(\check{V}_{j}, K_{j}\right)=\left(\bar{\varphi}_{j}\left(\check{V}_{j}\right)+\rho_{j}\left(\check{V}_{j}, K_{j}\right), \psi_{j}\left(\check{V}_{j}, K_{j}\right)\right),
$$

where $\left(\rho_{j}, \psi_{j}\right)$ satisfy Assumption (A3) stated below.

To formulate the theorem, let $\mathcal{V}=\mathbb{R}^{3}$, let $\left(\mathcal{W}_{j}\right)_{j \in \mathbb{N}_{0}}$ be a sequence of Banach spaces, and set $X_{j}=\mathcal{V} \oplus \mathcal{W}_{j}$ (compared with [7] we have reversed the order of the components $\mathcal{V}, \mathcal{W}_{j}$ ). We define domains $D_{j} \subset X_{j}$ on which $\left(\rho_{j}, \psi_{j}\right)$ is assumed to be defined, and an assumption which states estimates for $\left(\rho_{j}, \psi_{j}\right)$, as follows. The domain and estimates depend on the initial condition $g_{0}$ and an external parameter $m^{2}$ (here we write $m^{2}$ instead of $m$ as in [7]). For parameters $a, h>0$ and sufficiently small $g_{0}>0$, let $\left(\bar{g}_{j}, \bar{z}_{j}, \bar{\mu}_{j}\right)_{j \in \mathbb{N}_{0}}$ be the sequence determined by Proposition 6.1 with initial condition $\bar{g}_{0}=g_{0}$ and mass $m^{2}$, and define the domain $D_{j}=D_{j}\left(m^{2}, g_{0}, a, h\right) \subset X_{j}$ by

$$
\begin{aligned}
& D_{j}=\left\{x_{j}=\left(\check{g}_{j}, \check{z}_{j}, \check{\mu}_{j}, K_{j}\right) \in X_{j}:\left|\check{g}_{j}-\bar{g}_{j}\right| \leq h \bar{g}_{j}^{2}\left|\log \bar{g}_{j}\right|,\right. \\
& \left|\check{z}_{j}-\bar{z}_{j}\right| \leq h \chi_{j} \bar{g}_{j}^{2}\left|\log \bar{g}_{j}\right| \text {, } \\
& \left|\check{\mu}_{j}-\bar{\mu}_{j}\right| \leq h \chi_{j} \bar{g}_{j}^{2}\left|\log \bar{g}_{j}\right| \text {, } \\
& \left.\left\|K_{j}\right\|_{\mathcal{W}_{j}} \leq a \chi_{j} \bar{g}_{j}^{3}\right\} \text {. }
\end{aligned}
$$




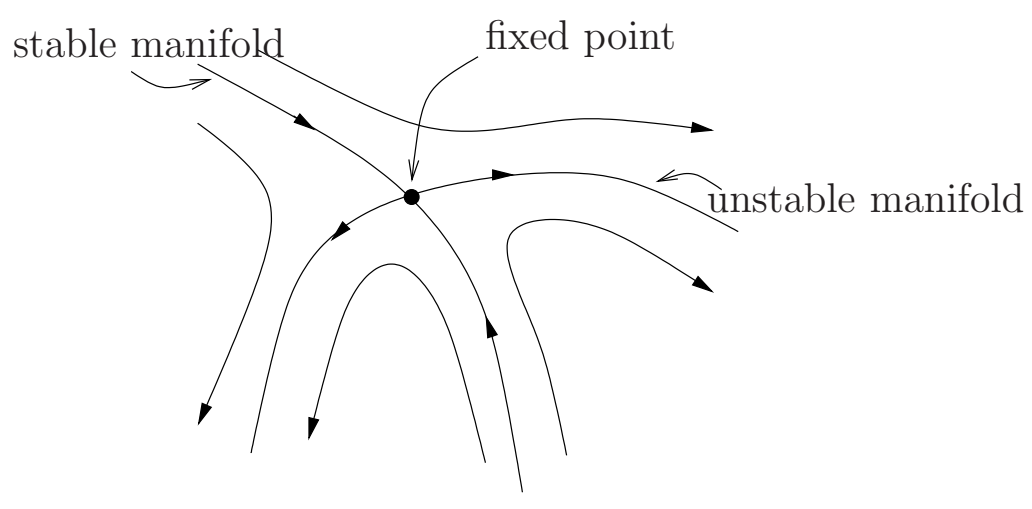

Figure 4: Schematic phase portrait of the global flow of Theorem 7.2. In the renormalisation group flow of Proposition 7.1, the portion of the stable manifold near the fixed point $(V=(0,0,0), K=0)$ restricted to $K=0$ consists of the points $V=\left(g_{0}, \mu_{0}^{c}\left(g_{0}\right), z_{0}^{c}\left(g_{0}\right)\right), g_{0} \in[0, \delta]$.

Assumptions (A1-A2) for the quadratic flow are supplemented with the following assumption for the perturbation $\left(\rho_{j}, \psi_{j}\right)$. Constants in Assumption (A3) carry subscripts "3" to indicate that they arise in (A3).

Assumption (A3). The perturbation: The maps $\rho_{j}: D_{j} \rightarrow \mathcal{V} \subset X_{j+1}$ and $\psi_{j}: D_{j} \rightarrow \mathcal{W}_{j+1} \subset$ $X_{j+1}$ are three times continuously Fréchet differentiable, and there exist $\kappa \in\left(0, \Omega^{-1}\right), R \in(0, a(1-$ $\kappa \Omega)), M>0$ such that, for all $x_{j}=\left(V_{j}, K_{j}\right) \in D_{j}$,

$$
\begin{gathered}
\left\|D_{V}^{p} D_{K}^{q} \rho_{j}\left(x_{j}\right)\right\|_{L^{p, q}} \leq \begin{cases}M \chi_{j+1} \bar{g}_{j+1}^{3-p} & (p=0,1 ; q=0) \\
M\left(\bar{g}_{j+1}^{2}\left|\log \bar{g}_{j+1}\right|\right)^{-p}\left(\chi_{j+1} \bar{g}_{j+1}^{3}\right)^{1-q} & \left(\begin{array}{c}
p=0, q=1 \\
\text { or } p+q=2,3
\end{array}\right),\end{cases} \\
\left\|D_{V}^{p} D_{K}^{q} \psi_{j}\left(x_{j}\right)\right\|_{L^{p, q}} \leq \begin{cases}R \chi_{j+1} \bar{g}_{j+1}^{3} & \left(p=q=0 ; K_{j}=0\right) \\
M \chi_{j+1} \bar{g}_{j+1}^{2} & (p=1 ; q=0) \\
\kappa & (p=0, q=1) \\
M\left(\bar{g}_{j+1}^{2}\left|\log \bar{g}_{j+1}\right|\right)^{-p}\left(\chi_{j+1} \bar{g}_{j+1}^{3}\right)^{1-q} & (p+q=2,3) .\end{cases}
\end{gathered}
$$

The following theorem, which is illustrated by Figure 4, is a restatement of [7, Theorem 1.4 (with [7, Remark 1.5] for part (iii)), and its corollary is a restatement of [7, Corollary 1.8]. We make the simplifying assumption that $K_{0}=0$, which corresponds to our application with $K_{0}(X)=0$ for all non-empty polymers $X$. In the theorem, the sequence $\left(\bar{K}_{j}\right)_{j \in \mathbb{N}_{0}}$ is defined inductively by $\bar{K}_{j+1}=\psi_{j}\left(\bar{K}_{j}, \bar{V}_{j}\right)$, with $\bar{K}_{0}=0$. The existence of this sequence is established in [7, Lemma 1.3], which also gives

$$
\left\|\bar{K}_{j}\right\|_{\mathcal{W}_{j}} \leq a_{*} \chi_{j} \bar{g}_{j}^{3}
$$

for any $a_{*} \in(R /(1-\kappa \Omega), a]$, if $\bar{g}_{0}$ is chosen sufficiently small (depending on $a_{*}$ ).

Theorem 7.2. Fix a sequence of Banach spaces $\mathcal{W}_{j}$. Suppose that (A1-A3) hold with parameters given by $(a, h, \kappa, \Omega, M, R)$ and with $\bar{g}_{0}=\tilde{g}_{0}$. Let $a_{*} \in(R /(1-\kappa \Omega), a), b \in(0,1)$. There exists $h_{*}>0$ such that for any $h \geq h_{*}$, there exists $g_{*}>0$ such that if $\tilde{g}_{0} \in\left(0, g_{*}\right]$, there exists a neighbourhood $\mathrm{N}=\mathrm{N}\left(\tilde{g}_{0}\right) \subset \mathbb{R}$ of $\tilde{g}_{0}$ such that the following conclusions hold. 
(i) For initial condition $g_{0} \in \mathrm{N}$, there exists a global flow $\check{x}$ of $\Phi=(\bar{\varphi}+\rho, \psi)$ with $\left(\check{z}_{\infty}, \check{\mu}_{\infty}\right)=(0,0)$ such that, with $\bar{x}$ the unique flow of $\bar{\Phi}=(\bar{\varphi}, \psi)$ determined by the same boundary conditions,

$$
\begin{aligned}
\left|\check{g}_{j}-\bar{g}_{j}\right| & \leq b h \bar{g}_{j}^{2}\left|\log \bar{g}_{j}\right|, \\
\left|\check{z}_{j}-\bar{z}_{j}\right| & \leq b h \chi_{j} \bar{g}_{j}^{2}\left|\log \bar{g}_{j}\right|, \\
\left|\check{\mu}_{j}-\bar{\mu}_{j}\right| & \leq b h \chi_{j} \bar{g}_{j}^{2}\left|\log \bar{g}_{j}\right|, \\
\left\|K_{j}-\bar{K}_{j}\right\|_{\mathcal{W}_{j}} & \leq b\left(a-a_{*}\right) \chi_{j} \bar{g}_{j}^{3} .
\end{aligned}
$$

The sequence $\check{x}$ is the unique solution to (7.4) which obeys these boundary conditions and the bounds (7.11) -(7.14).

(ii) For every $j \in \mathbb{N}_{0}$, the $\operatorname{map}\left(\check{V}_{j}, K_{j}\right): \mathbf{N} \rightarrow \mathcal{V} \oplus \mathcal{W}_{j}$ is $C^{1}$ and obeys

$$
\frac{\partial \check{z}_{0}}{\partial g_{0}}=O(1), \quad \frac{\partial \check{\mu}_{0}}{\partial g_{0}}=O(1)
$$

(iii) The neighbourhood $\mathrm{N}\left(\tilde{g}_{0}\right)$ can be taken to be an interval centred at $\tilde{g}_{0}$, whose length $\varepsilon\left(\tilde{g}_{0}\right)>0$ depends only on $\tilde{g}_{0}$, the constants in (A1-A3), and $a_{*}, b, h$, and with $\varepsilon\left(\tilde{g}_{0}\right)$ bounded below away from 0 uniformly on compact subsets of $\tilde{g}_{0}>0$.

In particular, (7.10) and (7.14) imply that $\left\|K_{j}\right\|_{\mathcal{W}_{j}} \leq a \chi_{j} \bar{g}_{j}^{3}$ for all $j \in \mathbb{N}_{0}$. Theorem 7.2 concerns a single dynamical system $\Phi=\left(\Phi_{j}\right)_{j \in \mathbb{N}_{0}}$. Let $M_{\text {ext }}$ be a metric space of external parameters and assume now that the $\Phi_{j}$ depend continuously on an external parameter $m^{2} \in M_{\text {ext }}$, in the sense that the $\Phi_{j}$ are continuous maps $X_{j} \times M_{\text {ext }} \rightarrow X_{j+1}$. We say that $\Phi$ satisfies (A1-A3) uniformly if $\Phi_{j}\left(\cdot, m^{2}\right)$ satisfies $(\mathrm{A} 1-\mathrm{A} 3)$ for parameters $(a, h, \kappa, \Omega, R, M)$ independent of $m^{2} \in M_{\text {ext }}$. For the proof of Theorem 4.1, we apply the following extension to Theorem 7.2 , with $M_{\text {ext }} \subset[0, \delta)$.

Corollary 7.3. Assume that the $\Phi_{j}$ depend continuously on an external parameter $m^{2} \in M_{\text {ext }}$ and that Assumptions (A1-A3) hold uniformly in $m^{2}$. Let $x=x\left(m^{2}, g_{0}\right)=\left(V\left(m^{2}, g_{0}\right), K\left(m^{2}, g_{0}\right)\right)$ be the global flow for external parameter $m^{2}$ and initial condition $g_{0} \in \mathrm{N}\left(\tilde{g}_{0}\right)$ guaranteed by Theorem 7.2. Then $x_{j}$ is continuous in $\left(m^{2}, g_{0}\right)$ for each $j \in \mathbb{N}_{0}$, i.e., $\left(m^{2}, g_{0}\right) \mapsto V_{j}\left(m^{2}, g_{0}\right)$ is a continuous function into $\mathbb{R}^{3}$, and $\left(m^{2}, g_{0}\right) \mapsto K_{j}\left(m^{2}, g_{0}\right)$ is a continuous function into $\mathcal{W}_{j}$.

\subsection{Proof of Proposition 7.1}

We prove Proposition 7.1 by applying Theorem 7.2 to the infinite volume evolution map (7.3) for the transformed variables, with the verification of (A3) via Theorem 6.4. The proof is divided into three main steps:

Step 1. Construction of the maps $z_{0}^{c}, \mu_{0}^{c}$ and proof of the estimates for $\left(V_{j}, K_{j}\right)$ for all $j \in \mathbb{N}$. Step 2. Proof of continuity of $z_{0}^{c}, \mu_{0}^{c}$ in the interior of their domains, i.e., $\left(m^{2}, g_{0}\right) \in(0, \delta)^{2}$.

Step 3. Proof that $z_{0}^{c}, \mu_{0}^{c}$ are continuous on $[0, \delta)^{2}$.

For the regularity statements of steps 2-3, a difficulty is that Theorem 6.4 involves the restrictions $\tilde{m}^{2} \in \mathbb{I}_{+}=[0, \delta)$ and $m^{2} \in \tilde{\mathbb{I}}_{+}\left(\tilde{m}^{2}\right)$, which maintain the mass $\tilde{m}^{2}$ appearing in the norm close to the mass $m^{2}$ appearing in (7.3) through its implicit dependence on the original covariance $\left(-\Delta+m^{2}\right)^{-1}$. To prove mass continuity of $z_{0}^{c}, \mu_{0}^{c}$ in $m^{2} \in[0, \delta)$, we must prove some compatibility in this respect. Similarly, Theorem 7.2 permits only local variation of $g_{0}$, whereas we must prove 


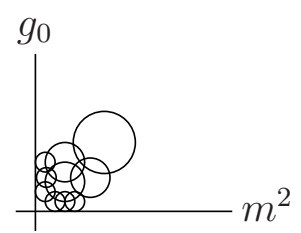

Figure 5: To construct a continuous solution on $\left(m^{2}, g_{0}\right) \in(0, \delta)^{2}$, we first construct solutions in small neighbourhoods of a fixed $\left(\tilde{m}^{2}, \tilde{g}_{0}\right)$, and show compatibility of these solutions.

continuity in $g_{0} \in[0, \delta)$. This is illustrated by Figure 5. To deal with these difficulties, although we aim to prove $\left(V_{j}, K_{j}\right) \in \mathbb{D}_{j}\left(s_{j}\right)$ with $s_{j}=\left(m^{2}, \tilde{g}_{j}\left(m^{2}, g_{0}\right)\right)$, we consider a wider class of parameters and spaces. To simplify the notation, we write

$$
\mathcal{W}_{j}^{0}\left(\tilde{m}^{2}, \tilde{g}_{0}\right)=\mathcal{W}_{j}\left(\tilde{m}^{2}, \tilde{g}_{j}\left(\tilde{m}^{2}, \tilde{g}_{0}\right), \mathbb{Z}^{d}\right)
$$

where here $\left(\tilde{m}^{2}, \tilde{g}_{0}\right)$ defines the sequence $\left(\tilde{g}_{j}\right)$ via $(\underline{6.15})$.

\subsubsection{Preliminaries: the sequence $\tilde{g}$ and norms}

The proof uses the properties established in the next lemma, for the sequence $\tilde{g}_{j}$ defined in (6.15). The proof of Lemma 7.4 uses elementary calculus only.

Lemma 7.4. (i) There exists $\delta>0$ such that uniformly in $\left(m^{2}, g_{0}\right) \in(0, \delta)^{2}$ and in $j \in \mathbb{N}_{0}$,

$$
\tilde{g}_{j}\left(m^{2}, g_{0}\right)=\bar{g}_{j}\left(m^{2}, g_{0}\right)+O\left(\bar{g}_{j}^{2}\left(m^{2}, g_{0}\right)\right) .
$$

(ii) For sufficiently small $\theta>0$, if $\left|m^{2}-\hat{m}^{2}\right| \leq \theta \hat{m}^{2}$ and $\left|g_{0}-\hat{g}_{0}\right| \leq \theta \hat{g}_{0}$, then

$$
\left|\tilde{g}_{j}\left(m^{2}, g_{0}\right)-\tilde{g}_{j}\left(\hat{m}^{2}, \hat{g}_{0}\right)\right| \leq\left(\theta+O\left(g_{0}\right)\right) \tilde{g}_{j}\left(\hat{m}^{2}, \hat{g}_{0}\right) \text {. }
$$

(iii) For $j \in \mathbb{N}_{0}, \tilde{g}_{j}\left(m^{2}, g_{0}\right)$ is monotone increasing in each of $m^{2}$ and $g_{0}$.

Proof. (i) We use dots to denote derivatives with respect to $m^{2}$. By [6. Lemma 6.3(b)], $\dot{\beta}_{l}=O\left(L^{2 l}\right)$ (with $L$-dependent constant). Differentiation of (6.2) gives $\dot{\bar{g}}_{j+1}=\dot{\bar{g}}_{j}\left(1-2 \beta_{j} \bar{g}_{j}\right)-\dot{\beta}_{j} \bar{g}_{j}^{2}$. We solve the recursion and then apply [7, Lemma 2.1(iii)] to obtain

$$
\dot{\bar{g}}_{j}=-\sum_{l=0}^{j-1} \prod_{k=l}^{j}\left(1-2 \beta_{j} \bar{g}_{j}\right) \dot{\beta}_{l} \bar{g}_{l}^{2}=-\left(1+O\left(g_{0}\right)\right) \sum_{l=0}^{j-1}\left(\frac{\bar{g}_{j}}{\bar{g}_{l}}\right)^{2} \dot{\beta}_{l} \bar{g}_{l}^{2}=O\left(\bar{g}_{j}^{2} L^{2 j}\right) .
$$

By continuity, $\bar{g}_{j}$ achieves its maximum on $\left[0, \hat{m}^{2}\right]$ at some $m_{*}^{2}$ (depending on $j$ ). For $j \leq j_{m}$, by integrating the derivative we obtain

$$
\left|\bar{g}_{j}(0)-\bar{g}_{j}\left(m^{2}\right)\right| \leq O\left(\bar{g}_{j}^{2}\left(m_{*}^{2}\right)\right) .
$$

Thus $\bar{g}_{j}\left(m_{*}^{2}\right) \leq \bar{g}_{j}(0)+O\left(\bar{g}_{j}\left(m_{*}^{2}\right)^{2}\right)$. Since $\bar{g}_{j}\left(m_{*}^{2}\right)=O\left(\bar{g}_{0}\right)$ (by [7, Lemma 2.1(i)]), it follows that $\bar{g}_{j}\left(m_{*}^{2}\right) \leq\left(1+O\left(\bar{g}_{0}\right)\right) \bar{g}_{j}(0)$, and hence $\bar{g}_{j}\left(m^{2}\right)=\bar{g}_{j}(0)+O\left(\bar{g}_{j}(0)^{2}\right)$ for $j \leq j_{m}$ as claimed. 
For $j \geq j_{m}$, we iterate (6.2), and use $\bar{g}_{l} \leq \bar{g}_{j_{m}}$ for $l \geq j_{m}$ (which is immediate from $\beta_{k} \geq 0$ ) and $\sum_{l=j_{m}}^{\infty}\left|\beta_{l}\right|=O(1)$ (by [6, Lemma 6.2]), to obtain

$$
\bar{g}_{j}=\bar{g}_{j_{m}} \prod_{l=j_{m}}^{j-1}\left(1-\beta_{l} \bar{g}_{l}\right)=\bar{g}_{j_{m}} \exp \left(\sum_{l=j_{m}}^{j-1} O\left(\beta_{l} \bar{g}_{l}\right)\right)=\bar{g}_{j_{m}} \exp \left(O\left(\bar{g}_{j_{m}}\right)\right)=\bar{g}_{j_{m}}+O\left(\bar{g}_{j_{m}}^{2}\right),
$$

and the proof of (i) is complete.

(ii) Using $\log (1+t) \leq t$ for $t \geq 0$, we first observe that for sufficiently small $\theta>0$,

$$
\left|j_{\hat{m}}-j_{m}\right| \leq 1+\left|\log _{L^{2}} \hat{m}^{2}-\log _{L^{2}} m^{2}\right| \leq 1+\frac{\left|\hat{m}^{2}-m^{2}\right|}{m^{2} \log L^{2}} \leq 1+O(\theta)<2 .
$$

Thus, since $j_{m}$ is an integer, $\left|j_{\hat{m}}-j_{m}\right| \leq 1$. Let $j_{m}^{*}(j)=j_{m}$ if $j \geq j_{m}$ and $j_{m}^{*}(j)=j$ otherwise. Then also $\left|j_{m}^{*}-j_{\hat{m}}^{*}\right| \leq\left|j_{m}-j_{\hat{m}}\right| \leq 1$. By (6.15) and the triangle inequality,

$$
\begin{aligned}
\tilde{g}_{j}\left(g_{0}, m^{2}\right)-\tilde{g}_{j}\left(\hat{g}_{0}, \hat{m}^{2}\right) & =\bar{g}_{j_{m}^{*}}\left(g_{0}\right)-\bar{g}_{j_{\hat{m}}^{*}}\left(\hat{g}_{0}\right) \\
& \leq\left|\bar{g}_{j_{m}^{*}}\left(g_{0}\right)-\bar{g}_{j_{\hat{m}}^{*}}\left(g_{0}\right)\right|+\left|\bar{g}_{j_{\hat{m}}^{*}}\left(g_{0}\right)-\bar{g}_{j_{\hat{m}}^{*}}\left(\hat{g}_{0}\right)\right| .
\end{aligned}
$$

By (6.2), $\left|\bar{g}_{j_{m}^{*}}-\bar{g}_{j_{\hat{m}}^{*}}\right| \leq O\left(\bar{g}_{j_{\tilde{m}}^{*}}^{2}\right)$. Also, it is shown in [7, Lemma 2.1(iv)] that if $\left|g_{0}-\hat{g}_{0}\right| \leq \theta \hat{g}_{0}$ then

$$
\left|\bar{g}_{j}\left(g_{0}\right)-\bar{g}_{j}\left(\hat{g}_{0}\right)\right| \leq \theta\left(1+O\left(g_{0}\right)\right) \bar{g}_{j}\left(g_{0}\right)
$$

The combination of the above estimates gives

$$
\left|\tilde{g}_{j}\left(g_{0}, m^{2}\right)-\tilde{g}_{j}\left(\hat{g}_{0}, \hat{m}^{2}\right)\right| \leq\left(\theta+O\left(g_{0}\right)\right) \tilde{g}_{j}\left(\hat{g}_{0}, \hat{m}^{2}\right)
$$

which is (7.18).

(iii) The sequence $\bar{g}$ is monotone increasing in $g_{0}$ (by [7, (2.50)]), and thus the sequence $\tilde{g}$ is also monotone increasing in $g_{0}$. Also, the sequence $\bar{g}$ is decreasing in $j$ since $\beta_{k} \geq 0$ for all $k$, while $j_{m}$ is decreasing in $m^{2}$, so $\tilde{g}$ is increasing in $m^{2}$.

In preparation for step 2, we make the following observation. It is proved in [24, Lemma 1.9 ] $\left(\right.$ with $1+O\left(\tilde{g}-\tilde{g}^{\prime}\right) \leq 2$ ) that there exists $\delta>0$ such that, whenever $\tilde{m}^{2} \leq \tilde{m}^{\prime 2}$ and $\tilde{g}^{\prime} \leq \tilde{g} \leq(1+\delta) \tilde{g}^{\prime}$,

$$
\|\cdot\|_{\mathcal{W}_{j}\left(\tilde{m}^{2}, \tilde{g}\right)} \leq 2\|\cdot\|_{\mathcal{W}_{j}\left(\tilde{m}^{\prime 2}, \tilde{g}^{\prime}\right)}
$$

Therefore, by Lemma 7.4(ii-iii), there exists $\delta>0$ such that if $\tilde{m}^{2} \leq m^{2}$ and $g_{0} \leq \tilde{g}_{0} \leq(1+\delta) g_{0}$, then, for all $g_{0} \in(0, \delta)$,

$$
\|\cdot\|_{\mathcal{W}_{j}^{0}\left(\tilde{m}^{2}, \tilde{g}_{0}\right)} \leq 2\|\cdot\|_{\mathcal{W}_{j}^{0}\left(m^{2}, g_{0}\right)}
$$

with $\mathcal{W}^{0}$ defined in (7.16).

In preparation for step 3, we recall from [24, Lemma 1.9] that $\|\cdot\|_{\mathcal{W}_{j}(\tilde{m}, \tilde{g})}=\|\cdot\|_{\mathcal{W}_{j}(0, \tilde{g})}$ if $j \leq j_{\Omega}$. In addition, if $j \leq j_{m}$ then $\tilde{g}_{j}\left(m^{2}, g_{0}\right)=\tilde{g}_{j}\left(0, g_{0}\right)$ by definition in (6.15). Therefore the scale- $j$ norm is independent of $m^{2}$ as long as $j \leq \min \left\{j_{\Omega}, j_{m}\right\}$. As discussed below (6.6), the mass scale $j_{m}$ differs from $j_{\Omega}$ by at most a constant, so we conclude that there is a constant $c$ such that

$$
\|\cdot\|_{\mathcal{W}_{j}^{0}\left(m^{2}, g_{0}\right)}=\|\cdot\|_{\mathcal{W}_{j}^{0}\left(0, g_{0}\right)} \quad \text { for } m^{2} \leq c L^{-2 j}
$$




\subsubsection{Step 1: construction of the maps $z_{0}^{c}, \mu_{0}^{c}$}

Step 1. We construct the maps $z_{0}^{c}, \mu_{0}^{c}$ and show that for initial conditions (7.2), (7.1) is valid and $\left(V_{j}, K_{j}\right) \in \mathbb{D}_{j}\left(s_{j}\right)$ for all $j \in \mathbb{N}_{0}$. As explained in Section 6.6, this is equivalent to showing that there exist $\left(\check{V}_{j}, K_{j}\right) \in \check{\mathbb{D}}_{j}\left(s_{j}\right)$ satisfying (17.4). We also show the estimates stated for $V_{j}, \check{V}_{j}$.

We fix $\left(\tilde{m}^{2}, \tilde{g}_{0}\right) \in[0, \delta) \times(0, \delta)$. To apply Theorem 7.2 to the maps (17.3) with the $\mathcal{W}_{j}$ spaces given by $\mathcal{W}_{j}^{0}\left(\tilde{m}^{2}, \tilde{g}_{0}\right)$, we must verify Assumptions (A1-A3) with this choice. Assumptions (A1A2) for the maps $\bar{\varphi}$ have already been seen to be satisfied in Section 6.1. We apply Theorem 6.4 to verify (A3) with $\mathcal{W}_{j}=\mathcal{W}_{j}^{0}\left(\tilde{m}^{2}, \tilde{g}_{0}\right)$. Application of Theorem 7.2 with a single value $\left(\tilde{m}^{2}, \tilde{g}_{0}\right)$ then produces a local solution, i.e., sequences $\left(\check{V}_{j}, K_{j}\right) \in \check{\mathbb{D}}_{j}$ defined for each $\left(m^{2}, g_{0}\right)$ in a neighbourhood of $\left(\tilde{m}^{2}, \tilde{g}_{0}\right)$. In steps $2-3$, we subsequently show that these local solutions can be combined into a single continuous solution of $\left(m^{2}, g_{0}\right) \in[0, \delta)^{2}$. The details are as follows.

Recall the domain $\mathbb{D}_{j}$ of (6.17). We write $\mathbb{D}_{j}^{0}\left(\tilde{m}^{2}, \tilde{g}_{0}\right)$ instead of $\mathbb{D}_{j}\left(\tilde{m}^{2}, \tilde{g}_{j}\left(\tilde{m}^{2}, \tilde{g}_{0}\right)\right)$. Recall also the domain $D_{j}$ of (7.7). Explicitly, with $\tilde{\chi}_{j}=\chi_{j}\left(\tilde{m}^{2}\right)$,

$$
\begin{aligned}
\mathbb{D}_{j}^{0}\left(\tilde{m}^{2}, \tilde{g}_{0}\right)=\{ & \left.\left(g_{j}, z_{j}, \mu_{j}\right): C_{\mathcal{D}}^{-1} \tilde{g}_{j}<g_{j}<C_{\mathcal{D}} \tilde{g}_{j},\left|z_{j}\right|,\left|\mu_{j}\right|<C_{\mathcal{D}} \tilde{g}_{j}\right\} \\
& \times B_{\mathcal{W}_{j}^{0}\left(\tilde{m}^{2}, \tilde{g}_{0}\right)}\left(\alpha \tilde{\chi}_{j} \tilde{g}_{j}^{3}\right), \\
D_{j}\left(\tilde{m}^{2}, \tilde{g}_{0}\right)=\{ & \left(\check{g}_{j}, \check{z}_{j}, \check{\mu}_{j}\right):\left|\check{g}_{j}-\bar{g}_{j}\right| \leq h \bar{g}_{j}^{2}\left|\log \bar{g}_{j}\right|, \\
& \left.\left|\check{z}_{j}-\bar{z}_{j}\right|,\left|\check{\mu}_{j}-\bar{\mu}_{j}\right| \leq h \tilde{\chi}_{j} \bar{g}_{j}^{2}\left|\log \bar{g}_{j}\right|\right\} \\
& \times B_{\mathcal{W}_{j}^{0}\left(\tilde{m}^{2}, \tilde{g}_{0}\right)}\left(a \tilde{\chi}_{j} \bar{g}_{j}^{3}\right) .
\end{aligned}
$$

Set $a=\alpha$. By Lemma 7.4(i), for any fixed $C_{\mathcal{D}}>1$ and $h>0$ (with $\delta$ chosen small depending on $h$ ), the domains of (17.29) -(7.30) obey $D_{j} \subset T_{j}\left(\mathbb{D}_{j}^{0}\right)$. Therefore, for any $(\check{V}, K) \in D_{j}$, by Corollary 6.5 , $\rho=\check{R}_{+}, \psi=\check{K}_{+}$obey the bounds of Theorem 6.4 (namely those of Theorem 6.3). For convenience, we recall the bounds for $\check{K}_{+}$from (6.24), which are more delicate than those for $\check{R}_{+}$:

$$
\left\|D_{V}^{p} D_{K}^{q} \check{K}_{+}\right\|_{L^{p, q}} \leq \begin{cases}M \tilde{\chi}_{j+1} \tilde{g}_{j+1}^{3-p} & (p \geq 0) \\ \kappa & (p=0, q=1) \\ M \tilde{g}_{j+1}^{-p}\left(\tilde{\chi}_{j+1} \tilde{g}_{j+1}^{10 / 4}\right)^{1-q} & (p \geq 0, q \geq 1)\end{cases}
$$

As stated above the statement of Proposition 17.1, we have made the choice $\alpha=4 M$. Also, since $\kappa=O\left(L^{-1}\right)$, we can and do assume that $\kappa<\frac{1}{2} \Omega^{-1}$. Then (7.31) implies the bounds of Assumption (A3) for $\check{K}$, namely

$$
\left\|D_{V}^{p} D_{K}^{q} \check{K}_{+}\right\|_{L^{p, q}} \leq \begin{cases}R^{\prime} \chi_{j+1} \bar{g}_{j+1}^{3} & \left(p=q=0 ; K_{j}=0\right) \\ M^{\prime} \chi_{j+1} \bar{g}_{j+1}^{2} & (p=1 ; q=0) \\ \kappa & (p=0, q=1) \\ M^{\prime}\left(\bar{g}_{j+1}^{2}\left|\log \bar{g}_{j+1}\right|\right)^{-p}\left(\chi_{j+1} \bar{g}_{j+1}^{3}\right)^{1-q} & (p+q=2,3)\end{cases}
$$

with constants $M^{\prime}=2 M$ (to absorb a factor 2 in replacing $\tilde{g}_{j}$ by $\bar{g}_{j}=\bar{g}_{j}\left(1+O\left(\bar{g}_{j}\right)\right)$, and $R^{\prime}=2 M=$ $\frac{1}{2} a<a\left(1-\kappa \Omega\right.$ ) (the factor 2 is again to replace $\tilde{g}_{j}$ by $\bar{g}_{j}$, the second equality is our choice $a=4 M$, and the inequality follows since $\kappa \Omega<\frac{1}{2}$ ). Note that the powers in (7.32) are less restrictive than in (7.31). The conclusion of the previous discussion is that, for any $\left(\tilde{m}^{2}, \tilde{g}_{0}\right) \in[0, \delta) \times(0, \delta)$, the maps 
$\Phi\left(\cdot, m^{2}\right)$ with $m^{2} \in \tilde{\mathbb{I}}_{+}\left(\tilde{m}^{2}\right)$ satisfy $(\mathrm{A} 3)$ with norms $\mathcal{W}_{j}^{0}\left(\tilde{m}^{2}, \tilde{g}_{0}\right)$ and parameters $\left(a, h, \kappa, \Omega, R^{\prime}, M^{\prime}\right)$ depending on the constants in Theorem 6.4, but not on any of $\tilde{m}^{2}, m^{2}, \tilde{g}_{0}, g_{0}$.

We choose $b=\frac{1}{4}$ (somewhat arbitrarily), $h>h_{*}$ large enough, $\delta \leq g_{*}$, and apply Theorem 7.2 to conclude that for each $\left(\tilde{m}^{2}, \tilde{g}_{0}\right) \in(0, \delta)^{2}$, there is a neighbourhood $N\left(\tilde{m}^{2}, \tilde{g}_{0}\right)=\tilde{\mathbb{I}}_{+}\left(\tilde{m}^{2}\right) \times \mathrm{N}\left(\tilde{g}_{0}\right) \subset$ $(0, \delta)^{2}$ of $\left(\tilde{m}^{2}, \tilde{g}_{0}\right)$ such that, given $\left(m^{2}, g_{0}\right) \in N\left(\tilde{m}^{2}, \tilde{g}_{0}\right)$, there are solutions to (7.4) with maps $\Phi_{j}=\Phi_{j}\left(\cdot, m^{2}\right)$ that satisfy the boundary conditions (17.5) specified by the parameter $g_{0}$. We denote these solutions by $\check{x}^{d}\left(\tilde{m}^{2}, \tilde{g}_{0} ; m^{2}, g_{0}\right)$ where the argument $\left(\tilde{m}^{2}, \tilde{g}_{0}\right)$ indicates the dependence on the Banach spaces $\mathcal{W}_{j}^{0}\left(\tilde{m}^{2}, \tilde{g}_{0}\right)$ to which we apply Theorem 7.2 , and where $\left(m^{2}, g_{0}\right)$ refers to the mass parameter $m^{2}$ of $\Phi_{j}$ and the initial condition $g_{0}$.

Choosing $\left(\tilde{m}^{2}, \tilde{g}\right)=\left(m^{2}, g\right)$, we define a map $\check{x}_{j}^{c}:[0, \delta) \times(0, \delta) \rightarrow \mathcal{V} \oplus \mathcal{K}_{j}\left(\mathbb{Z}^{d}\right)$ by

$$
\check{x}_{j}^{c}\left(m^{2}, g_{0}\right)=\check{x}_{j}^{d}\left(m^{2}, g_{0} ; m^{2}, g_{0}\right) \in \mathcal{V} \oplus \mathcal{W}_{j}^{0}\left(m^{2}, g_{0}\right) \subset \mathcal{V} \oplus \mathcal{K}_{j}\left(\mathbb{Z}^{d}\right)
$$

We extend this map to $[0, \delta)^{2}$ by setting $\check{x}_{j}^{c}\left(m^{2}, 0\right)=(0,0)$. In particular, restriction to the $\left(z_{0}, \mu_{0}\right)$-component produces two real-valued maps $z_{0}^{c}:[0, \delta)^{2} \rightarrow \mathbb{R}$ and $\mu_{0}^{c}:[0, \delta)^{2} \rightarrow \mathbb{R}$ such that $z_{0}^{c}\left(m^{2}, 0\right)=\mu_{0}^{c}\left(m^{2}, g_{0}\right)=0$, and the flow with initial condition (17.2) satisfies $\left(V_{j}, K_{j}\right) \in \mathbb{D}_{j}\left(s_{j}\right)$ for all $j \in \mathbb{N}_{0}$, as desired.

In particular, this implies $\check{g}_{j}=O\left(\bar{g}_{j}\right)$. Since $\bar{z}_{j}, \bar{\mu}_{j}=O\left(\chi_{j} \bar{g}_{j}\right)$, by Proposition 6.1, (7.12)-(7.13) also show $\check{z}_{j}, \check{\mu}_{j}=O\left(\chi_{j} \bar{g}_{j}\right)$. By ([6.1), it then follows that $g_{j}=O\left(\bar{g}_{j}\right)$. Furthermore, by [ $\underline{6}$, (6.92)(6.93)], $\check{\mu}_{j}=\mu_{j}+O\left(\mu_{j}\right)^{2}$ and $\check{z}_{j}=z_{j}+O\left(z_{j} \mu_{j}\right)+O\left(\mu_{j}^{2}\right)$, and $z_{j}, \mu_{j}=O\left(\chi_{j} \bar{g}_{j}\right)$ follows.

\subsubsection{Step 2: regularity in the interior}

In preparation for step 2 of the proof, we make the following two observations:

(a) The neighbourhood $N\left(\tilde{m}^{2}, \tilde{g}_{0}\right)=\tilde{\mathbb{I}}_{+}\left(\tilde{m}^{2}\right) \times N\left(\tilde{g}_{0}\right)$ in the proof of (i-ii) can be taken instead to be of the form $N\left(\tilde{m}^{2}, \tilde{g}_{0}\right)=\left(\tilde{m}^{2}-\varepsilon\left(\tilde{m}^{2}, \tilde{g}\right), \tilde{m}^{2}+\varepsilon\left(\tilde{m}^{2}, \tilde{g}\right)\right) \times\left(\tilde{g}-\varepsilon\left(\tilde{m}^{2}, \tilde{g}\right), \tilde{g}+\varepsilon\left(\tilde{m}^{2}, \tilde{g}\right)\right)$, with $\varepsilon\left(\tilde{m}^{2}, \tilde{g}\right)>0$ bounded away from 0 uniformly on compact subsets of $(0, \delta)^{2}$.

(b) The flexibility to choose any $b \in(0,1)$ in Theorem 7.2 can be used to enhance the statement of Theorem 7.2, as follows. We apply Theorem 7.2 with two choices of $b$, namely $b_{1}=\frac{1}{2}$ and $b_{2}=\frac{1}{4}$. The values of $h_{*}, g_{*}$ in Theorem 7.2 depend on $b$, and we assume that $h>\max \left\{h_{*}\left(b_{1}\right), h_{*}\left(b_{2}\right)\right\}$ but otherwise the choice of $h$ is arbitrary, and $\delta \leq \min \left\{g_{*}\left(b_{1}\right), g_{*}\left(b_{2}\right)\right\}$. As in the proof of (i-ii), for $\left(\tilde{m}^{2}, \tilde{g}_{0}\right) \in(0, \delta)^{2}$, there exists a neighbourhood $N\left(\tilde{m}^{2}, \tilde{g}_{0}\right)$ such that for each $\left(m^{2}, g_{0}\right) \in N\left(\tilde{m}^{2}, \tilde{g}_{0}\right)$, there exists a solution that satisfies (17.11)-(17.14) with $b=b_{2}=\frac{1}{4}$ and $\mathcal{W}_{j}=\mathcal{W}_{j}^{0}\left(\tilde{m}^{2}, \tilde{g}_{0}\right)$, by applying the existence statement of Theorem 7.2 with the latter parameters. Moreover, any solution that satisfies (7.11)-(7.14) with $b=b_{1}=\frac{1}{2}$ and $\mathcal{W}_{j}=\mathcal{W}_{j}^{0}\left(\tilde{m}^{2}, \tilde{g}_{0}\right)$ is unique, by applying the uniqueness statement of Theorem 7.2 with $b=b_{1}=\frac{1}{2}$, and hence must, in fact, satisfy (7.14) with the smaller value of the parameter $b=b_{2}=\frac{1}{4}$ and therefore be identical to the solution produced when $b=b_{2}$.

Step 2. We show that $z_{0}^{c}, \mu_{0}^{c}$ are continuous in $\left(m^{2}, g_{0}\right) \in(0, \delta)^{2}$, differentiable in $g_{0} \in(0, \delta)$, with uniformly bounded $g_{0}$-derivative.

We set $b=b_{2}=\frac{1}{4}$ as in step 1 . We fix some $\left(\hat{m}^{2}, \hat{g}_{0}\right) \in(0, \delta)^{2}$ and show that $z_{0}^{c}, \mu_{0}^{c}$ are continuous in both variables and differentiable in $g_{0}$ at this $\left(\hat{m}^{2}, \hat{g}_{0}\right)$. By Corollary 7.3 , for any $\left(\tilde{m}^{2}, \tilde{g}_{0}\right) \in(0, \delta)^{2}, \check{x}_{j}^{d}\left(\tilde{m}^{2}, \tilde{g}_{0} ; m^{2}, g_{0}\right)$ is continuous in $\left(m^{2}, g_{0}\right) \in N\left(\tilde{m}^{2}, \tilde{g}_{0}\right)$, as a map taking values 
in the Banach space $\mathcal{V} \oplus \mathcal{W}_{j}^{0}\left(\tilde{m}^{2}, \tilde{g}_{0}\right)$. Moreover, by Theorem 7.2 (ii), this map is differentiable in $g_{0}$, for fixed $\left(\tilde{m}^{2}, \tilde{g}_{0}, m^{2}\right)$, and its derivative is bounded uniformly in $\left(\tilde{m}^{2}, m^{2}, \tilde{g}_{0}, g_{0}\right)$. It therefore suffices to show that, given $\left(\hat{m}^{2}, \hat{g}_{0}\right)$, there exists $\left(\tilde{m}^{2}, \tilde{g}_{0}\right)$ such that $\left(\hat{m}^{2}, \hat{g}_{0}\right) \in N\left(\tilde{m}^{2}, \tilde{g}_{0}\right)$, and that for all $\left(m^{2}, g_{0}\right)$ in a neighbourhood of $\left(\hat{m}^{2}, \hat{g}_{0}\right), \check{x}_{j}^{d}\left(m^{2}, g_{0} ; m^{2}, g_{0}\right)=\check{x}_{j}^{d}\left(\tilde{m}^{2}, \tilde{g}_{0} ; m^{2}, g_{0}\right)$. Such a neighbourhood is constructed in the next paragraph. Assuming its existence, the continuity in $\left(m^{2}, g_{0}\right)$ and the differentiability in $g_{0}$ of $\check{x}_{j}^{c}\left(m^{2}, g_{0}\right)$ follow from the already established properties of $\check{x}_{j}^{d}\left(\tilde{m}^{2}, \tilde{g}_{0} ; \cdot, \cdot\right)$.

We now choose $\left(\tilde{m}, \tilde{g}_{0}\right)$ and the neighbourhood of $\left(\hat{m}^{2}, \hat{g}_{0}\right)$, discussed in the previous paragraph. By item (a) above, given $\left(\hat{m}^{2}, \hat{g}_{0}\right)$, the radius of $N\left(\tilde{m}^{2}, \tilde{g}_{0}\right)$ is uniformly bounded below by some $\hat{\varepsilon}>0$ on the compact set $\tilde{m}^{2} \in\left[\frac{1}{2} \hat{m}^{2}, \hat{m}^{2}\right], \tilde{g}_{0} \in\left[\hat{g}_{0},(1+\delta) \hat{g}_{0}\right]$. We assume that $\hat{\varepsilon}<\max \left\{\delta, \frac{1}{2}\right\}$ and choose $\tilde{m}^{2}=\hat{m}^{2}-\frac{1}{2} \hat{\varepsilon}$ and $\tilde{g}_{0}=\hat{g}_{0}+\frac{1}{2} \hat{\varepsilon}$. Then $N\left(\tilde{m}^{2}, \tilde{g}_{0}\right) \supset\left[\hat{m}^{2}-\frac{1}{4} \hat{\varepsilon}, \hat{m}^{2}+\frac{1}{4} \hat{\varepsilon}\right] \times\left[\hat{g}_{0}-\frac{1}{4} \hat{\varepsilon}, \hat{g}_{0}+\frac{1}{4} \hat{\varepsilon}\right]=Q$. By definition, $\tilde{m}^{2} \leq m^{2}$ and $g_{0} \leq \tilde{g}_{0} \leq(1+\delta) g_{0}$ hold for all $\left(m^{2}, g_{0}\right) \in Q$. Thus, for $\left(m^{2}, g_{0}\right) \in Q$, by (7.27),

$$
\begin{aligned}
& \left\|K^{c}\left(m^{2}, g_{0} ; m^{2}, g_{0}\right)-\bar{K}\left(m^{2}, g_{0}\right)\right\|_{\mathcal{W}_{j}^{0}\left(\tilde{m}^{2}, \tilde{g}_{0}\right)} \\
& \quad \leq 2\left\|K^{c}\left(m^{2}, g_{0} ; m^{2}, g_{0}\right)-\bar{K}\left(m^{2}, g_{0}\right)\right\|_{\mathcal{W}_{j}^{0}\left(m^{2}, g_{0}\right)} \\
& \quad \leq 2 b_{2}\left(a-a_{*}\right) \bar{g}_{j}^{3}=b_{1}\left(a-a_{*}\right) \bar{g}_{j}^{3},
\end{aligned}
$$

and the bounds (7.11) (7.13) hold with $b=b_{2}<b_{1}$. As discussed in item (b) above, solutions for which (7.34) and (7.11)-(17.13) hold with $b_{1}$ are unique, from which we conclude that $\check{x}^{d}\left(\tilde{m}^{2}, \tilde{g}_{0} ; m^{2}, g_{0}\right)=\check{x}^{d}\left(m^{2}, g_{0} ; m^{2}, g_{0}\right)$ as desired.

\subsubsection{Step 3: regularity at the boundary}

Step 3. We show that $z_{0}^{c}, \mu_{0}^{c}$ are continuous as $g_{0} \downarrow 0$ or as $m^{2} \downarrow 0$.

The bounds $\mu_{0}^{c}, z_{0}^{c}=O\left(g_{0}\right)$, which hold uniformly in $m^{2}$, imply that $\mu_{0}^{c}, z_{0}^{c} \rightarrow 0$ as $\left(m^{2}, g_{0}\right) \rightarrow$ $\left(\hat{m}^{2}, 0\right)$ for some $\hat{m}^{2} \in[0, \delta)$. The continuity in the limit $g_{0} \downarrow 0$ is immediate from this.

For continuity of $\mu_{0}^{c}, z_{0}^{c}$ as $\left(m^{2}, g_{0}\right) \rightarrow\left(0, \hat{g}_{0}\right)$ for some $\hat{g}_{0} \in(0, \delta)$, we show that $\check{x}_{0}\left(m^{2}, g_{0}\right) \rightarrow$ $\check{x}_{0}\left(0, \hat{g}_{0}\right)$ by adapting the argument used in the proof of Corollary 7.3 (given in [7, Corollary 1.8]), as follows. First, we recall that

$$
\begin{aligned}
\left|\check{g}_{j}\left(m^{2}, g_{0}\right)-\bar{g}_{j}\left(m^{2}, g_{0}\right)\right| & \leq b h \bar{g}_{j}\left(m^{2}\right)^{2}\left|\log \bar{g}_{j}\left(m^{2}, g_{0}\right)\right| \\
\left|\check{\mu}_{j}\left(m^{2}, g_{0}\right)-\bar{\mu}_{j}\left(m^{2}, g_{0}\right)\right| & \leq b h \chi_{j}\left(m^{2}\right) \bar{g}_{j}\left(m^{2}, g_{0}\right)^{2}\left|\log \bar{g}_{j}\left(m^{2}, g_{0}\right)\right|, \\
\left|\check{z}_{j}\left(m^{2}, g_{0}\right)-\bar{z}_{j}\left(m^{2}, g_{0}\right)\right| & \leq b h \chi_{j}\left(m^{2}\right) \bar{g}_{j}\left(m^{2}, g_{0}\right)^{2}\left|\log \bar{g}_{j}\left(m^{2}, g_{0}\right)\right|, \\
\left\|K_{j}\left(m^{2}, g_{0}\right)-\bar{K}_{j}\left(m^{2}, g_{0}\right)\right\|_{\mathcal{W}_{j}^{0}\left(m^{2}, g_{0}\right)} & \leq b\left(a-a_{*}\right) \chi_{j}\left(m^{2}\right) \bar{g}_{j}\left(m^{2}, g_{0}\right)^{3} .
\end{aligned}
$$

By Proposition 6.1, $\bar{V}_{j}\left(m^{2}, g_{0}\right)$ is continuous in $\left(m^{2}, g_{0}^{2}\right) \in[0, \delta]$, and thus in particular $\bar{V}_{0}\left(m^{2}, g_{0}^{2}\right)$ is uniformly bounded for $\left(m^{2}, g_{0}^{2}\right) \in[0, \delta]^{2}$. With (7.35)-(7.37), we see that $\breve{V}_{0}\left(m^{2}, g_{0}\right)$ is therefore also uniformly bounded. Thus, for every sequence $\left(m^{2}, g_{0}\right) \rightarrow\left(0, \hat{g}_{0}\right), \check{V}_{0}\left(m^{2}, g_{0}\right)$ has a limit point. It suffices to show that any such limit point is equal to $\check{V}_{0}\left(0, \hat{g}_{0}\right)$. To show this uniqueness, we fix an arbitrary limit point $V_{0}^{*}$ and a sequence $\left(m^{2}, g_{0}\right) \rightarrow\left(0, \hat{g}_{0}\right)$ such that $\check{V}_{0}\left(m^{2}, g_{0}\right) \rightarrow V_{0}^{*}$. We also set $K_{0}^{*}=K_{0}=0$.

Then we define $x_{j}^{*}=\left(V_{j}^{*}, K_{j}^{*}\right)$ by inductive application of $\Phi_{j}(\cdot, 0)$ starting from $x_{0}^{*}=\left(V_{0}^{*}, K_{0}^{*}\right)$, as long as $x_{j}^{*} \in D_{j}\left(0, \hat{g}_{0}\right)$. For $m^{2}=0$, the continuity interval in Theorem 6.3 is $\tilde{\mathbb{I}}_{j+1}(0)=\left[0, L^{-2 j}\right]$ (recall (5.12) and $(\underline{6.18})$ ). Since $\check{V}_{0}\left(m^{2}, g_{0}\right) \rightarrow V_{0}^{*}$, it follows by induction and the continuity of the 
$\operatorname{maps} \rho_{j}=\check{R}_{j+1}: \mathcal{W}_{j}^{0}\left(0, \hat{g}_{0}\right) \times\left[0, L^{-2 j}\right] \rightarrow \mathcal{V}$ and $\psi_{j}=\check{K}_{j+1}: \mathcal{W}_{j}^{0}\left(0, \hat{g}_{0}\right) \times\left[0, L^{-2 j}\right] \rightarrow \mathcal{W}_{j+1}^{0}\left(0, \hat{g}_{0}\right)$, that $x_{j}\left(m^{2}, g_{0}\right) \rightarrow x_{j}^{*}$ in $\mathcal{V} \times \mathcal{W}_{j}^{0}\left(0, \hat{g}_{0}\right)$. By an analogous induction, using continuity of $\bar{V}_{j}$ and $\psi_{j}$, it follows that $\bar{K}_{j}\left(m^{2}, g_{0}\right) \rightarrow \bar{K}_{j}\left(0, \hat{g}_{0}\right)$ in $\mathcal{W}_{j}^{0}\left(0, \hat{g}_{0}\right)$. Since $\chi_{j}\left(m^{2}\right) \rightarrow \chi_{j}(0)=1$, we can now take the limit of (7.35)-(7.38) along the sequence $\left(m^{2}, g_{0}\right) \rightarrow\left(0, \hat{g}_{0}\right)$ and obtain, with (7.28),

$$
\begin{aligned}
& \left|g_{j}^{*}-\bar{g}_{j}\left(0, \hat{g}_{0}\right)\right| \leq b h \bar{g}_{j}\left(0, \hat{g}_{0}\right)^{2}\left|\log \bar{g}_{j}\left(0, \hat{g}_{0}\right)\right|, \\
& \left|\mu_{j}^{*}-\bar{\mu}_{j}\left(0, \hat{g}_{0}\right)\right| \leq b h \bar{g}_{j}\left(0, \hat{g}_{0}\right)^{2}\left|\log \bar{g}_{j}\left(0, \hat{g}_{0}\right)\right| \text {, } \\
& \left|z_{j}^{*}-\bar{z}_{j}\left(0, \hat{g}_{0}\right)\right| \leq b h \bar{g}_{j}\left(0, \hat{g}_{0}\right)^{2}\left|\log \bar{g}_{j}\left(0, \hat{g}_{0}\right)\right|, \\
& \left\|K_{j}^{*}-\bar{K}_{j}\left(0, \hat{g}_{0}\right)\right\|_{\mathcal{W}_{j}^{0}\left(0, \hat{g}_{0}\right)} \leq b\left(a-a_{*}\right) \bar{g}_{j}\left(0, \hat{g}_{0}\right)^{3} \text {. }
\end{aligned}
$$

This shows inductively that $x_{j}^{*}$ does remain in $D_{j}\left(0, \hat{g}_{0}\right)$ for all $j$, so the above inductions can be carried out indefinitely. By the uniqueness assertion of Theorem $7.2, x_{j}^{*}=\breve{x}_{j}\left(0, \hat{g}_{0}\right)$. In particular, $V_{0}^{*}=\check{V}_{0}\left(0, \hat{g}_{0}\right)$, so $\check{V}_{0}\left(m^{2}, g_{0}\right) \rightarrow \check{V}_{0}\left(0, \hat{g}_{0}\right)$ as $\left(m^{2}, g_{0}\right) \rightarrow\left(0, \hat{g}_{0}\right)$. This shows that $z_{0}^{c}, \mu_{0}^{c}$ are continuous as $m^{2} \downarrow 0$, as claimed.

\section{Proof of main result}

It is shown in Section 4 that the main result, Theorem 1.1, is a consequence of Theorem 4.1. We now prove Theorem 4.1. Functions $z_{0}^{c}$ and $\nu_{0}^{c}=\mu_{0}^{c}$ with the regularity properties required by Theorem 4.1 were constructed in Proposition 17.1. We now show that there exists $\delta>0$ such that for $m^{2}, g_{0}, \hat{g}_{0} \in(0, \delta)$,

$$
\begin{aligned}
\hat{\chi}\left(m^{2}, g_{0}, \nu_{0}^{c}\left(m^{2}, g_{0}\right), z_{0}^{c}\left(m^{2}, g_{0}\right)\right) & =\frac{1}{m^{2}}, \\
\frac{\partial \hat{\chi}}{\partial \nu_{0}}\left(m^{2}, g_{0}, \nu_{0}^{c}\left(m^{2}, g_{0}\right), z_{0}^{c}\left(m^{2}, g_{0}\right)\right) & \sim-\frac{1}{m^{4}} \frac{c\left(\hat{g}_{0}\right)}{\left(\hat{g}_{0} \mathrm{~B}_{m^{2}}\right)^{\gamma}} \quad \text { as }\left(m^{2}, g_{0}\right) \rightarrow\left(0, \hat{g}_{0}\right),
\end{aligned}
$$

with $c\left(g_{0}\right)$ continuous and equal to $1+O\left(g_{0}\right)$. This will complete the proof of Theorem 4.1. From this, an elementary analysis yields also Theorem 1.2 .

\subsection{Estimates for finite volume}

Let $\left(z_{0}^{c}\left(m^{2}, g_{0}\right), \mu_{0}^{c}\left(m^{2}, g_{0}\right)\right)$ be the functions of Proposition 7.1 , let

$$
V_{0}^{c}\left(m^{2}, g_{0}\right)=\left(g_{0}, z_{0}^{c}\left(m^{2}, g_{0}\right), \mu_{0}^{c}\left(m^{2}, g_{0}\right)\right),
$$

and let $V_{j}$ be the sequence determined by this initial condition in Proposition 7.1, Proposition 7.1 considered the the infinite volume flow, and we now wish to consider the finite volume flow. The following proposition, which is the basis for the proof of Theorem 4.1, constructs the finite volume flow. Its $V_{j}$ component is identical to the above infinite volume sequence of $V_{j}$, though of course only $j \leq N$ is meaningful for $\Lambda_{N}$. In particular, the sequence $\left(V_{j}\right)_{0 \leq j \leq N}$ is independent of the volume, in the sense that it is the same for $\Lambda_{N}$ as it is on any $\Lambda_{N^{\prime}}$ with $N^{\prime}>N$. 
Proposition 8.1. Let $d=4, N \in \mathbb{N}, g_{0} \in(0, \delta)$ and $m^{2} \in[0, \delta)$. Let $\tilde{g}_{j}=\tilde{g}_{j}\left(m^{2}, g_{0}\right)$ and $s_{j}=$ $\left(m^{2}, \tilde{g}_{j}\right)$. Let $K_{0}=\mathbb{1}_{\varnothing}$, and let $V_{j}$ be the sequence in the infinite volume global flow determined by the initial condition (8.3). Then, with the additional proviso that for the case $j+1=N$ we restrict to $m^{2} \in\left[\delta L^{-2(N-1)}, \delta\right)$, the finite volume renormalisation group flow $\left(V_{j}, K_{j, N}\right) \mapsto\left(V_{j+1}, K_{j+1, N}\right)$ of Theorem 6.3 exists for all $j<N$, with $\left(V_{j}, K_{j}\right) \in \mathbb{D}\left(s_{j}, \Lambda_{N}\right)$. In particular,

$$
\left\|K_{j, N}\right\|_{\mathcal{W}_{j}\left(s_{j}, \Lambda_{N}\right)} \leq O\left(\chi_{j} \bar{g}_{j}^{3}\right) \quad(j \leq N),
$$

$\check{g}_{j}, g_{j}=O\left(\bar{g}_{j}\right)$, and $\check{z}_{j}, \check{\mu}_{j}, z_{j}, \mu_{j}=O\left(\chi_{j} \bar{g}_{j}\right)$.

Proof. The proof is by induction on $0 \leq j \leq N$. We write $V_{j, N}$ for the perturbative coordinate, and show in the course of the proof that it is equal to $V_{j}=V_{j, \mathbb{Z}^{d}}$ as claimed. The induction hypothesis is that (8.4) holds, that $V_{j, N}=V_{j, \mathbb{Z}^{d}}$, and that the family $\left(K_{j, \Lambda}\right)$ has Property $\left(\mathbb{Z}^{d}\right)$ discussed in Section 6.5. For $j=0$, the fact that (8.4) holds is true vacuously since $K_{0}=\mathbb{1}_{\varnothing}$, the fact that $V_{0, N}=V_{0, \mathbb{Z}^{d}}$ holds by definition, and the fact that $\left(K_{0, \Lambda}\right)$ has Property $\left(\mathbb{Z}^{d}\right)$ is again vacuous. To advance the induction, it follows from (6.25)-(6.26) that $V_{j+1, N}=V_{j+1, \mathbb{Z}^{d}}$, and that $\left(K_{j+1, \Lambda}\right)$ has Property $\left(\mathbb{Z}^{d}\right)$. By Proposition $7.1, V_{j+1, N}=V_{j+1, \mathbb{Z}^{d}}$ lies in the set appearing in the domain $\mathbb{D}_{j}\left(m^{2}, \tilde{g}_{j}, \mathbb{Z}^{d}\right)$ of (6.17), which is the same set as in the domain $\mathbb{D}_{j}\left(m^{2}, \tilde{g}_{j}, \Lambda_{N}\right)$. Together with the induction hypothesis on $K_{j, N}$, we conclude that $\left(V_{j, N}, K_{j, N}\right) \in \mathbb{D}_{j}\left(m^{2}, \tilde{g}_{j}, \Lambda\right)$. By Theorem 6.3 with $\tilde{m}^{2}=m^{2}$, this implies that (8.4) holds when $j$ is replaced by $j+1$. (The restriction on $m^{2}$ at the last scale arises from $\mathbb{I}_{N}$ of (15.12).) This completes the induction and the proof.

In particular, it follows from Proposition 8.1 and (6.20) that, with the initial choice $V_{0}=$ $V_{0}^{c}\left(m^{2}, g_{0}\right)$, and with $I_{j}=I_{j}\left(V_{j}\right)$,

$$
Z_{j}=\left(I_{j} \circ K_{j, N}\right)(\Lambda) \quad(0 \leq j \leq N) .
$$

This identity continues to hold for initial conditions $V_{0} \neq V_{0}^{c}$, as long as the right-hand side remains well-defined, which is the case in an $N$-dependent neighbourhood of $V_{0}^{c}$, by continuity. In addition to the renormalisation group flow $\left(V_{j}, K_{j}\right)$, it will be convenient to use its transformed version, defined in Section 6.6. According to (7.1), the transformed flow $\left(\check{V}_{j}, K_{j}\right)=\left(T_{j}\left(V_{j}\right), K_{j}\right)$ satisfies, for all $j<N$,

$$
\left(\check{V}_{j+1}, K_{j}\right)=\left(\bar{\varphi}_{j}\left(\check{V}_{j}\right)+\check{R}_{j+1}\left(\check{V}_{j}, K_{j}\right), \check{K}_{j+1}\left(\check{V}_{j}, K_{j}\right)\right)
$$

where, by Corollary 6.5, the maps $\check{R}_{j+1}$ and $\check{K}_{j+1}$ obey the estimates (6.23) $-(6.24)$ for $0 \leq j<N$.

\subsection{Continuous functions of the renormalisation group flow}

We now show how the regularity statements of Theorem 6.3, which apply in the local domains $\mathbb{D}(\tilde{s}) \times \tilde{\mathbb{I}}_{+}\left(\tilde{m}^{2}\right)$, imply suitable regularity statements of functions of the flow of Proposition 8.1 , on the larger domain $[0, \delta) \times(0, \delta)$. We restrict attention here to the case of finite volume $\mathbb{V}=\Lambda_{N}$.

Definition 8.2. (i) A map $\left(V, K, m^{2}\right) \mapsto F\left(V, K, m^{2}\right)$ acting on a subset of $\mathcal{V} \times \mathcal{K}_{j} \times[0, \delta)$ with values in a Banach space $E$ is a continuous function of the renormalisation group coordinates at scale- $j$, if its domain includes $\mathbb{D}_{j}\left(\tilde{s}_{j}\right) \times \tilde{\mathbb{I}}_{j+1}\left(\tilde{m}^{2}\right)$ for all $\tilde{s}_{0} \in[0, \delta) \times(0, \delta)$, and if its restriction 
to the domain $\mathbb{D}_{j}\left(\tilde{s}_{j}\right) \times \tilde{\mathbb{I}}_{j+1}\left(\tilde{m}^{2}\right)$ is continuous as a map $F: \mathbb{D}_{j}\left(\tilde{s}_{j}\right) \times \tilde{\mathbb{I}}_{j+1}\left(\tilde{m}^{2}\right) \rightarrow E$, for all $\tilde{s}_{0} \in[0, \delta) \times(0, \delta)$. We also say that $F$ is a $C^{0}$ map of the renormalisation group coordinates.

(ii) For $k \in \mathbb{N}$, a map $F$ is a $C^{k}$ map of the renormalisation group coordinates at scale- $j$, if it is a $C^{0}$ map of the renormalisation group coordinates, its restrictions to the domains $\mathbb{D}_{j}\left(\tilde{s}_{j}\right) \times \tilde{\mathbb{I}}_{j+1}\left(\tilde{m}^{2}\right)$ are $k$-times continuously Fréchet differentiable in $(V, K)$, and every Fréchet derivative in $(V, K)$, when applied as a multilinear map to directions $\dot{V}$ in $\mathcal{V}^{p}$ and $\dot{K}$ in $\mathcal{W}^{q}$, is jointly continuous in all arguments, $m^{2}, V, K, \dot{V}, \dot{K}$.

Two examples of $C^{k}$ maps of the renormalisation group coordinates are $F\left(V, K, m^{2}\right)=V$, and the map $R_{+}$of Theorem 6.3. The map $K_{+}$is a not a $C^{k}$ map in the above sense, since it does not act with a common target space $E$ (its image space $\mathcal{W}_{+}$depends on $\tilde{s}$ ). In all our applications, $E$ is finite-dimensional.

For $s_{0}=\left(m^{2}, g_{0}\right) \in[0, \delta) \times(0, \delta)$, recall the definition of $V_{0}^{c}\left(s_{0}\right)$ from (8.3). Let $\left(V_{j}, K_{j}\right)=$ $\left(V_{j}\left(m^{2}, V_{0}\right), K_{j}\left(m^{2}, V_{0}\right)\right)$ be the flow of $\left(V_{+}, K_{+}\right)$with initial condition $V_{0}$ (not necessarily equal to $V_{0}^{c}$ ) and mass parameter $m^{2}$. By Proposition 8.1, this flow exists for all $j<N$ if $V_{0}=V_{0}^{c}$, and also exist for $j=N$ if $m^{2} \in\left[\delta L^{-2(N-1)}, \delta\right)$. Given a $C^{k}$ map $F$ of the renormalisation group coordinates at scale- $j$, let $D_{V_{0}}^{k} F\left(s_{0}\right)$ denote the $k^{\text {th }}$ derivative of $F\left(V_{j}\left(m^{2}, V_{0}\right), K_{j}\left(m^{2}, V_{0}\right), m^{2}\right)$ with respect to the initial condition $V_{0}$, evaluated at $V_{0}=V_{0}^{c}(s)$. The following proposition shows that $D_{V_{0}}^{k} F$ exists and is continuous in $s_{0}$.

Proposition 8.3. Let $j<N(\mathbb{V}), k \in \mathbb{N}_{0}$, and let $F$ be a $C^{k}$ map of the renormalisation group coordinates at scale- $j$. Then, for every $p \leq k$, all $s_{0} \in[0, \delta) \times(0, \delta)$, the derivative $D_{V_{0}}^{p} F\left(s_{0}\right)$ exists, and

$$
s_{0} \mapsto D_{V_{0}}^{p} F\left(s_{0}\right) \text { is a continuous map }[0, \delta) \times(0, \delta) \rightarrow L^{p}(\mathcal{V}, E),
$$

where $L^{p}(\mathcal{V}, E)$ is the space of p-linear maps from $\mathcal{V}$ to $E$ with the operator norm.

In particular, with $k=0$, continuous maps of the renormalisation group coordinates at scale- $j$, in the sense of Definition 8.2 , induce continuous functions in the ordinary sense of the parameters $\left(m^{2}, g_{0}\right)$ of the renormalisation group flow.

To give an example of an application of Proposition 8.3, it follows from Corollary 6.5 that

$$
\check{g}_{j+1}=\check{g}_{j}-\beta_{j} \check{g}_{j}^{2}+r_{j} \quad \text { with } \quad r_{j}=O\left(\chi_{j} \check{g}_{j}^{3}\right)
$$

It also follows from Theorem 6.3 that the function $F\left(V_{j}, K_{j}, m^{2}\right)=g_{j+1}$ is a $C^{\infty}$ map of the renormalisation group coordinates at scale- $j$. Therefore, by Proposition 8.3, we conclude the continuity of $g_{j}$ in $\left(m^{2}, g_{0}\right) \in[0, \delta) \times(0, \delta)$, for all $j \leq N$. Furthermore, since $g_{j}=O\left(g_{0}\right)$, the continuity extends to $\left(m^{2}, g_{0}\right) \in[0, \delta)^{2}$. From (8.8) and the continuity of $\beta_{j}$ in $m^{2} \in[0, \delta)$, we therefore also have continuity of $r_{j}=r_{j}\left(m^{2}, g_{0}\right)$ in $\left(m^{2}, g_{0}\right) \in[0, \delta)^{2}$, for all $j<N$.

Proof of Proposition 8.3. Let $\tilde{s}_{0}=\left(\tilde{m}^{2}, g_{0}\right) \in[0, \delta) \times(0, \delta)$, and set $\tilde{s}_{j}=\left(\tilde{m}^{2}, \tilde{g}_{j}\left(\tilde{m}^{2}, \tilde{g}_{0}\right)\right)$. For $s_{0} \in[0, \delta) \times(0, \delta)$ sufficiently close to $\tilde{s}_{0}$, we write $\left[D_{V_{0}}^{k}\left(V_{j}, K_{j}\right)\right]\left(s_{0}\right)$ for the $k^{\text {th }}$ derivative of $\left(V_{j}\left(m^{2}, V_{0}\right), K_{j}\left(m^{2}, V_{0}\right)\right)$ with respect to the initial condition $V_{0}$, evaluated at $V_{0}=V_{0}^{c}\left(s_{0}\right)$ of (8.3) and with derivative taken in $\mathcal{V} \times \mathcal{W}_{j}\left(\tilde{s}_{j}\right)$. In particular, $\left[D_{V_{0}}^{0}\left(V_{j}, K_{j}\right)\right]\left(s_{0}\right)=\left(V_{j}\left(s_{0}\right), K_{j}\left(s_{0}\right)\right)$ is the renormalisation group flow with initial condition determined by $s_{0}$ in Proposition 8.1 . To see that the derivatives exist, we claim that for every $\tilde{s}_{0}=\left(\tilde{m}^{2}, \tilde{g}_{0}\right) \in[0, \delta) \times(0, \delta), k \in \mathbb{N}_{0}$, and $j<N$,

$$
s_{0} \mapsto D_{V_{0}}^{k}\left(V_{j}, K_{j}\right) \text { is continuous in } L^{k}\left(\mathcal{V}, \mathcal{V} \times \mathcal{W}_{j}\left(\tilde{s}_{j}\right)\right) \text { as } s_{0} \rightarrow \tilde{s}_{0}
$$


We prove (8.9) as follows. First, we know from Proposition 8.1 that $\left(V_{j}\left(\tilde{s}_{0}\right), K_{j}\left(\tilde{s}_{0}\right)\right) \in \mathbb{D}_{j}\left(\tilde{s}_{j}\right)$, and that $z_{0}^{c}, \mu_{0}^{c}$ are continuous. Also, the maps $\left(V_{+}, K_{+}\right)$are continuous by Theorem 6.3 . Therefore, there exist neighbourhoods $N_{j}=N_{j}\left(\tilde{s}_{0}\right)$ of $\tilde{s}_{0}$ with $\left(V_{j}\left(s_{0}\right), K_{j}\left(s_{0}\right)\right) \in \mathbb{D}_{j}\left(\tilde{s}_{j}\right)$ for $s_{0} \in N_{j}$, and such that $\left(V_{j}\left(s_{0}\right), K_{j}\left(s_{0}\right)\right)$ is continuous in $s_{0} \in N_{j}$ as a map with values in $\mathcal{V} \times \mathcal{W}_{j}\left(\tilde{s}_{j}\right)$. In particular, for $j<N$, the case $k=0$ of $\left(V_{j}, K_{j}\right)$ as $s_{0} \rightarrow \tilde{s}_{0}$ in (8.9) follows.

For $k>0$, the existence and continuity of $D_{V_{0}}^{k}\left(V_{j}, K_{j}\right)$ in (8.9) is a consequence of the continuity the derivatives of the maps $V_{+}, K_{+}$in $\mathbb{D}_{j}\left(\tilde{s}_{j}\right) \times \tilde{\mathbb{I}}_{j+1}\left(\tilde{m}^{2}\right)$ provided by Theorem 6.3. By induction, we assume that the claim holds for some $j<N$. It is vacuous for $j=0$. To advance the induction, we consider, e.g., $D_{V_{0}} K_{j+1}$. By the chain rule,

$$
D_{V_{0}} K_{j+1}=D_{V} K_{j+1}\left(V_{j}, K_{j}, m^{2}\right) D_{V_{0}} V_{j}+D_{K} K_{j+1}\left(V_{j}, K_{j}, m^{2}\right) D_{V_{0}} K_{j} .
$$

By the inductive assumption, each of $V_{j}, D_{V_{0}} V_{j}, K_{j}, D_{V_{0}} K_{j}$ is continuous as $s_{0} \rightarrow \tilde{s}_{0}$. The continuity

statement of Theorem 6.3 advances the induction. An analogous argument applies to $D_{V_{0}} V_{j+1}$, and also to all higher derivatives. This completes the proof of (8.9)).

The assumption that $F$ is a continuous map of the renormalisation group coordinates and (8.9) imply that $F\left(V_{j}\left(s_{0}\right), K_{j}\left(s_{0}\right), m^{2}\right) \rightarrow F\left(V_{j}\left(\tilde{s}_{0}\right), K_{j}\left(\tilde{s}_{0}\right), \tilde{m}^{2}\right)$ as $s_{0} \rightarrow \tilde{s}_{0}$. Since $\tilde{s}_{0} \in[0, \delta) \times(0, \delta)$ is arbitrary, the claim (8.7) follows for $k=0$. Similarly, for $k>0$, the proof of (8.7) follows from the chain rule and (8.9).

\subsection{Susceptibility and renormalisation group flow}

As the first step in the analysis of the susceptibility, we express $\hat{\chi}_{N}$ in terms of the renormalisation group coordinates. Let $N \in \mathbb{N}$ and let $m^{2} \in\left[\delta L^{-2(N-1)}, \delta\right)$. Recall from (4.26) that

$$
\hat{\chi}_{N}=\frac{1}{m^{2}}+\frac{1}{m^{4}} \frac{1}{|\Lambda|} D^{2} Z_{N}^{0}(0,0 ; 1,1)
$$

with $Z_{N}^{0}$ the degree-0 part of the form $Z_{N}=\mathbb{E}_{C} \theta Z_{0}$. Let $\left(V_{j}, K_{j, N}\right)$ be the finite volume flow given by Proposition 8.1. With the abbreviation $K_{N}=K_{N, N}$, it follows from (8.5), together with the fact that $\mathcal{B}_{N}\left(\Lambda_{N}\right)$ consists only of $\varnothing, \Lambda$, that

$$
Z_{N}=\left(I_{N} \circ K_{N}\right)(\Lambda)=I_{N}(\Lambda)+K_{N}(\Lambda)
$$

Therefore,

$$
\hat{\chi}_{N}=\frac{1}{m^{2}}+\frac{1}{m^{4}} \frac{1}{|\Lambda|} D^{2} I_{N}^{0}(0,0 ; 1,1)+\frac{1}{m^{4}} \frac{1}{|\Lambda|} D^{2} K_{N}^{0}(0,0 ; 1,1) .
$$

By (15.22),$I_{N}(\Lambda)=e^{-V_{N}(\Lambda)}\left(1+W_{N}(\Lambda)\right)$, so $I_{N}^{0}(\Lambda)=e^{-V_{N}^{0}(\Lambda)}\left(1+W_{N}^{0}(\Lambda)\right)$ where $V_{N}^{0}(\Lambda)$ and $W_{N}^{0}(\Lambda)$ are the degree-0 parts of the forms $V_{N}(\Lambda)$ and $W_{N}(\Lambda)$, respectively. Thus

$$
D^{2} I_{N}^{0}(\Lambda ; 0,0 ; 1,1)=D^{2} e^{-V_{N}^{0}}(\Lambda ; 0,0 ; 1,1)+D^{2} W_{N}^{0}(\Lambda ; 0,0 ; 1,1)
$$

since cross-terms cancel when $\phi=0$ because $W_{N}^{0}$ is a polynomial in $\phi$ with no monomials of degree below two. The first term on the right-hand side of (8.14) can be evaluated by direct calculation, using (5.21) and (3.8)-(3.9), to give

$$
D^{2} e^{-V_{N}^{0}}(\Lambda ; 0,0 ; 1,1)=-\sum_{x, y} \nu_{N} \delta_{x y} 1_{x} 1_{y}-\sum_{x, y} z_{N}\left(-\Delta_{x y}\right) 1_{x} 1_{y}=-\nu_{N}|\Lambda|,
$$


since the quartic term $\tau^{2}$ does not contribute, and $\Delta 1=0$. This gives the identity

$$
\hat{\chi}_{N}=\frac{1}{m^{2}}-\frac{\nu_{N}}{m^{4}}+\frac{1}{m^{4}} \frac{1}{|\Lambda|} D^{2} W_{N}^{0}(0,0 ; 1,1)+\frac{1}{m^{4}} \frac{1}{|\Lambda|} D^{2} K_{N}^{0}(0,0 ; 1,1) .
$$

By Proposition [7.1, $\nu_{N}=O\left(\chi_{N} L^{-2 N} \bar{g}_{N}\right)$, which converges exponentially to 0 , so the second term in (8.16) does not contribute to the limit $N \rightarrow \infty$. We will prove (8.1) by showing that the two rightmost terms in (8.16) are even smaller, by factors $O\left(\bar{g}_{N}\right)$ and $O\left(\bar{g}_{N}^{2}\right)$, respectively, so that the limit is $m^{-2}$ as desired.

For (8.2), the constant term $m^{-2}$ in (8.16) does not contribute because its derivative is zero. We denote the derivative of $\left(V_{N}, K_{N}\right)$ with respect to $\nu_{0}$, evaluated at $V_{0}=V_{0}^{c}$, by $V_{N}^{\prime}=\left(g_{N}^{\prime}, z_{N}^{\prime}, \mu_{N}^{\prime}\right)$ and $K_{N}^{\prime}$. We will show that $-m^{-4} \nu_{N}^{\prime}$ in the derivative of (8.2) is asymptotic to the right-hand side of (8.2), and that the derivatives of the last two terms in (8.16) are again smaller by $O\left(\bar{g}_{N}\right)$ and $O\left(\bar{g}_{N}^{2}\right)$, respectively. To execute this strategy, we use the following sequence of lemmas. The bubble diagram $\mathrm{B}_{m^{2}}=8 B_{m^{2}}$ defined in (1.8), with its logarithmic divergence in $d=4$, enters via Lemmas 8.4 8.5. The power $\gamma=\frac{1}{4}$ for the logarithmic correction to the susceptibility in $d=4$ arises in Lemma 8.6. Lemmas 8.5 8.6 involve extensions of arguments used in [7].

Lemma 8.4. For $m^{2}>0$,

$$
\sum_{j=0}^{\infty} \beta_{j}=\mathrm{B}_{m^{2}}
$$

Proof. By the definition of $\beta_{j}$ in (6.5),

$$
\sum_{j=0}^{k-1} \beta_{j}=8 \sum_{x \in \mathbb{Z}^{d}} w_{k}(x)^{2}
$$

since the left-hand side is a telescoping sum. Since the terms in the covariance decomposition are positive definite, the Fourier transforms satisfy $0 \leq \hat{w}_{k} \leq \hat{C}$, where $C=\left(-\Delta_{\mathbb{Z}^{d}}+m^{2}\right)^{-1}$ is the Green function on $\mathbb{Z}^{d}$. By the Parseval relation, the dominated converge theorem, and (1.8),

$$
\lim _{k \rightarrow \infty} \sum_{x \in \mathbb{Z}^{d}} w_{k}(x)^{2}=\lim _{k \rightarrow \infty} \int_{[-\pi, \pi]^{d}} \hat{w}_{k}(p)^{2} \frac{d p}{(2 \pi)^{d}}=\int_{[-\pi, \pi]^{d}} \hat{C}(p)^{2} \frac{d p}{(2 \pi)^{d}}=B_{m^{2}} .
$$

Since $\mathrm{B}_{m^{2}}=8 B_{m^{2}}$, the proof is complete.

For the subsequent analysis, we note the following facts. First, in the upper bounds of (6.23)(6.24), $\tilde{g}_{j}$ can be replaced by $\check{g}_{j}$ or $\bar{g}_{j}$ due to Lemma 7.4, e.g.,

$$
\check{g}_{j+1}=\check{g}_{j}-\beta_{j} \check{g}_{j}^{2}+r_{j} \quad \text { with } \quad r_{j}=O\left(\chi_{j} \check{g}_{j}^{3}\right)=O\left(\bar{g}_{j}^{3}\right)
$$

As argued below (8.8),$r_{j}=r_{j}\left(m^{2}, g_{0}\right)$ is continuous on $[0, \delta)^{2}$. Secondly, it follows from (8.20) that for any continuously differentiable function $\psi:(0, \infty) \rightarrow \mathbb{R}$,

$$
\sum_{l=j}^{k}\left(\beta_{l} \check{g}_{l}^{2}-r_{l}\right) \psi\left(\check{g}_{l}\right)=\int_{\check{g}_{k+1}}^{\check{g}_{j}} \psi(t) d t+O\left(\int_{\check{g}_{k+1}}^{\check{g}_{j}} t^{2}\left|\psi^{\prime}(t)\right| d t\right) .
$$


The formula (8.21) is proved in [7, (2.12)] for the special case $r_{j}=0$, but the same proof applies when $r_{j}=O\left(\chi_{j} \check{g}_{j}^{3}\right)$. We also use the fact, proved in [7, Lemma 2.1(ii)], that

$$
\sum_{l=j}^{k} \chi_{l} \bar{g}_{l}^{n} \leq C_{n} \begin{cases}\left|\log \bar{g}_{k}\right| & (n=1) \\ \chi_{j} \bar{g}_{j}^{n-1} & (n>1)\end{cases}
$$

Lemma 8.5. Let $d=4$. For $\left(m^{2}, g_{0}\right) \in(0, \delta)^{2}$, the limit $\check{g}_{\infty}=\lim _{j \rightarrow \infty} \check{g}_{j}$ exists, is continuous in $\left(m^{2}, g_{0}\right)$, and extends continuously to $[0, \delta)^{2}$. For $\hat{g}_{0} \in(0, \delta)$, as $m^{2} \downarrow 0$ and $g_{0} \rightarrow \hat{g}_{0}$,

$$
\check{g}_{\infty} \sim \frac{1}{\mathrm{~B}_{m^{2}}} .
$$

Proof. As mentioned above, the remainder $r_{j}=r_{j}\left(m^{2}, g_{0}\right)$ in (8.20) is a continuous function of $\left(m^{2}, g_{0}\right) \in[0, \delta)^{2}$. The solution to the recursion (8.20) is given by

$$
\check{g}_{j+1}=g_{0} \prod_{k=0}^{j}\left(1-\beta_{k} \check{g}_{k}-\check{g}_{k}^{-1} r_{k}\right)=g_{0} \exp \left(\sum_{k=0}^{j} \log \left(1-\beta_{k} \check{g}_{k}-O\left(\chi_{k} \bar{g}_{k}^{2}\right)\right)\right) .
$$

By the dominated convergence theorem and (8.22), the limit

$$
\check{g}_{\infty}=\lim _{j \rightarrow \infty} \check{g}_{j}=g_{0} \exp \left(\sum_{k=0}^{\infty} \log \left(1-\beta_{k} \check{g}_{k}+O\left(\chi_{k} \check{g}_{k}^{2}\right)\right)\right)
$$

exists and is continuous in $\left(m^{2}, g_{0}\right) \in[0, \delta)^{2}$. It remains to prove (8.23). For this, we set $\psi(t)=t^{-2}$ in (8.21), and use that $\sum_{l=0}^{k-1} r_{l} \check{g}_{l}^{-2}=O\left(\sum_{l=0}^{k-1} \chi_{l} \bar{g}_{l}\right)=O\left(\log \check{g}_{l}\right)$ by (8.22), to obtain

$$
\check{g}_{k}^{-1}=g_{0}^{-1}+\sum_{j=0}^{k-1} \beta_{j}+O\left(\log \check{g}_{k}\right) .
$$

Therefore, by Lemma 8.4, if $\left(m^{2}, g_{0}\right) \in(0, \delta)^{2}$ then

$$
\check{g}_{\infty}^{-1}+O\left(\left|\log \check{g}_{\infty}\right|\right)=g_{0}^{-1}+\mathrm{B}_{m^{2}} .
$$

In particular $\check{g}_{\infty} \rightarrow 0$ as $m^{2} \downarrow 0$ or $g_{0} \downarrow 0$, and $\check{g}_{\infty}$ is right-continuous also at $m=0$ and $g_{0}=0$. Finally, (8.23) follows from (8.27). This completes the proof.

In the proof of the following lemma, we use the fact that

$$
\prod_{k=j}^{l}\left(1-\gamma \beta_{k} \check{g}_{k}\right)^{-1}=\left(\frac{\check{g}_{j}}{\check{g}_{l+1}}\right)^{\gamma}\left(c_{j}+O\left(\chi_{l} \bar{g}_{l}\right)\right),
$$

with $c_{j}=1+O\left(\chi_{j} \bar{g}_{j}\right)$ a continuous function of $\left(m^{2}, g_{0}\right) \in[0, \delta)^{2}$. The product formula (8.28) is a consequence of the recursion relation (8.20); its proof is identical to that of [7, Lemma 2.1(iii-a)] where the same statement is proved with $r_{j}=0$. As in [7, Lemma 2.1(iii-a)], the continuity of $c_{j}$ follows from the continuity of $\beta_{j}$ and $\check{g}_{j}$ (the latter was established below (8.8)). In the next lemma, we use primes to denote derivatives with respect to the initial value $\nu_{0}^{c}=\mu_{0}^{c}$. 
Lemma 8.6. Let $d=4$. Let $\left(m^{2}, g_{0}\right) \in[0, \delta) \times(0, \delta),\left(z_{0}, \mu_{0}\right)=\left(z_{0}^{c}, \mu_{0}^{c}\right)$, and $s_{j}=\left(m^{2}, \tilde{g}_{j}\left(m^{2}, g_{0}\right)\right)$. There exists a continuous function $c:[0, \delta)^{2} \rightarrow \mathbb{R}$, which satisfies $c\left(m^{2}, g_{0}\right)=1+O\left(g_{0}\right)$, such that for all $j \in \mathbb{N}_{0}$ :

$$
\check{\mu}_{j}^{\prime}=L^{2 j}\left(\frac{\check{g}_{j}}{g_{0}}\right)^{\gamma}\left(c\left(m^{2}, g_{0}\right)+O\left(\chi_{j} \check{g}_{j}\right)\right), \quad \check{g}_{j}^{\prime}=O\left(\check{\mu}_{j}^{\prime} \check{g}_{j}^{2}\right), \quad \check{z}_{j}^{\prime}=O\left(\chi_{j} \check{\mu}_{j}^{\prime} \check{g}_{j}^{2}\right)
$$

Also, for $N \in \mathbb{N}_{0}$ and $\left(m^{2}, g_{0}\right) \in\left[\delta L^{-2(N-1)}, \delta\right) \times(0, \delta)$,

$$
\left\|K_{j}^{\prime}\right\|_{\mathcal{W}_{j}\left(s_{j}, \Lambda_{N}\right)}=O\left(\chi_{j} \check{\mu}_{j}^{\prime} \check{g}_{j}^{2}\right)
$$

with $K_{j}$ the sequence of Proposition 8.1 corresponding to $\left(m^{2}, g_{0}\right)$.

Proof. For $s_{0}=\left(m^{2}, g_{0}\right) \in[0, \delta)^{2}$, let $V_{j}=V_{j}\left(s_{0}\right)$ be the infinite sequence of Proposition 8.1 with initial condition specified by $s_{0}$. Let $\check{V}_{j}=T_{j}\left(V_{j}\right)=\left(\check{g}_{j}, \check{z}_{j}, \check{\mu}_{j}\right)$ be the transformed flow. For $m^{2} \in\left[\delta L^{-2(N-1)}, \delta\right)$, let $K_{j}=K_{j}\left(s_{0}\right)$ be the sequence given by Proposition 8.1 , so that $\left(\check{V}_{j}, K_{j}\right)$ is a finite volume flow of $\left(\check{V}_{+}, \check{K}_{+}\right)$, as in (8.6). (In fact, $K_{j}$ is defined for all $m^{2} \in[0, \delta)$ if $j<N$.) Let $\left(\check{V}_{j}^{\prime}, K_{j}^{\prime}\right)$ denote the sequence of derivatives along this solution, with respect to the initial condition $\check{\mu}_{0}=\mu_{0}$, and with the derivative $K_{j}^{\prime}$ taken in the space $\mathcal{W}_{j}\left(s_{j}\right)=\mathcal{W}_{j}\left(s_{j}, \Lambda_{N}\right)$. Since the sequence $V_{j}$ is independent of $\Lambda_{N}$ by Proposition 8.1, the sequence $\check{V}_{j}^{\prime}$ is also independent of $N$ and can be extended inductively to all $j \in \mathbb{N}_{0}$ by choosing $N>j$.

We define $\Pi_{j}=\Pi_{j}\left(m^{2}, g_{0}\right)$ by

$$
\Pi_{j}=L^{2 j} \prod_{l=0}^{j-1}\left(1-\gamma \beta_{l} \check{g}_{l}\right)
$$

By (8.28), there is a continuous function $\Gamma_{\infty}\left(m^{2}, g_{0}\right)=O\left(g_{0}\right)$ such that

$$
\Pi_{j}=L^{2 j}\left(\frac{\check{g}_{j}}{\check{g}_{0}}\right)^{\gamma}\left(1+\Gamma_{\infty}\left(m^{2}, g_{0}\right)+O\left(\chi_{j} \check{g}_{j}\right)\right)
$$

We also define $\Sigma_{j}=\Sigma_{j}\left(m^{2}, g_{0}\right)$ by

$$
\check{\mu}_{j}^{\prime}=\Pi_{j}\left(1+\Sigma_{j}\right) \quad(j \geq 0), \quad \Sigma_{-1}=0 .
$$

We make the inductive assumption that for $j<N$ there exist $M_{1} \gg M_{2} \gg 1$ such that

$$
\left|\Sigma_{j}-\Sigma_{j-1}\right| \leq O\left(M_{1}+M_{2}\right) \chi_{j} \bar{g}_{j}^{2}, \quad\left|\check{g}_{j}^{\prime}\right|,\left|\check{z}_{j}^{\prime}\right| \leq M_{1} \chi_{j} \Pi_{j} \bar{g}_{j}^{2}, \quad\left\|K_{j}^{\prime}\right\|_{\mathcal{W}_{j}\left(s_{j}, \mathbb{V}\right)} \leq M_{2} \chi_{j} \Pi_{j} \bar{g}_{j}^{2} .
$$

Since $\left(\check{g}_{0}^{\prime}, \check{z}_{0}^{\prime}, \check{\mu}_{0}^{\prime}, K_{0}^{\prime}\right)=(0,0,1,0)$, the inductive assumption (8.34) is true for $j=0$. To advance the induction, we begin by applying (8.32) $(8.33)$ to conclude that, if $L \gg 1$, if $\Omega \leq L$, and if $g_{0}$ is sufficiently small, then

$$
\left|\check{\mu}_{j}^{\prime}\right| \leq 2 \Pi_{j}, \quad \chi_{j} \Pi_{j} \bar{g}_{j}^{2} \leq \frac{1}{2} \chi_{j+1} \Pi_{j+1} \bar{g}_{j+1}^{2} .
$$

We also use differentiated versions of the flow equation (8.6). By the chain rule, with $F=\check{R}_{j+1}$ or $F=\check{K}_{j+1}$,

$$
F^{\prime}\left(\check{V}_{j}, K_{j}\right)=D_{\check{V}} F\left(\check{V}_{j}, K_{j}\right) \check{V}_{j}^{\prime}+D_{K} F\left(\check{V}_{j}, K_{j}\right) K_{j}^{\prime}
$$


By the estimates of Theorem 6.3 (which apply as discussed below (8.6)) and by (8.34), this gives

$$
\begin{aligned}
\left\|D_{V} F\left(\check{V}_{j}, K_{j}\right) \check{V}_{j}^{\prime}\right\| & \leq O\left(\chi_{j} \bar{g}_{j}^{2}\right)\left(M_{1} \bar{g}_{j}^{2}+2\right) \Pi_{j} \leq O\left(\chi_{j} \Pi_{j} \bar{g}_{j}^{2}\right), \\
\left\|D_{K} \check{R}_{j+1}\left(\check{V}_{j}, K_{j}\right) K_{j}^{\prime}\right\| & \leq O\left(M_{2}\right) \chi_{j} \Pi_{j} \bar{g}_{j}^{2} \\
\left\|D_{K} \check{K}_{j+1}\left(\check{V}_{j}, K_{j}\right) K_{j}^{\prime}\right\| & \leq M_{2} \chi_{j} \Pi_{j} \bar{g}_{j}^{2},
\end{aligned}
$$

where the norms on the left-hand sides are those of the appropriate $\mathcal{V}, \mathcal{W}\left(s_{j}\right)$ spaces. This implies, for $M_{2} \gg 1$,

$$
\left\|\check{R}_{j+1}^{\prime}\left(\check{V}_{j}, K_{j}\right)\right\| \leq O\left(M_{2}\right) \chi_{j} \Pi_{j} \bar{g}_{j}^{2}, \quad\left\|\check{K}_{j+1}^{\prime}\left(\check{V}_{j}, K_{j}\right)\right\| \leq 2 M_{2} \chi_{j} \Pi_{j} \bar{g}_{j}^{2}
$$

For $\check{\mu}$, the induction is advanced using the recursion (8.6) with (6.4), (8.40), and

$$
\left(\eta_{j} \check{g}_{j}\right)^{\prime},\left(\xi_{j} \check{g}_{j}^{2}\right)^{\prime},\left(\check{g}_{j} \check{z}_{j}\right)^{\prime}=O\left(M_{1} \chi_{j} \Pi_{j} \bar{g}_{j}^{2}\right)
$$

It follows that

$$
\begin{aligned}
\check{\mu}_{j+1}^{\prime} & =L^{2} \check{\mu}_{j}^{\prime}\left(1-\gamma \beta_{j} \check{g}_{j}\right)+O\left(\left(M_{1}+M_{2}\right) \chi_{j} \Pi_{j} \bar{g}_{j}^{2}\right) \\
& =\Pi_{j+1}\left(1+\Sigma_{j}\right)+O\left(\left(M_{1}+M_{2}\right) \chi_{j+1} \Pi_{j+1} \bar{g}_{j+1}^{2}\right) .
\end{aligned}
$$

This enables us to advance the induction for $\check{\mu}$, namely the first estimate of (8.34). The advancement of the induction for $\check{g}$ and $\check{z}$ is similar, as follows. We use the recursion relation (8.6) with (6.2) - (6.3) and (8.40), and choose $M_{1} \gg M_{2}$ to obtain

$$
\begin{aligned}
\left|\check{g}_{j+1}^{\prime}\right|,\left|\check{z}_{j+1}^{\prime}\right| & \leq\left(M_{1}\left(1+O\left(\bar{g}_{j}\right)\right)+O\left(M_{2}\right)\right) \chi_{j} \Pi_{j} \bar{g}_{j}^{2} \\
& \leq 2 M_{1} \chi_{j} \Pi_{j} \bar{g}_{j}^{2} \leq M_{1} \chi_{j+1} \Pi_{j+1} \bar{g}_{j+1}^{2} .
\end{aligned}
$$

This advances the induction for $\check{g}$ and $\check{z}$.

We now complete the proof, having established that (8.34) holds for all $j<N$. As discussed in the first paragraph of the proof, the bound for $\check{V}_{j}^{\prime}$ in fact holds for all $j \in \mathbb{N}_{0}$. Let $F\left(V, K, m^{2}\right)=V$. By Proposition 8.1 and Theorem 6.3, $F$ is a $C^{\infty}$ map of the renormalisation group coordinates at scale- $j$, in the sense of Definition 8.2, for all $j \in \mathbb{N}_{0}$. Therefore, by Proposition 8.3, $V_{j}^{\prime}\left(s_{0}\right)$ is continuous in $s_{0} \in[0, \delta) \times(0, \delta)$ for each $j \in \mathbb{N}_{0}$. The same is therefore true for $\check{V}_{j}^{\prime}\left(s_{0}\right)$. As a consequence, since $\Pi_{j}$ is continuous, it follows that $\Sigma_{j}\left(s_{0}\right)$ is continuous in $[0, \delta) \times(0, \delta)$, for each $j \in \mathbb{N}_{0}$. Since $\sum_{j=1}^{\infty} \chi_{j} \bar{g}_{j}^{2}=O\left(g_{0}\right)$ by (8.22), it follows from (8.34) that the limit $\Sigma_{\infty}=\lim _{j \rightarrow \infty} \Sigma_{j}=$ $\sum_{j=1}^{\infty}\left(\Sigma_{j}-\Sigma_{j-1}\right)$ exists with $\Sigma_{\infty}=O\left(g_{0}\right)$. In particular, $\Sigma_{j}$ and $\Sigma_{\infty}$ extend continuously as $g_{0} \downarrow 0$. Similarly, continuity of $\Sigma_{\infty}$ on $[0, \delta)^{2}$ follows from the dominated convergence theorem, with (6.8). Also, again by (8.22),

$$
\Sigma_{\infty}-\Sigma_{j}=O\left(\sum_{k=j+1}^{\infty} \chi_{k} \bar{g}_{j}^{2}\right)=O\left(\chi_{j} \bar{g}_{j}\right) .
$$

From (8.32)-(8.33) and (8.44), we obtain the equation for $\check{\mu}_{j}^{\prime}$ in (8.29), with $c\left(m^{2}, g_{0}\right)=(1+$ $\left.\Sigma_{\infty}\left(m^{2}, g_{0}\right)\right)\left(1+\Gamma_{\infty}\left(m^{2}, g_{0}\right)\right)$. This $c\left(m^{2}, g_{0}\right)$ is indeed continuous, since $\Sigma_{\infty}$ and $\Gamma_{\infty}$ are. With this, (8.34) implies the last two equations in (8.29) and (8.30). 
Lemmas 8.5 8.6 are stated in terms of the transformed variables. However, since $\check{g}_{j}=g_{j}+$ $O\left(g_{j}^{2}\right)$, by (6.1), Lemma 8.5 also implies $g_{\infty} \sim \check{g}_{\infty} \sim 1 / \mathrm{B}_{m^{2}}$. Moreover, we note that (8.29) remains true if $\check{\mu}_{j}^{\prime}$ is replaced by $\mu_{j}^{\prime}$. In fact, by [6, (6.93)], there exist constants $a_{j}=O(1)$ such that

$$
\check{\mu}_{j}=\mu_{j}+a_{j} \mu_{j}^{2} .
$$

Since $\mu_{j}=O\left(\chi_{j} \check{g}_{j}\right)$, by Proposition 8.1, this indeed implies

$$
\mu_{j}^{\prime}=\check{\mu}_{j}^{\prime}\left(1+O\left(\mu_{j}\right)\right)=L^{2 j}\left(\frac{\check{g}_{j}}{g_{0}}\right)^{\gamma}\left(c\left(m^{2}, g_{0}\right)+O\left(\chi_{j} \check{g}_{j}\right)\right) .
$$

In particular, setting $c\left(\hat{g}_{0}\right)=c\left(0, \hat{g}_{0}\right)$, and by Lemma 8.5 ,

$$
\lim _{N \rightarrow \infty} \nu_{N}^{\prime}=c\left(m^{2}, g_{0}\right)\left(\frac{\check{g}_{\infty}}{g_{0}}\right)^{\gamma} \sim \frac{c\left(\hat{g}_{0}\right)}{\left(\hat{g}_{0} \mathrm{~B}_{m^{2}}\right)^{\gamma}} \quad \text { as }\left(m^{2}, g_{0}\right) \rightarrow\left(0, \hat{g}_{0}\right) .
$$

Similarly, by [6, (6.91)-(6.92)],

$$
g_{j}^{\prime}=O\left(g_{j} \mu_{j}^{\prime}\right), \quad z_{j}^{\prime}=O\left(\chi_{j} g_{j} \mu_{j}^{\prime}\right) .
$$

\subsection{Proof of Theorem 4.1}

Proof of Theorem 4.1. Let $\nu_{0}^{c}=\mu_{0}^{c}$ and $z_{0}^{c}$ be the functions of Proposition 17.1, which as desired are continuous in $\left(\mathrm{m}^{2}, g_{0}\right)$ and differentiable in $g_{0}$. As discussed at the beginning of Section 8 , it suffices to show that for $\hat{\chi}$ and $\hat{\chi}^{\prime}$ evaluated at $\left(m^{2}, g_{0}, \nu_{0}^{c}, z_{0}^{c}\right)$,

$$
\hat{\chi}=\frac{1}{m^{2}}, \quad \hat{\chi}^{\prime} \sim-\frac{1}{m^{4}} \frac{c\left(\hat{g}_{0}\right)}{\left(\hat{g}_{0} \mathrm{~B}_{m^{2}}\right)^{\gamma}} \quad \text { as }\left(m^{2}, g_{0}\right) \rightarrow\left(0, \hat{g}_{0}\right),
$$

with $c$ continuous and $c\left(g_{0}\right)=1+O\left(g_{0}\right)$. We do this using the identity (8.16), which asserts that

$$
\hat{\chi}_{N}=\frac{1}{m^{2}}+\frac{1}{m^{4}}\left(-\nu_{N}+\frac{1}{|\Lambda|} D^{2} W_{N}^{0}(0,0 ; 1,1)+\frac{1}{|\Lambda|} D^{2} K_{N}^{0}(0,0 ; 1,1)\right)
$$

and is valid for initial conditions $V_{0}$ in an $N$-dependent neighbourhood of $V_{0}^{c}$, as discussed below (8.5). In particular, this allows the identity to be differentiated in $V_{0}$ at $V_{0}=V_{0}^{c}$.

According to (5.19), we have $\nu_{j}=L^{-2 j} \mu_{j}$, so Proposition 7.1 implies $\nu_{N}=O\left(\chi_{N} \bar{g}_{N} L^{-2 N}\right) \rightarrow 0$. We prove $\hat{\chi}=m^{-2}$ by showing that the $W$ and $K$ terms in (8.50) are even smaller than this, in fact by factors $O\left(\chi_{N} \bar{g}_{N}\right)$ and $O\left(\chi_{N} \bar{g}_{N}^{2}\right)$, respectively. For $\hat{\chi}^{\prime}$, by (8.47), the leading term $-m^{-4} \nu_{N}^{\prime}$ obeys the asymptotic formula desired for $\hat{\chi}^{\prime}$. Thus it suffices to show that $\nu_{0}$-derivatives of the $W$ and $K$ terms in (8.50) are relatively smaller as $m^{2} \downarrow 0$.

Recall the definitions of the $\mathcal{V}, \Phi_{N}$, and $T_{0, N}$ norms in (5.20), (6.10), and (6.13). The bounds for $W$ are more elementary than those for $K_{N}$, and are developed in [23]. By definition, $W_{j}(V, \tilde{V})$ is bilinear in $(V, \tilde{V})$, and by [23, (4.57)],

$$
\left\|W_{N}(V(\Lambda), \tilde{V}(\Lambda))\right\|_{T_{0, N}} \leq O\left(\chi_{N}\right)\|V\|_{\mathcal{V}}\|\tilde{V}\|_{\mathcal{V}}
$$

We write $W_{N}=W_{N}\left(V_{N}(\Lambda), V_{N}(\Lambda)\right)$ and $W_{N}^{\prime}=\frac{\partial}{\partial \nu_{0}} W_{N}$. Differentiation gives

$$
W_{N}^{\prime}=W_{N}\left(\Lambda ; V_{N}, V_{N}^{\prime}\right)+W_{N}\left(\Lambda ; V_{N}^{\prime}, V_{N}\right)
$$


and it then follows from (8.48) that

$$
\left\|W_{N}\right\|_{T_{0, N}} \leq O\left(\chi_{N} g_{N}^{2}\right), \quad\left\|W_{N}^{\prime}\right\|_{T_{0, N}} \leq O\left(\chi_{N} g_{N} \mu_{N}^{\prime}\right)
$$

By definition of the $T_{\phi}$ norm (see (6.13), or [21] for full details), for a differential form $F \in \mathcal{N}(\Lambda)$ with degree zero part $F^{0}$,

$$
\left|D^{2} F^{0}(0,0 ; f, f)\right| \leq 2\|F\|_{T_{0, N}}\|f\|_{\Phi_{N}}^{2} .
$$

The norm of the constant test function $1 \in \Phi_{N}$ is

$$
\|1\|_{\Phi_{N}}=\ell_{N}^{-1} \sup _{x}\left|1_{x}\right|=\ell_{N}^{-1}=O\left(L^{N[\phi]}\right),
$$

where $\ell_{N}=\ell_{0} L^{-N[\phi]}=\ell_{0} L^{-N(d-2) / 2}$. Since $|\Lambda|=L^{d N}$, the bound on $W_{N}$ of (8.53) therefore gives

$$
|\Lambda|^{-1}\left|D^{2} W_{N}^{0}(\Lambda ; 0,0 ; 1,1)\right| \leq 2|\Lambda|^{-1}\left\|W_{N}\right\|_{T_{0, N}}\|1\|_{\Phi_{N}}^{2} \leq O\left(\chi_{N} \bar{g}_{N}^{2} L^{-2 N}\right),
$$

and the right-hand side vanishes in the limit $N \rightarrow \infty$. Similarly, the $\nu_{0}$-derivative is bounded by $O\left(\chi_{N} \bar{g}_{N} L^{-2 N} \mu_{N}^{\prime}\right)=O\left(\chi_{N} \bar{g}_{N} \nu_{N}^{\prime}\right)$, so it is smaller than the leading contribution $\nu_{N}^{\prime}$ by a factor $O\left(\chi_{N} \bar{g}_{N}\right) \rightarrow 0$.

Proposition 8.1 and Lemma 8.6 provide bounds on $K_{N}$ and $K_{N}^{\prime}$ analogous to (8.53) with one more power of $g_{N}$, namely

$$
\left\|K_{N}\right\|_{T_{0, N}} \leq O\left(\chi_{N} g_{N}^{3}\right), \quad\left\|K_{N}^{\prime}\right\|_{T_{0, N}} \leq O\left(\chi_{N} g_{N}^{2} \mu_{N}^{\prime}\right)
$$

Thus the contribution due to $K$ is also small relative to the contributions due to $\nu$, and the proof is complete.

\subsection{Proof of Theorem $\mathbf{1 . 2}$}

Proof of Theorem 1.2. Let $g_{0}=\tilde{g}_{0}(g, 0)$, with $\tilde{g}_{0}$ the function of Proposition 4.2(ii). Let $\nu_{0}^{c}, z_{0}^{c}$ be given by $\mu_{0}^{c}, z_{0}^{c}$ of Proposition 7.1. By (4.4),

$$
\nu_{c}(g)=\frac{\nu_{0}^{c}\left(0, g_{0}\right)}{1+z_{0}^{c}\left(0, g_{0}\right)}=\nu_{0}^{c}\left(0, g_{0}\right)+O\left(g_{0}^{2}\right) .
$$

Since $g_{0}=g+O\left(g^{2}\right)$ by Proposition 4.2, it suffices to show that $\mu_{0}^{c}\left(0, g_{0}\right)=-2 C(0) g_{0}+O\left(g_{0}^{2}\right)=$ $-\mathrm{a} g_{0}+O\left(g_{0}^{2}\right)$ (all covariances have $m^{2}=0$ in this proof). By Proposition 7.1, $\mu_{0}^{c}=\check{\mu}_{0}$ where (see (6.4) and (8.6) ) the sequence $\check{\mu}_{j}$ satisfies

$$
\check{\mu}_{j+1}=L^{2} \check{\mu}_{j}\left(1-\gamma \beta_{j} \check{g}_{j}\right)+\eta_{j} \check{g}_{j}+O\left(\chi_{j} \bar{g}_{j}^{2}\right)
$$

and where we have used $\check{g}_{j}, \check{z}_{j}, \check{\mu}_{j}=O\left(\bar{g}_{j}\right)$ for the higher order terms, but not for the linear $\check{g}, \check{\mu}$ terms and not for the $\check{\mu} \check{g}$ term. We also recall from [6, (3.24), (4.4)] that

$$
\eta_{j}=2 L^{2(j+1)} C_{j+1 ; 0,0} .
$$


By Proposition 7.1, the sequence $\check{\mu}_{j}$ is bounded, so infinite iteration of (8.59) gives

$$
\check{\mu}_{0}=-\sum_{l=0}^{\infty}\left(L^{-2(l+1)} \prod_{k=0}^{l}\left(1-\gamma \beta_{k} \check{g}_{k}\right)^{-1}\right)\left(\eta_{l} \check{g}_{l}+O\left(\chi_{l} \bar{g}_{l}^{2}\right)\right) .
$$

By (8.28), we obtain from (8.60)- (8.61) that

$$
\check{\mu}_{0}=-2\left(1+O\left(g_{0}\right)\right) g_{0}^{\gamma} \sum_{l=0}^{\infty}\left(C_{l+1 ; 0,0} \check{g}_{l}^{1-\gamma}+O\left(L^{-2 l} \chi_{l} \bar{g}_{l}^{2-\gamma}\right)\right) .
$$

Since $C(0)=\sum_{l=0}^{\infty} C_{l+1 ; 0,0}$, this gives

$$
\begin{gathered}
\check{\mu}_{0}=-2 C(0) g_{0}\left(1+O\left(g_{0}\right)\right)-2\left(1+O\left(g_{0}\right)\right) g_{0}^{\gamma} \sum_{l=0}^{\infty} C_{l+1 ; 0,0}\left(\check{g}_{l}^{1-\gamma}-g_{0}^{1-\gamma}\right) \\
+g_{0}^{\gamma} \sum_{l=0}^{\infty} O\left(L^{-2 l} \bar{g}_{l}^{2-\gamma}\right) .
\end{gathered}
$$

We show that the last two terms are $O\left(g_{0}^{2}\right)$. By (8.21) with $\psi(t)=(1-\gamma)^{-1} t^{-\gamma}$,

$$
\check{g}_{l}^{1-\gamma}-g_{0}^{1-\gamma}=\int_{\check{g}_{0}}^{\check{g}_{l}} \psi(t) d t=(1-\gamma)^{-1} \sum_{k=0}^{l-1} \beta_{k} \check{g}_{k}^{2-\gamma}+O\left(g_{0}^{2-\gamma}\right) .
$$

Thus, by Fubini's theorem and $C_{l+1 ; 0,0}=O\left(L^{-2 l}\right)$, the middle sum in (8.63) is bounded by a multiple of

$$
\begin{aligned}
g_{0}^{\gamma} \sum_{l=0}^{\infty} C_{l+1 ; 0,0}\left(\check{g}_{l}^{1-\gamma}-\check{g}_{0}^{1-\gamma}\right) & =(1-\gamma)^{-1} g_{0}^{\gamma} \sum_{k=0}^{\infty} \beta_{k} \check{g}_{k}^{2-\gamma} \sum_{l=k+1}^{\infty} C_{l+1 ; 0,0}+O\left(g_{0}^{2}\right) \\
& =(1-\gamma)^{-1} g_{0}^{\gamma} \sum_{k=0}^{\infty} \beta_{k} \check{g}_{k}^{2-\gamma} O\left(L^{-2 k}\right)+O\left(g_{0}^{2}\right)=O\left(g_{0}^{2}\right)
\end{aligned}
$$

Similarly, the rightmost sum in (8.63) is bounded by $O\left(g_{0}^{2}\right)$, so

$$
\check{\mu}_{0}=-2 C(0) g_{0}+O\left(g_{0}^{2}\right),
$$

and the proof is complete.

\section{A Existence of critical value}

The following lemma proves existence of a critical value in $(-\infty, 0]$. For dimensions $d>2$, a simple proof rules out $-\infty$. Although our main results pertain only to $d \geq 4$, we nevertheless show that the critical value is finite in all dimensions. 
Lemma A.1. For all dimensions $d>0$, there exists a critical value $\nu_{c}=\nu_{c}(d, g) \in(-\infty, 0]$ such that $\chi(g, \nu)<\infty$ if and only if $\nu>\nu_{c}$. For $d>2, \nu_{c} \in\left[-2 C_{0}(0) g, 0\right]$, where $C_{0}(x)=\left(-\Delta_{\mathbb{Z}^{d}}^{-1}\right)_{0, x}$ is the Green function of the simple random walk.

Proof. We first show that $c_{T+S} \leq c_{T} c_{S}$ for all $S, T \geq 0$. To prove this, let

$$
I(S, T)=\int_{S}^{T} \int_{S}^{T} \mathbb{1}_{X_{S_{1}}=X_{S_{2}}} d S_{1} d S_{2} .
$$

Then

$$
I(T)=I(0, T) \geq I(0, S)+I(S, T) .
$$

By the Markov property, $I(0, T)$ and $I(T, T+S)$ are conditionally independent given $X(T)$. Using translation-invariance, it therefore follows that

$$
c_{T+S} \leq E\left(e^{-g I(0, T)} e^{-g I(T, T+S)}\right)=E\left(e^{-g I(0, T)}\right) E\left(e^{-g I(T, T+S)}\right)=c_{T} c_{S} .
$$

A standard lemma for subadditive functions now yields the existence of a critical value $\nu_{c}=\nu_{c}(g) \in$ $[-\infty, \infty)$ such that $c_{T}^{1 / T} \rightarrow e^{\nu_{c}}$ and also $c_{T} \geq e^{\nu_{c} T}$ (see, e.g., [51, Lemma 1.2.2]). The inequality $\nu_{c} \leq 0$ is obvious from $I(S, T) \geq 0$. For $d>2$, we show that $\nu_{c} \in\left[-2 C_{0}(0) g, 0\right]$, as follows. By Jensen's inequality,

$$
c_{T}=E\left(e^{-g I(T)}\right) \geq e^{-g E(I(T))} .
$$

An elementary estimate shows that $E(I(T)) \leq 2 T C_{0}(0)$, and the Green function $C_{0}(x)$ is finite for $d>2$ (the estimate can be done as in the discrete case, see, e.g., [25, Lemma 1.4]). This shows that $\nu_{c} \in\left[-2 C_{0}(0) g, 0\right]$.

To prove that $\nu_{c}>-\infty$ also in lower dimensions, we proceed as follows. Let $S_{T, n}$ be the event that the path followed by $X[0, T]$ is an $n$-step strictly self-avoiding walk. Then

$$
\chi(\nu)=\int_{0}^{\infty} E\left(e^{-g I(T)}\right) e^{-\nu T} d T \geq \sum_{n=0}^{\infty} \int_{0}^{\infty} E\left(e^{-g I(T)} \mid S_{T, n}\right) P\left(S_{T, n}\right) e^{-\nu T} d T .
$$

Let $s_{n}$ denote the number of $n$-step (discrete-time) strictly self-avoiding walks that start at the origin. The connective constant $\mu=\lim _{n \rightarrow \infty} s_{n}^{1 / n}$ exists and lies in $[d, 2 d-1]$. Let $Y_{T}$ denote the number of steps taken by $X$ during the interval $[0, T]$; this is a Poisson random variable with mean $2 d T$. For any $\mu^{\prime}<\mu$ there is a constant $k$ such that

$$
P\left(S_{T, n}\right)=\frac{s_{n}}{(2 d)^{n}} P\left(Y_{T}=n\right)=\frac{s_{n}}{(2 d)^{n}} \frac{e^{-2 d T}(2 d T)^{n}}{n !} \geq k \frac{e^{-2 d T}\left(\mu^{\prime} T\right)^{n}}{n !} .
$$

Also, by Jensen's inequality,

$$
E\left(e^{-g I(T)} \mid S_{T, n}\right) \geq e^{-g E\left(I(T) \mid S_{T, n}\right)} .
$$

To evaluate $E\left(I(T) \mid S_{T, n}\right)$, we first observe that on the event $S_{T, n}$ the $n$ jump times of the walk are independent and uniformly distributed on $[0, T]$, and in particular the lengths of the $n+1$ subintervals of $[0, T]$ determined by the jump times are identically distributed. Let $U_{0}=0$, 
$U_{n+1}=T$, and let $U_{1}, \ldots, U_{n}$ be independent uniform random variables on $[0, T]$ with order statistics $U_{(1)}, \ldots, U_{(n)}$ (i.e., ordered from smaller to larger values). Then

$$
E\left(I(T) \mid S_{T, n}\right)=E\left[\sum_{i=1}^{n+1}\left(U_{(i)}-U_{(i-1)}\right)^{2}\right]=(n+1) E U_{(1)}^{2} .
$$

The probability density function of $U_{(1)}$ is $f(x)=n T^{-n}(T-x)^{n-1}$, so

$$
E\left(I(T) \mid S_{T, n}\right)=\frac{(n+1) n}{T^{n}} \int_{0}^{T} x^{2}(T-x)^{n-1} d x=\frac{2 T^{2}}{(n+2)} .
$$

By (A.7) and (A.9),

$$
E\left(e^{-g I(T)} \mid S_{T, n}\right) \geq e^{-2 g T^{2} /(n+2)} \geq e^{-2 g T^{2} / n} .
$$

Therefore, by (A.5) - A.6 and with $h=2 g$,

$$
\begin{aligned}
\chi(\nu) & \geq k \sum_{n=1}^{\infty} \int_{0}^{\infty} e^{-h T^{2} / n} \frac{e^{-2 d T}\left(\mu^{\prime} T\right)^{n}}{n !} e^{-\nu T} d T \\
& =k \sum_{n=1}^{\infty} \int_{0}^{\infty} e^{-h n T^{2}} \frac{e^{-2 d n T}\left(\mu^{\prime} n T\right)^{n}}{n !} e^{-\nu n T} n d T .
\end{aligned}
$$

Using Stirling's formula and $\mu^{\prime} T \geq 1$ in the restricted integration domain, we obtain

$$
\chi(\nu) \geq k \sum_{n=1}^{\infty} \frac{n e^{n}}{\sqrt{2 \pi n}} \int_{\left(\mu^{\prime}\right)^{-1}}^{\infty} e^{-n\left(h T^{2}+(2 d+\nu) T\right)} d T .
$$

We consider $\nu<-2 d$ and complete the square to get

$$
h T^{2}+(2 d+\nu) T=h\left(T-c_{\nu}\right)^{2}-h c_{\nu}^{2}, \quad \text { with } c_{\nu}=(|\nu|-2 d) /(2 h) .
$$

We further assume that $c_{\nu}>\left(\mu^{\prime}\right)^{-1}$, i.e., that $|\nu|>2 d+2 h\left(\mu^{\prime}\right)^{-1}$, and use Laplace's method to get, as $n \rightarrow \infty$,

$$
\int_{\left(\mu^{\prime}\right)^{-1}}^{\infty} e^{-n\left(h T^{2}+(2 d+\nu) T\right)} d T \sim \operatorname{const} \frac{e^{n h c_{\nu}^{2}}}{\sqrt{n}} .
$$

With (A.12), this gives a divergent lower bound on the susceptibility, so $\nu_{c} \in\left[-2 d-2 h\left(\mu^{\prime}\right)^{-1}, 0\right]$ and the proof is complete.

Concerning explicit bounds on the critical value for $d=1,2$, since $\mu^{\prime}$ is an arbitrary number less than $\mu$, the above proof gives $\nu_{c} \in\left[-2 d-4 g \mu^{-1}, 0\right]$. Using $\mu=1$ for $d=1$, and $\mu^{-1} \leq \frac{1}{2}$ for $d=2$, this gives $\nu_{c} \in[-2-4 g, 0]$ for $d=1$ and $\nu_{c} \in[-4-2 g, 0]$ for $d=2$. 


\section{Acknowledgements}

This work was supported in part by NSERC of Canada. This material is also based upon work supported by the National Science Foundation under agreement No. DMS-1128155. RB gratefully acknowledges the support of the University of British Columbia, where he was a PhD student while much of his work was done. Part of this work was done away from the authors' home institutions, and we gratefully acknowledge the support and hospitality of the IAM at the University of Bonn and the Department of Mathematics and Statistics at McGill University (RB), the Institute for Advanced Study at Princeton and Eurandom (DB), and the Institut Henri Poincaré and the Mathematical Institute of Leiden University (GS). We thank Alexandre Tomberg for useful discussions, and an anonymous referee for helpful comments.

\section{References}

[1] M. Aizenman. Geometric analysis of $\varphi^{4}$ fields and Ising models, Parts I and II. Commun. Math. Phys., 86:1-48, (1982).

[2] C. Aragão de Carvalho, S. Caracciolo, and J. Fröhlich. Polymers and $g|\phi|^{4}$ theory in four dimensions. Nucl. Phys. B, 215 [FS7]:209-248, (1983).

[3] R. Bauerschmidt. A simple method for finite range decomposition of quadratic forms and Gaussian fields. Probab. Theory Related Fields, 157:817-845, (2013).

[4] R. Bauerschmidt, D.C. Brydges, and G. Slade. In preparation.

[5] R. Bauerschmidt, D.C. Brydges, and G. Slade. Critical two-point function of the 4-dimensional weakly self-avoiding walk. To appear in Commun. Math. Phys.

[6] R. Bauerschmidt, D.C. Brydges, and G. Slade. A renormalisation group method. III. Perturbative analysis. To appear in J. Stat. Phys.

[7] R. Bauerschmidt, D.C. Brydges, and G. Slade. Structural stability of a dynamical system near a non-hyperbolic fixed point. Annales Henri Poincaré, doi:10.1007/s00023-014-0338-0.

[8] R. Bauerschmidt, D.C. Brydges, and G. Slade. Scaling limits and critical behaviour of the 4-dimensional $n$-component $|\varphi|^{4}$ spin model. J. Stat. Phys, 157:692-742, (2014).

[9] R. Bauerschmidt, H. Duminil-Copin, J. Goodman, and G. Slade. Lectures on self-avoiding walks. In D. Ellwood, C. Newman, V. Sidoravicius, and W. Werner, editors, Probability and Statistical Physics in Two and More Dimensions, pages 395-467. Clay Mathematics Proceedings, vol. 15, Amer. Math. Soc., Providence, RI, (2012).

[10] F.A. Berezin. The Method of Second Quantization. Academic Press, New York, (1966).

[11] A. Bovier, G. Felder, and J. Fröhlich. On the critical properties of the Edwards and the self-avoiding walk model of polymer chains. Nucl. Phys. B, 230 [FS10]:119-147, (1984). 
[12] E. Brézin, J.C. Le Guillou, and J. Zinn-Justin. Approach to scaling in renormalized perturbation theory. Phys. Rev. D, 8:2418-2430, (1973).

[13] D. Brydges, S.N. Evans, and J.Z. Imbrie. Self-avoiding walk on a hierarchical lattice in four dimensions. Ann. Probab., 20:82-124, (1992).

[14] D. Brydges and G. Slade. Renormalisation group analysis of weakly self-avoiding walk in dimensions four and higher. In R. Bhatia et al, editor, Proceedings of the International Congress of Mathematicians, Hyderabad 2010, pages 2232-2257, Singapore, (2011). World Scientific.

[15] D.C. Brydges. Lectures on the renormalisation group. In S. Sheffield and T. Spencer, editors, Statistical Mechanics, pages 7-93. American Mathematical Society, Providence, (2009). IAS/Park City Mathematics Series, Volume 16.

[16] D.C. Brydges, A. Dahlqvist, and G. Slade. The strong interaction limit of continuous-time weakly self-avoiding walk. In J.-D. Deuschel, B. Gentz, W. König, M. von Renesse, M. Scheutzow, and U. Schmock, editors, Probability in Complex Physical Systems: In Honour of Erwin Bolthausen and Jürgen Gärtner, Springer Proceedings in Mathematics, Volume 11, pages 275-287, Berlin, (2012). Springer.

[17] D.C. Brydges, G. Guadagni, and P.K. Mitter. Finite range decomposition of Gaussian processes. J. Stat. Phys., 115:415-449, (2004).

[18] D.C. Brydges and J.Z. Imbrie. End-to-end distance from the Green's function for a hierarchical self-avoiding walk in four dimensions. Commun. Math. Phys., 239:523-547, (2003).

[19] D.C. Brydges and J.Z. Imbrie. Green's function for a hierarchical self-avoiding walk in four dimensions. Commun. Math. Phys., 239:549-584, (2003).

[20] D.C. Brydges, J.Z. Imbrie, and G. Slade. Functional integral representations for self-avoiding walk. Probab. Surveys, 6:34-61, (2009).

[21] D.C. Brydges and G. Slade. A renormalisation group method. I. Gaussian integration and normed algebras. To appear in J. Stat. Phys.

[22] D.C. Brydges and G. Slade. A renormalisation group method. II. Approximation by local polynomials. To appear in J. Stat. Phys.

[23] D.C. Brydges and G. Slade. A renormalisation group method. IV. Stability analysis. To appear in J. Stat. Phys.

[24] D.C. Brydges and G. Slade. A renormalisation group method. V. A single renormalisation group step. To appear in J. Stat. Phys.

[25] D.C. Brydges and G. Slade. The diffusive phase of a model of self-interacting walks. Probab. Theory Related Fields, 103:285-315, (1995).

[26] D.C. Brydges and T. Spencer. Self-avoiding walk in 5 or more dimensions. Commun. Math. Phys., 97:125-148, (1985). 
[27] M. Disertori and T. Spencer. Anderson localization for a supersymmetric sigma model. Commun. Math. Phys., 300:659-671, (2010).

[28] M. Disertori, T. Spencer, and M.R. Zirnbauer. Quasi-diffusion in a 3D supersymmetric hyperbolic sigma model. Commun. Math. Phys., 300:435-486, (2010).

[29] B. Duplantier. Polymer chains in four dimensions. Nucl. Phys. B, 275 [FS17]:319-355, (1986).

[30] J. Feldman, J. Magnen, V. Rivasseau, and R. Sénéor. Construction and Borel summability of infrared $\Phi_{4}^{4}$ by a phase space expansion. Commun. Math. Phys., 109:437-480, (1987).

[31] W. Feller. An Introduction to Probability Theory and Its Applications, Volume II. Wiley, New York, 2nd edition, (1971).

[32] J. Fröhlich. On the triviality of $\varphi_{d}^{4}$ theories and the approach to the critical point in $d \geq 4$ dimensions. Nucl. Phys., B200 [FS4]:281-296, (1982).

[33] K. Gawȩdzki and A. Kupiainen. Massless lattice $\varphi_{4}^{4}$ theory: Rigorous control of a renormalizable asymptotically free model. Commun. Math. Phys., 99:199-252, (1985).

[34] K. Gawȩdzki and A. Kupiainen. Asymptotic freedom beyond perturbation theory. In K. Osterwalder and R. Stora, editors, Critical Phenomena, Random Systems, Gauge Theories, Amsterdam, (1986). North-Holland. Les Houches 1984.

[35] P.G. de Gennes. Exponents for the excluded volume problem as derived by the Wilson method. Phys. Lett., A38:339-340, (1972).

[36] S.E. Golowich and J.Z. Imbrie. The broken supersymmetry phase of a self-avoiding random walk. Commun. Math. Phys., 168:265-319, (1995).

[37] C. Gruber and H. Kunz. General properties of polymer systems. Commun. Math. Phys., 22:133-161, (1971).

[38] T. Hara. A rigorous control of logarithmic corrections in four dimensional $\varphi^{4}$ spin systems. I. Trajectory of effective Hamiltonians. J. Stat. Phys., 47:57-98, (1987).

[39] T. Hara. Decay of correlations in nearest-neighbor self-avoiding walk, percolation, lattice trees and animals. Ann. Probab., 36:530-593, (2008).

[40] T. Hara and G. Slade. Self-avoiding walk in five or more dimensions. I. The critical behaviour. Commun. Math. Phys., 147:101-136, (1992).

[41] T. Hara and H. Tasaki. A rigorous control of logarithmic corrections in four dimensional $\varphi^{4}$ spin systems. II. Critical behaviour of susceptibility and correlation length. J. Stat. Phys., 47:99-121, (1987).

[42] F. den Hollander. Random Polymers. Springer, Berlin, (2009). Lecture Notes in Mathematics Vol. 1974. Ecole d'Eté de Probabilités de Saint-Flour XXXVII-2007. 
[43] D. Iagolnitzer and J. Magnen. Polymers in a weak random potential in dimension four: rigorous renormalization group analysis. Commun. Math. Phys., 162:85-121, (1994).

[44] C. Itzykson and J-M. Drouffe. Statistical Field Theory, volume I. Cambridge University Press, Cambridge, (1989).

[45] A.I. Larkin and D.E. Khmel'Nitskii. Phase transition in uniaxial ferroelectrics. Soviet Physics JETP, 29:1123-1128, (1969). English translation of Zh. Eksp. Teor. Fiz. 56, 2087-2098, (1969).

[46] G.F. Lawler. Gaussian behavior of loop-erased self-avoiding random walk in four dimensions. Duke Math. J., 53:249-269, (1986).

[47] G.F. Lawler. The logarithmic correction for loop-erased walk in four dimensions. Journal of Fourier Analysis and Applications, Special Issue: Proceedings of the Conference in Honor of Jean-Pierre Kahane (Orsay, June 28 - July 3 1993):347-362, (1995).

[48] G.F. Lawler, O. Schramm, and W. Werner. On the scaling limit of planar self-avoiding walk. Proc. Symposia Pure Math., 72:339-364, (2004).

[49] Y. Le Jan. Temps local et superchamp. In Séminaire de Probabilités XXI. Lecture Notes in Mathematics \#1247, pages 176-190, Berlin, (1987). Springer.

[50] J.M. Luttinger. The asymptotic evaluation of a class of path integrals. II. J. Math. Phys., 24:2070-2073, (1983).

[51] N. Madras and G. Slade. The Self-Avoiding Walk. Birkhäuser, Boston, (1993).

[52] A.J. McKane. Reformulation of $n \rightarrow 0$ models using anticommuting scalar fields. Phys. Lett. A, 76:22-24, (1980).

[53] P.K. Mitter and B. Scoppola. The global renormalization group trajectory in a critical supersymmetric field theory on the lattice $\mathbf{Z}^{3}$. J. Stat. Phys., 133:921-1011, (2008).

[54] B. Nienhuis. Exact critical exponents of the $O(n)$ models in two dimensions. Phys. Rev. Lett., 49:1062-1065, (1982).

[55] M. Ohno. In preparation.

[56] G. Parisi and N. Sourlas. Self-avoiding walk and supersymmetry. J. Phys. Lett., 41:L403L406, (1980).

[57] W. Rudin. Principles of Mathematical Analysis. McGraw-Hill, New York, 3rd edition, (1976).

[58] R.D. Schram, G.T. Barkema, and R.H. Bisseling. Exact enumeration of self-avoiding walks. J. Stat. Mech., P06019, (2011).

[59] G. Slade. The Lace Expansion and its Applications. Springer, Berlin, (2006). Lecture Notes in Mathematics Vol. 1879. Ecole d'Eté de Probabilités de Saint-Flour XXXIV-2004. 
[60] G. Slade and A. Tomberg. Critical correlation functions for the 4-dimensional weakly selfavoiding walk and $n$-component $|\varphi|^{4}$ model. Preprint, (2014).

[61] F.J. Wegner and E.K. Riedel. Logarithmic corrections to the molecular-field behavior of critical and tricritical systems. Phys. Rev. B, 7:248-256, (1973).

[62] K.G. Wilson and J. Kogut. The renormalization group and the $\epsilon$ expansion. Phys. Rep., 12:75-200, (1974). 